\title{
High p16 expression and heterozygous RB1 loss are biomarkers for CDK4/6 inhibitor resistance in ER+ breast cancer
}

\section{Marta Palafox}

Vall d'Hebron Institue of Oncology

Laia Monserrat

Vall d'Hebron Institue of Oncology

Meritxell Bellet

Vall d'Hebron Institute of Oncology, Barcelona, Spain.

Guillermo Villacampa

Vall d'Hebron Institue of Oncology

Abel Gonzalez-Perez

Institute for Research in Biomedicine https://orcid.org/0000-0002-8582-4660

Mafalda Oliveira

Vall d'Hebron Institute of Oncology, Barcelona, Spain. https://orcid.org/0000-0001-9152-8799

Fara Brasó-Maristany

Consorci Institut D'Investigacions Biomediques August Pi I Sunyer

Nusaibah Ibrahimi

Gustave Roussy, Université Paris-Saclay https://orcid.org/0000-0003-4537-0323

Srinivasaraghavan Kannan

Bioinformatics Institute https://orcid.org/0000-0002-9539-5249

Leonardo Mina

MedSir

María Teresa Herrera-Abreu

Institute of Cancer Research

Andreu Odena

Vall d'Hebron Institute of Oncology

Mónica Sánchez-Guixé

Institute for Research in Biomedicine https://orcid.org/0000-0002-9430-4413

Marta Capelán

Vall d’Hebron Institute of Oncology

Analía Azaro

Vall d’Hebron Institute of Oncology

Alejandra Bruna 
The Institute of cancer Research

\section{Olga Rodriguez}

Vall d'Hebron Institute of Oncology

\section{Marta Guzmán}

Vall d'Hebron Institute of Oncology

Judit Grueso

\section{Cristina Viaplana}

Vall d'Hebron Institute of Oncology

Javier Hernandez-Losa

Hospital Universitario Vall d’Hebron https://orcid.org/0000-0003-1526-3201

\section{Faye Su}

Novartis

\section{Kui Lin}

Genentech, Inc.

\section{Robert (B.) Clarke}

University of Manchester https://orcid.org/0000-0001-5407-3123

\section{Carlos Caldas}

Cancer Research UK Cambridge Institute, University of Cambridge, Cambridge https://orcid.org/00000003-3547-1489

\section{Arribas Joaquín}

Vall d'Hebron Institute of Oncology https://orcid.org/0000-0002-0504-0664

\section{Stefan Michiels}

Gustave Roussy, CESP, U1018, Université Paris-Sud, Faculté de Médcine, Université Paris-Saclay https://orcid.org/0000-0002-6963-2968

\section{Alicia García-Sanz}

MedSir

\section{Nicholas C. Turner}

Institute of Cancer Research

\section{Aleix Prat}

Medical Oncology Hospital Clínic, Barcelona; Translational Genomics and Targeted Therapeutics Group, IDIBAPS https://orcid.org/0000-0003-2377-540X

\section{Paolo Nuciforo}

Vall d'Hebron Institute of Oncology https://orcid.org/0000-0003-1380-0990

\section{Rodgrigo Dienstmann}

Vall d'Hebron

\section{Chandra Verma}

A*STAR

\section{Núria López-Bigas}

Institute for Research in Biomedicine (IRB Barcelona) 


\section{Maurizio Scaltriti}

Astra-Zeneca https://orcid.org/0000-0002-5522-1447

Monica Arnedos

departement of medical oncology, Gustave Roussy, Université Paris Saclay

Cristina Saura

VHIO https://orcid.org/0000-0001-8296-5065

Violeta Serra ( $\nabla$ vserra@vhio.net)

VHIO https://orcid.org/0000-0001-6620-1065

\section{Article}

Keywords: ER+ breast cancer, CDK4/ 6 inhibitors, patient-derived xenoimplants (PDX)

Posted Date: March 10th, 2021

DOI: https://doi.org/10.21203/rs.3.rs-236645/v1

License: (c) (1) This work is licensed under a Creative Commons Attribution 4.0 International License.

Read Full License

Version of Record: A version of this preprint was published at Nature Communications on September 7th, 2022. See the published version at https://doi.org/10.1038/s41467-022-32828-6. 


\section{High p16 expression and heterozygous RB1 loss are biomarkers for 2 CDK4/6 inhibitor resistance in $\mathrm{ER}^{+}$breast cancer}

3 Marta Palafox ${ }^{1}$, Laia Monserrat ${ }^{1}$, Meritxell Bellet ${ }^{2,3}$, Guillermo Villacampa ${ }^{4}$, Abel Gonzalez-

4 Perez $^{5,6}$, Mafalda Oliveira ${ }^{2,3}$, Fara Brasó-Maristany ${ }^{7}$, Nusaibah Ibrahimi ${ }^{8,9}$, Srinivasaraghavan 5 Kannan $^{10}$, Leonardo Mina ${ }^{11}$, Maria Teresa Herrera-Abreu ${ }^{12}$, Andreu Ódena ${ }^{1}$, Mónica Sánchez-

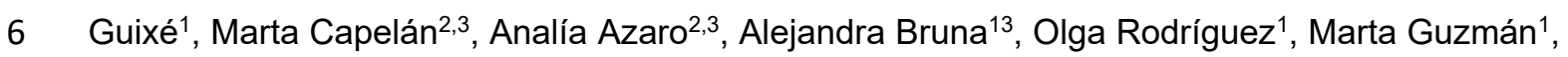
7 Judit Grueso ${ }^{1}$, Cristina Viaplana ${ }^{4}$, Javier Hernández ${ }^{14}$, Faye Su ${ }^{15}$, Kui Lin ${ }^{16}$, Robert B. Clarke ${ }^{17}$, 8 Carlos Caldas $^{18}$, Joaquín Arribas ${ }^{19,20,21,22,23}$, Stefan Michiels ${ }^{8,9}$, Alicia García-Sanz ${ }^{11}$, Nicholas C. 9 Turner $^{12}$, Aleix Prat ${ }^{7,24,25,26,27}$, Paolo Nuciforo ${ }^{28}$, Rodrigo Dienstmann ${ }^{4}$, Chandra Verma ${ }^{10,29,30}$, 10 Nuria Lopez-Bigas ${ }^{5,6,31}$, Maurizio Scaltriti ${ }^{32}$, Monica Arnedos $^{33,34}$, Cristina Saura ${ }^{2,3}$, Violeta 11 Serra $^{1,19^{*}}$

${ }^{1}$ Experimental Therapeutics Group, Vall d'Hebron Institute of Oncology, Barcelona, Spain

${ }^{2}$ Breast Cancer and Melanoma Group, Vall d'Hebron Institute of Oncology, Barcelona, Spain

${ }^{3}$ Department of Medical Oncology, Hospital Vall d'Hebron, Universitat Autònoma de Barcelona,

Barcelona, Spain

${ }^{4}$ Oncology Data Science Group, Vall d'Hebron Institute of Oncology, Vall d'Hebron University

Campus, Barcelona, Spain

5Institute for Research in Biomedicine (IRB Barcelona), The Barcelona Institute of Science and

Technology, Barcelona, Spain

${ }^{6}$ Research Program on Biomedical Informatics, Universitat Pompeu Fabra, Barcelona, Spain.

${ }^{7}$ Translational Genomics and Targeted Therapies in Solid Tumors, August Pi i Sunyer

Biomedical Research Institute (IDIBAPS), Barcelona, Spain

${ }^{8}$ Service de Biostatistique et d'Epidémiologie, Gustave Roussy, Université Paris-Saclay, Villejuif, France.

${ }^{9}$ Oncostat U1018, Inserm, University Paris-Saclay, labeled Ligue Contre le Cancer, Villejuif, France.

${ }^{10}$ Bioinformatics Institute, \#07-01 Matrix, Singapore

${ }^{11}$ Medica Scientia Innovation Research (MedSIR), Barcelona, Spain 
${ }^{12}$ The Breast Cancer Now Research Centre, Institute of Cancer Research, London, UK

${ }^{13}$ Preclinical Modelling of Pediatric Cancer Evolution Group, Centre for Evolution and Cancer, The Institute of Cancer Research, London, UK

${ }^{14}$ Translational Molecular Pathology, Vall d'Hebron Institute of Research (VHIR), Universitat Autònoma de Barcelona, Barcelona, Spain

${ }^{15}$ Novartis Pharmaceuticals, East Hanover, NJ, USA

${ }^{16}$ Genentech, Inc., South San Francisco, California, USA

${ }^{17}$ Breast Biology Group, Manchester Breast Centre, Division of Cancer Sciences, Faculty of Biology, Medicine and Health, University of Manchester, Manchester, UK

${ }^{18}$ Cancer Research UK, Cambridge Institute, Cambridge, UK

${ }^{19} \mathrm{CIBERONC}$, Vall d'Hebron Institute of Oncology, Barcelona, Spain

${ }^{20}$ Growth Factors Laboratory, Vall d'Hebron Institute of Oncology, Barcelona, Spain.

${ }^{21}$ Department of Biochemistry and Molecular Biology, Universitat Autònoma de Barcelona, Bellaterra, Barcelona, Spain.

${ }^{22}$ Cancer Research Program, IMIM (Hospital del Mar Medical Research Institute), Barcelona, Spain.

${ }^{23}$ Institució Catalana de Recerca i Estudis Avançats (ICREA), Barcelona, Spain

${ }^{24}$ University of Barcelona, Barcelona, Spain

${ }^{25}$ Department of Medical Oncology, Hospital Clinic, Barcelona, Spain

${ }^{26}$ SOLTI Breast Cancer Research Group, Barcelona, Spain.

${ }^{27}$ Department of Oncology, IOB Institute of Oncology, Quironsalud Group, Barcelona, Spain.

${ }^{28}$ Molecular Oncology Group, Vall d'Hebron Institute of Oncology, Barcelona, Spain

${ }^{29}$ School of Biological Sciences, Nanyang Technological University, Singapore

${ }^{30}$ Department of Biological Sciences, National University of Singapore, Singapore

${ }^{31}$ Institució Catalana de Recerca i Estudis Avançats, Barcelona, Spain

${ }^{32}$ Departments of Pathology and Human Oncology and Pathogenesis Program, Memorial SloanKettering Cancer Center, New York, USA

${ }^{33}$ Department of Medical Oncology, Gustave Roussy, Villejuif, France

${ }^{34}$ Inserm Unit U981, Villejuif, France 
${ }^{*}$ Corresponding author: Violeta Serra

Vall d'Hebron Institute of Oncology (VHIO), Barcelona, Spain. Carrer Natzaret 115-117

(CELLEX building), 08035 Barcelona, Spain. vserra@vhio.net; www.vhio.net; Phone: +34932543450

Running title: $\mathrm{p} 16$-high and $R B 1$ heterozygous loss in resistance to CDK4/6i

Keywords: $\mathrm{ER}^{+}$breast cancer, CDK4/6 inhibitors, patient-derived xenoimplants (PDX).

Financial support: We acknowledge Novartis, Genentech, the GHD-Pink program, the FERO Foundation, and the Orozco Family for supporting this study [to V.S.]. This study has also been supported by the Catalan Agency AGAUR (2017 SGR 540) [to V.S.]. V.S. received funds from the Instituto de Salud Carlos III: grants PI13/01714, CP14/00228, MV15/00041 and CPII19/00033. M.P. received a Juan de la Cierva Grant from the Ministerio de Economía y Competitividad (FJCI-2015-25412), L.M. a grant from FI-AGAUR (2019 FI_B 01199), F.B-M. a grant from the Fundación Científica Asociación Española Contra el Cáncer (AECC_Postdoctoral17-1062) and M.S-G, a Marie Slodowska-Curie Innovative Training Networks PhD fellowship (H2020-MSCA-ITN-2015_675392). This work was supported by Breast Cancer Research Foundation (BCRF-19-08), Instituto de Salud Carlos III Project Reference number AC15/00062 and the EC under the framework of the ERA-NET TRANSCAN-2 initiative co-financed by FEDER, Instituto de Salud Carlos III (CB16/12/00449 and PI19/01181), and Asociación Española Contra el Cáncer (to J.A.). R.B.C. laboratory is supported by Breast Cancer Now (grant numbers: MAN-Q1 and MAN-Q2), NIHR Manchester Biomedical Research Centre (IS-BRC-1215-20007) and EdiREX Horizon 2020 grant No.731105. The xenograft program in the C.C. laboratory is supported by Cancer Research UK and also received funding from an EU H2020 Network of Excellence (EuroCAN). This work has been supported by NIH grants P30 CA008748 and RO1CA190642-01, the CDMRP grant BC171535P1, and the Breast Cancer Research Foundation [to M.S.]. A.P. received funds from Instituto de Salud Carlos IIIPI16/00904 and PI19/01846, Breast Cancer Now-2018NOVPCC1294, Breast Cancer Research Foundation-AACR Career Development Awards for Translational Breast Cancer Research 1920-26-PRAT, Fundació La Marató TV3 201935-30, the European Union's Horizon 2020 research and innovation programme H2020-SC1-BHC-2018-2020. IRB Barcelona is a recipient of a Severo 
Ochoa Centre of Excellence Award from the Spanish Ministry of Economy and Competitiveness (MINECO; Government of Spain) and is supported by CERCA (Generalitat de Catalunya). ABCPOP received funding from the Breast Cancer Research Foundation and research grants from Pfizer and Eli-Lilly, respectively (to M.A.). C. S. Verma reports granst from MSD International and grants from Ipsen outside the submitted work.

Conflict of interest: V.S. received non-commercial research support from Novartis and Genentech. M.B. reported receiving honoraria for speaker activities and advisory role from Pfizer, Novartis and Lilly and support for travel expenses from Roche and Pfizer. M.O. declares grant/research support (to the Institution) from AstraZeneca, Philips Healthcare, Genentech, Roche, Novartis, Immunomedics, Seattle Genetics, GSK, Boehringer-Ingelheim, PUMA Biotechnology, and Zenith Epigenetics; consultant role for Roche, GSK, PUMA Biotechnology, AstraZeneca, and Seattle Genetics; and has received honoraria from Roche, Seattle Genetics, and Novartis. G.V. reported receiving honoraria for speaker activities from MDS and advisory role from Astrazeneca. F.S. is employee of Novartis. K.L. is employee of Genentech. C.C. is a member of AstraZeneca's External Science Panel, of Illumina's Scientific Advisory Board, and is a recipient of research grants (administered by the University of Cambridge) from AstraZeneca, Genentech, Roche, and Servier. J.A. has received research funds from Roche, Synthon, Menarini, and Molecular Partners and consultancy honoraria from Menarini. A.P. reports that his institution received research funding from Nanostring Technologies, Roche and Novartis and reports consulting and lecture fees from Nanostring Technologies, Roche, Novartis, Pfizer, Oncolytics Biotech, Amgen, Lilly, MSD and PUMA. P.N. has consulted for Bayer, Novartis, and MSD and received compensation. R.D. is on advisory role of AstraZeneca, Roche and Boehringer-Ingelheim and has received speaker's fees from Roche, Symphogen, IPSEN, Amgen, Servier, Sanofi, MSD; and research support from Merck. M.S. is on the scientific advisory board of Menarini Ricerche and the Bioscience Institute, has received research funds from Puma Biotechnology, Daiichi-Sankio, AstraZeneca, Targimmune, Immunomedics and Menarini Ricerche, and is a cofounder of Medendi.org. M.A. received a research grant from Eli-Lilly, honoraria from Novartis, Astrazeneca, Seattle Genetics, Abbvie and Pfizer and travel grants from Novartis, Roche, Pfizer. S.M. has provide punctual statistical advice to IDDI and Janssen Cilag 
and participated to data and safety monitoring committees of clinical trials (Hexal, Steba, IQVIA,

121 Roche, Sensorion, Biophytis, Servier, Yuhan), outside the submitted work. C.S. has served as 122 consultant, participated in advisory boards or received travel grants from AstraZeneca, Celgene,

123 Daiichi Sankyo, F. Hoffmann - La Roche Ltd, Genomic Health, Merck, Sharp and Dhome España 124 S.A., Novartis Odonate Therapeutics, Pfizer, Philips He. C. S. Verma \& S. Kannan are founders of Sinopsee Therapeutics and Aplomex; neither company has any conflict with the current work. VHIO has had funding (paid directly to the Institution) from AstraZeneca, Daiichi Sankyo, Eli Lilly and Company, Genentech, Immunomedics, Macrogenics, Merck, Sharp and Dhome España S.A., Novartis, Pfizer, Piqur Therapeutics, Puma, Roche, Synthon and Zenith Pharma. No potential conflicts of interest were disclosed by the other authors. 
151 Cyclin-dependent kinases 4 and 6 inhibitors (CDK4/6i), combined with endocrine therapy (ET),

152 have demonstrated higher antitumor activity than ET alone for the treatment of advanced estrogen

153 receptor-positive $\left(E R^{+}\right)$breast cancer $(B C)$. Some $E R^{+} B C$ are de novo resistant to $C D K 4 / 6 i$ and

154 others develop acquired resistance. Therapies for tumors after progression are needed. Here, we

155 demonstrate that $\mathrm{p} 16$ overexpression is associated with reduced antitumor activity of CDK4/6i in

156 patient-derived xenografts (PDX; $\mathrm{n}=37$ ) and $\mathrm{ER}^{+} \mathrm{BC}$ cell lines, and reduced response of

157 early/advanced ER+HER2- BC patients $(n=49)$ to $C D K 4 / 6 i$. We also identified heterozygous $R B 1$

158 loss as biomarker of acquired resistance and poor clinical outcome in ER+ ${ }^{+}$CDK4/6i-treated BC

159 PDX and patients. Combination of CDK4/6i ribociclib with PI3K inhibitor (PI3Ki) alpelisib showed

160 antitumor activity in $\mathrm{ER}^{+}$non-basal-like $\mathrm{BC} \mathrm{PDX}$, independently of PIK3CA or RB1 mutation

$161(n=25)$. Our results offer new insights into predicting primary and acquired resistance to CDK4/6i

162 and post-progression therapeutic strategies.

163

164

165

166

167

168

169

170

171

172

173

174

175

176

177

178

179 
184 The combination of cyclin D-dependent kinases 4 and 6 (CDK4/6) inhibitors (CDK4/6i) palbociclib, ribociclib and abemaciclib with endocrine therapy (ET) has been approved for the treatment of patients with advanced estrogen receptor-positive $\left(\mathrm{ER}^{+}\right)$and human epidermal growth factor receptor 2 (HER2)-negative breast cancer (BC) ${ }^{1-4}$. In the adjuvant setting, abemaciclib in combination with ET improves disease free survival compared with ET alone ${ }^{5}$. Abemaciclib or palbociclib in combination with trastuzumab and ET has also shown clinical activity in HER2positive $\left(\mathrm{HER} 2^{+}\right) / E R^{+} \mathrm{BC}{ }^{6,7}$. Despite the clinical success of these treatments in these subsets of $\mathrm{BC}$, the identification of biomarkers of response to CDK4/6i plus ET as well as designing novel therapeutic strategies for treating patients that escape from this therapy remains a major clinical need. Previous studies have highlighted that tumors sensitivity to CDK4/6 inhibition require a physiologically functional $\mathrm{G}_{1}-\mathrm{S}$ restriction point, as well as the absence of mechanisms that activate the cyclin E/CDK2 complex ${ }^{8-11}$. More specifically, high CDK6 results in a reduced response of phospho-pRb to CDK4/6 inhibitors ${ }^{12,13}$. Amplification and overexpression of FGFR1 has been associated with high expression of cyclin D1, resistance to antiestrogens alone and in combination with $\mathrm{CDK} 4 / 6 \mathrm{i}^{9}$. Loss of $\mathrm{pRb}$ itself implies the complete loss of cell cycle regulation at the $\mathrm{G}_{1-\mathrm{S}}$ restriction point for which $\mathrm{CDK} 4 / 6 \mathrm{i}$ are no longer effective ${ }^{14,15}$. Alternatively, high cyclin $\mathrm{E}$ results in resistance to CDK4/6i, as it bypasses the requirement of CDK4/6 for cell cycle progression ${ }^{16}$.

PI3K inhibitors (PI3Ki), in combination with fulvestrant, have been approved for the treatment of $\mathrm{ER}^{+}$metastatic $\mathrm{BC}$ with PIK3CA mutation ${ }^{17}$. The activity of combining PI3Ki and ET (NCT03056755) as well as triple combinations of CDK4/6i, PI3Ki and ET is being investigated in patients whose tumors progress after CDK4/6i treatment (NCT01872260; NCT02389842; NCT02088684; NCT03056755). In this study, we aimed to identify biomarkers of primary and acquired resistance to CDK4/6i in a panel of 37 patient-derived tumor models, using genetic, transcriptomic and proteomic approaches. Additionally, we explored if the combination of a PI3Ki plus a CDK4/6i has therapeutic potential in $\mathrm{ER}^{+}$and in $\mathrm{HER} 2^{+} \mathrm{BC}$ with primary or acquired resistance to $\mathrm{CDK} 4 / 6 \mathrm{i}$, in 
215 Ribociclib monotherapy has higher antitumor activity than other targeted agents in ER and HER2+ BC PDXs

217 Patient-derived xenografts (PDXs) are clinically relevant preclinical models for drug screening and biomarker identification ${ }^{18,19}$. We obtained 58 BC PDXs from implanting $473 \mathrm{ER}^{+} \mathrm{BC}$ tumor specimens in immune deficient mice (12\% of success rate; Figure $1 \mathrm{~A})$. Among the established PDXs, 12 from primary tumors and 9 from metastatic biopsies were initially available for the study (Table S1 and Table S2). Overall, the PDXs recapitulated the molecular subtypes of their corresponding original tumor, with the exception of PDX284, which lacked the expression of progesterone receptor (PR) and became TNBC (Table S1).

We then examined the antitumor activity of ribociclib in these 21 PDXs and responses to therapy were classified following the Response Evaluation Criteria In Solid Tumors (RECIST) criteria ${ }^{18,20}$. We observed one complete response (CR; 5\%), two partial responses (PR; 9.5\%), two stable diseases (SD; 9.5\%) and 18 progressive diseases (PD; 76\%; Figure 1B), for a total of $14 \%$ of preclinical response rate ( $p R R ; C R+P R)$ and a $24 \%$ of preclinical benefit rate ( $p C B ; C R+P R+S D)$. The three TNBC models were, as expected, refractory to CDK4/6 inhibition, whereas some of $\mathrm{ER}^{+}$and HER2 ${ }^{+}$PDXs responded and others did not. We subsequently tested the sensitivity of 17 available $\mathrm{ER}^{+}$or $\mathrm{HER}^{+} \mathrm{PDXs}$ to endocrine (fulvestrant or letrozole) or anti-HER2 (trastuzumab) therapies, respectively. We observed that PD was the best response in all but one case (PDX191 with SD on fulvestrant), including the 5 models sensitive to ribociclib (Figure 1C and Figure $\mathrm{S} 1 \mathrm{~A})$.

Resistance to ribociclib was generated from PDX244 (ER $\left.{ }^{+}, \mathrm{PDX} 244 \mathrm{LR}\right)$ after prolonged drug exposure (Figure 1D, ${ }^{8}$ ) and from PDX153 (HER2 ${ }^{+}$, PDX153LR), spontaneously after 7 serial passages in the absence of drug. Both these ribociclib-resistant models maintained the histopathological features of their respective sensitive counterparts (Table S1). In summary, we tested the sensitivity to ribociclib in 23 BC PDXs, including two models that acquired resistance to ribociclib from the sensitive counterparts.

\section{PDXs expressing high p16 are resistant to ribociclib}

To identify biomarkers of de novo or acquired resistance to ribociclib, we undertook genetic, transcriptomic and proteomic approaches. We firstly determined the intrinsic PAM50 subtype of 
the PDXs ${ }^{21}$. Most of the 23 PDXs showed concordant molecular and intrinsic subtypes (83\%) except four PDXs expressing ER, that were categorized as basal-like (PDX313, PDX098 and STG201) or HER2-enriched (PDX225) instead of Luminal B, suggesting that they are not dependent on ER signaling. Unsurprisingly, all basal-like models (ER ${ }^{+}$or TNBC) were resistant to ribociclib (Figure $2 \mathrm{~A} ;{ }^{22}$ ). We then performed genetic analyses of these PDXs employing a capture-based sequencing platform that detects genomic aberrations in $\sim 410$ cancer related genes (MSK-IMPACT ${ }^{\mathrm{TM}} ;{ }^{23}$ ) and analyzed whether the incidence of genetic alterations in thirteen cell cycle and PI3K-related genes correlated with ribociclib response $8-10,12,13,15,16$. We observed a trend towards ERBB2 amplification and $C D K N 2 A / B$ loss-of-function mutations being more frequent in ribociclib-sensitive PDXs, whereas CCND1 amplification and loss of function mutations in TP53 were identified amongst CDK4/6i-resistant models (Figure 2A, S1B and Table S3).

Comparing the mRNA expression levels of 54 cell cycle- and apoptosis-related genes in $12 \mathrm{ER}^{+}$ or HER2 ${ }^{+}$ribociclib-resistant versus 5 ribociclib-sensitive PDXs, we found that ribociclib-resistant models expressed higher CCNB2 $(p=0.002)$ as well as a trend towards higher CDK1 and CDK7 ( $p<0.1$; Figure S1C). Moreover, the pro-apoptotic genes BID and HRK were expressed at lower levels in ribociclib-resistant PDXs compared to the sensitive ones ( $p=0.03$ and 0.02 , respectively). A similar pattern of expression was observed in ribociclib-treated PDXs, with higher levels of CCNB2 $(p=0.03)$ and CCND1 $(p=0.14)$ in ribociclib-resistant PDXs (Figure S1D). These results suggest that CDK4/6i-resistant tumors harbor high CDK1/cyclin B2 activity and/or undergo early adaptation to non-canonical cell cycle bypass via CDK2/cyclin $\mathrm{D} 1^{8}$. At the protein level, both ribociclib-resistant and sensitive PDXs expressed comparable levels of ER, PR, CDK4, CDK6, cyclin D2, CDK2 and FGFR1 (Figure S2A). Of note, none of the PDXs included in this panel harbored high-level gene copy number (CN) of FGFR1 (Table S3; ${ }^{9,24}$ ). Conversely, higher $p 16$ levels $(p=0.01)$ and a trend towards low nuclear $p R b$ levels $(p=0.09)$ were detected in resistant PDXs compared to the sensitive ones (Figure 2B). In addition, even though the levels of cyclin E1 and cyclin D1 were similar in ribociclib-responders vs. non-responders $(p=0.2$ and 0.4 , respectively), PDXs expressing high levels of either protein were resistant to ribociclib. We further computed the accuracy of a complex biomarker composed of $p 16, p R b$, cyclin D1 and cyclin E1 expression. This composite marker showed higher sensitivity (87\%) and 
accuracy $(85 \%)$ for the detection of ribociclib-resistant models compared to single or binary biomarkers (Figure $2 \mathrm{C}$ ). Two out of $3 \mathrm{ER}^{+}$, basal-like PDX had high p16 levels with concomitant low pRb expression (PDX313, PDX098), which was expected given the described inverse relationship between $\mathrm{pRb}$ and $\mathrm{p} 16$ (Figure S2B) ${ }^{25-28}$. In addition, these models also expressed high cyclin E1, consistent with their basal-like intrinsic subtype ${ }^{21}$. In line with these observations, analysis of the TCGA dataset composed of $814 \mathrm{ER}^{+}$tumors, showed the co-occurrence between high p16 and low pRb, and low pRb and high cyclin E1 protein/mRNA expression ( $p=0.006$ and $p=0.02 / p<0.001$, respectively; Figure 2D). The co-occurrence between high $p 16$ and high cyclin E1 was only evidenced at the mRNA level $(p<0.001)$. Of note, up to $30 \%$ of $p 16$ high tumors in TCGA had normal levels of pRb and cyclin E1. In line with this, two out of 8 Luminal B PDX resistant to ribociclib (PDX039 and PDX287.2, 25\%) expressed high p16 without concomitant loss of $\mathrm{pRb}$ or overexpression of cyclin $\mathrm{E} 1$. This data suggests that high $\mathrm{p} 16$ protein expression is associated with CDK4/6i resistance both in models with non-functional pRb pathway, and in pRb normal tumors.

We further analyzed pharmacodynamic biomarkers by immunohistochemistry as in the PreOperative-Palbociclib (POP) trial ${ }^{29}$. We examined the percentage of Ki67- and phospho-pRb (Ser807/811)-positive cells in ribociclib-sensitive and -resistant PDXs without and with ribociclib treatment and observed a decrease of Ki67-positive cells as well as an unexpected increase of phospho-pRb-positive cells in ribociclib-sensitive PDXs (Figure S2C and S2D). In summary, our data show that the intrinsic subtype classification (PAM50) and an integrated biomarker composed of p16, pRb, cyclin E1 and cyclin D1 expression levels are associated with resistance to ribociclib in PDXs (Figure 2E).

\section{Biomarker validation in short-term patient-derived tumor cells (PDCs)}

To validate the potential predictive biomarkers for ribociclib antitumor activity, we measured the activity of ribociclib using patient-derived cells (PDCs) grown as short-term three-dimensional (3D) ex vivo cultures on a laminin-rich extracellular matrix (Figure $3 A$ ). Under the growing condition used, PDCs were able to proliferate for at least 14 days (Figure S2E). PDCs growth was monitored by measuring the spheroid area and the percentage of cells in the S-phase of the cell cycle using the 5-ethynyl-2'-deoxyuridine (EdU) incorporation assay. The antiproliferative 
activity of ribociclib was determined using sixteen representative available ex vivo cultures from the panel of 23 PDXs described above and showed that the responses to ribociclib of PDCs ex vivo agreed with that of their corresponding PDXs in vivo $(\mathrm{p}=0.0001$ and 0.021 ; Figure 3B, Figure S2F and S2G).

We then extended this analysis using ex vivo cultures from 14 additional ER ${ }^{+}$BC PDXs, three of which concomitantly expressing HER2 (Table S4). Based on p16, pRb, cyclin E1 and cyclin D1 expression levels, 9 of these 14 PDXs were predicted to be resistant and the remaining 5 sensitive to ribociclib (Table S4 and Figure 2E). Of note, 2 out of 9 models classified as ribociclib resistant due to high expression of p16 (PDX301 and PDX346) did not harbor loss of pRb. As expected, PDCs predicted as ribociclib sensitive exhibited a greater reduction in the relative spheroid area upon treatment than PDCs predicted as ribociclib-resistant $(p=0.005$; Figure $3 C)$. Moreover, with the exception of PDX350 and PDX399, two models with unexpectedly high ex vivo sensitivity, the responses to ribociclib of all PDCs were in agreement with the predicted responses (Table S4 and Figure 3D). ROC curve analysis indicated that the change of the spheroid area upon ribociclib treatment could discriminate between sensitive and resistant PDCs with $100 \%$ sensitivity and $87.5 \%$ specificity $(p<0.0001$; cut-off $=-25 \% ; n=37$ models; Figure $3 E$ ). In summary, also using PDCs in ex vivo cultures we could identify ribociclib resistant $\mathrm{ER}^{+} \mathrm{BC}$ tumors using a complex biomarker composed of $\mathrm{p} 16, \mathrm{pRb}$, cyclin $\mathrm{E} 1$ and cyclin $\mathrm{D} 1$.

\section{p16 and cyclin D1 overexpression attenuate the response to ribociclib in $\mathrm{ER}^{+} \mathrm{BC}$ cell lines}

Previous studies have already demonstrated the impact of RB1 loss and CCNE1 amplification in the response to CDK4/6i $8,13,15,16$. Therefore, we aimed at evaluating whether $p 16$ and cyclin $D 1$ overexpression can be added to the list of candidates associated with resistance to ribociclib in $\mathrm{ER}^{+} \mathrm{BC}$. We generated T47D cells $\left(E R^{+}\right)$overexpressing $\mathrm{p} 16$ under doxycycline regulation (T47Dp16). We measured their response to ribociclib, fulvestrant and the combination and found that T47D-p16 cells had 4.5- to 20.4-fold higher IC 50 (half-maximal inhibitory concentration) than MOCK control cells (Figure 4A and Figure S3A). Biochemical analysis revealed that T47D-p16 cells had higher levels of phospho-pRb (S780, $p=0.0004$; S807/811, $p=0.003$ ), cyclin E2 $(p=0.003)$ and phospho-CDK2 T160 $(p=0.0009)$ compared to control cells (Figure 4B and Figure S3B). In a competition experiment, whereby p16-overexpressing cells were seeded 1:20 with 
control cells, $p 16$ expression levels were upregulated after 14 days of treatment $(p=0.0006$; Figure 4C and Figure S3C). This result suggests that pre-existing, low-abundant p16-overexpressing cells were positively selected upon treatment with CDK4/6i and represent a reservoir of drug resistant cells.

To further support these findings, we posited that P18IN003 ${ }^{30}$, an inhibitor of the p18-CDK4 interaction, would also impair the binding of $\mathrm{p} 16$ to CDK4 and sensitize p16-high PDCs to CDK4/6i. Using in silico modeling, we observed that the ankyrin repeats 1, 2, 3 in p16 constitute a binding pocket that is relatively large and shallow. One part of this pocket consists largely of hydrophobic residues (Val51, Met52, Met53 and Met54) and the other part contains charged residues (Asp74, Asp84, Glu88, Arg46 and Arg87). P18IN003 fits into this pocket, with one methoxyphenyl moiety and the dihydroimidazole moiety of P18IN003 involved in H-bond interactions with Asp74 and Glu88, respectively, of p16. The other methoxyphenyl moiety of P18IN003 is solvent exposed. Comparing the predicted p16-P18IN003 interaction with the in silico model of the p16-CDK4 interaction revealed that P18IN003 would disrupt the binding of CDK4 to $\mathrm{p} 16$ by binding to this pocket (Figure 4D). Indeed, in ex vivo cultures P18IN003 combined with ribociclib markedly reduced proliferation in PDC191 (p16-high, pRb-expressing model; $p=0.0009$ ) but not PDC313 ( $p 16$ high, $p R b$ low model; $p=0.2$ ) compared to ribociclib monotherapy (Figure 4E), presumably due to increased binding of ribociclib to CDK4 ${ }^{31}$.

The response to ribociclib, fulvestrant and their combination was also evaluated in T47D and MCF7 cells overexpressing cyclin D1 (T47D-cyclin D1 and MCF7-cyclin D1). Cyclin D1 overexpression moderately increased the $\mathrm{IC}_{50}$ values 3.1- to 5.0-fold in T47D and 2.3- to 3.3-fold in MCF7 (Figure 4F and Figure S3D). In line with this, T47D and MCF7 cells overexpressing cyclin D1 showed an attenuated response to downmodulation of phospho-pRb, cyclin E2 and phosphoCDK2 T160 upon treatment with ribociclib (Figure 4G and Figure S3E). In a competition experiment, cyclin D1 expression was upregulated after 14 days of treatment in both T47D $(p=0.0004)$ and MCF7 cell lines $(p=0.01$; Figure $4 \mathrm{H}$ and Figure S3F). This result suggests that pre-existing, low-abundant cyclin D1-overexpressing cells were positively selected upon treatment with CDK4/6i and represent a reservoir of drug resistant cells. In summary, we conclude that overexpression of either $p 16$ or cyclin $D 1$ attenuates the response of $B C$ cells to CDK4/6i through activation of $\mathrm{G} 1$ checkpoint kinase activity. 
Given our preclinical results, we interrogated a potential association of the aforementioned biomarkers with response to CDK4/6i in $\mathrm{ER}^{+} \mathrm{BC}$ patients. In early-breast cancer, palbociclib and abemaciclib treatment significantly reduced proliferation and the CDK4/6 downstream response, respectively measured by Ki67 and phospho-pRb (S807/811) ${ }^{29} 32$. Here, we reanalyzed data from the abemaciclib preoperative (ABC-POP) clinical trial ${ }^{32}$. In total, 72 patient samples were analyzed, 33 Luminal A and 39 Luminal B. As expected, the majority of the Luminal A tumors were sensitive to CDK4/6 inhibition in terms of drop of Ki67 at day 15 of treatment, a "gold standard" biomarker of endocrine sensitivity in this patient population ${ }^{33}$. On the contrary, we found that similar proportions of Luminal B tumors could be classified as responders (56\%) and nonresponders (44\%; Figure 5A). In line with results obtained in PDXs (Figure 2B), the $\mathrm{H}$-score levels of $\mathrm{p} 16$, but not $\mathrm{pRb}$ and cyclin $\mathrm{D} 1$, were significantly higher in resistant tumors treated with abemaciclib compared to sensitive Luminal B tumors ( $p=0.008$; Figure 5B and Figures S3G-H). High p16 H-score levels were significantly associated with the tumor's response to CDK4/6 inhibition by abemaciclib in the ABC-POP trial $(p=0.008$, Figure $5 C)$. Next, we tested if high p16 was also associated with lack of response to abemaciclib as single agent in the metastatic setting $(n=10)$. Higher $p 16$ levels $(p=0.04)$ and a trend towards high cyclin E1 levels $(p=0.1)$ were detected in resistant tumors compared to the sensitive ones, whereas levels of $p R b$ and cyclin D1 were similar between both groups (Figure 5D). In this cohort, p16 expression $(p=0.02)$, but not the complex biomarker, was associated with the response to abemaciclib (Figure 5E). In summary, our data shows that high protein levels of p16 are associated with resistance to $\mathrm{CDK} 4 / 6 \mathrm{i}$ in both primary and metastatic $\mathrm{ER}^{+} \mathrm{BC}$ tumors.

Acquisition of subclonal RB1 mutations as mechanism of acquired resistance to ribociclib in tumors with RB1 heterozygous loss

We next posited that tumors with an underlying $R B 1$ heterozygous loss tend to acquire $R B 1$ point mutations that result in CDK4/6i resistance. We therefore generated eight derivatives from PDX244 that became refractory to ribociclib treatment overtime ${ }^{8}$. Sequencing data from the sensitive tumor $(2 \mathrm{R})$ revealed a CDKN2A homozygous loss and concomitant $R B 1$ heterozygous loss (Figure 6A). Protein analysis confirmed the lack of $\mathrm{p} 16$ and normal $\mathrm{pRb}$ levels in this model 
(Figure 6A). Three out of 8 ribociclib resistant tumors (16L, 16R and 18R) acquired deleterious mutations in RB1 (p.M695Nfs*26, p.K810* and p.X180_splice, respectively) and tumors $19 \mathrm{~L}$ and $19 \mathrm{R}$ underwent a further reduction in the $R B 1$ copy number (from -0.9 to -1.5 and -2.0 , respectively), suggesting the acquisition of homozygous RB1 loss. Protein analysis by IHC confirmed the total or sub-clonal loss of pRb expression in these tumors (16L and $19 \mathrm{~L}$ vs. $16 \mathrm{R}$ and $18 \mathrm{R})$. Intriguingly, a sixth tumor (15R) also showed partial loss of $\mathrm{pRb}$ expression without a detectable underlying genetic alteration in $R B 1$, suggesting alternative mechanisms that regulate the RB1 gene expression. Regarding $15 \mathrm{~L}$ and $17 \mathrm{~L}$, we observed an increment in CDKN2A CN log ratio (from -4.2 to -0.4 ) along with the restoration of $p 16$ expression (Figure $6 \mathrm{~A}$ ), suggesting that selection of tumor cells retaining normal $C D K N 2 A C N$ was favored upon treatment. Cyclin E1 and cyclin D1 did not show any alteration at the gene or protein levels in any of the acquiredresistant tumors (Figure 6A). In addition to the aforementioned model, we developed paired PDXs from a patient who received palbociclib plus letrozole before treatment initiation (PDX476.1) and after 12 cycles of treatment at the time of disease progression (PDX476.2). Similar to their respective original tumors, PDC476.1 was sensitive to palbociclib whereas PDC476.2 was resistant (Figure S4A). Genetic analysis showed that PDX476.1 harbored a heterozygous $R B 1$ loss $(\mathrm{CN}=0.9)$ but still expressed pRb. In contrast, PDX476.2 lost pRb protein expression, suggesting that it may be the mechanism responsible of tumor progression (Figure 6B). Next, we interrogated the prognostic implications of $R B 1$ heterozygous loss in $\mathrm{ER}^{+} \mathrm{BC}$ patients. In two out of the three cohorts analyzed, patients with tumors harboring RB1 heterozygous loss showed significantly poorer clinical outcome compared to patients with unaltered $R B 1$ tumors in terms of disease-free survival (DFS), overall survival (OS) or days of treatment (DOT; Figure 6C). In order to analyze the role of $R B 1$ heterozygous loss as biomarker of resistance to CDK4/6i in patients, we obtained genomic and clinical data of metastatic BC from patients included in the Hartwig Medical Foundation (HMF) cohort ${ }^{34}$. Out of 582 patients diagnosed with metastatic BC, 71 received CDK4/6i. To test whether concomitant heterozygous deletion and mutation of $R B 1$ gene appears preferentially amongst patients who had received CDK4/6 inhibitors, we applied a multivariable logistic regression. We found a significant association between the double hit (mutation and heterozygous deletion) and the prior exposure of the patient to CDK4/6i as part of 
their treatment $(p=0.003$, Figure $6 D)$. No significant association was found between any alteration

426 (only mutation, only deletion or both) affecting RB1 and the previous exposure to CDK4/6

427 inhibitors (Figure S4B), implying that only patients with a double hit in RB1 have an increased likelihood of having received CDK4/6i. Altogether, these data suggest that tumors harboring heterozygous $R B 1$ loss are susceptible of acquiring a second hit in the RB1 gene and becoming resistant to CDK4/6i.

\section{The PI3K inhibitor alpelisib sensitizes non-basal like BC PDX to ribociclib} Preclinical studies ${ }^{35}$ and a phase III clinical study (SOLAR-1, NCT02437318, ${ }^{17}$ ) have shown that PI3K- $\alpha$ inhibitors, such as alpelisib, are able to sensitize PIK3CA-mutant tumors to endocrine therapy. Currently, this approach is further being tested in the context of tumors that progress after being treated with CDK4/6i-containing regimens (e.g. BYlive, NCT03056755, ${ }^{36}$ ). Here, we interrogated whether alpelisib can sensitize ribociclib-resistant PDXs to CDK4/6i. In our PDX panel, alpelisib monotherapy resulted in a pCB of $43 \%$ compared to $25 \%$ of ribociclib alone (Figure 7A). All HER2 ${ }^{+}$PDXs tested were sensitive to alpelisib, including the ribociclibresistant PDX153LR, PDX222 and PDX118. All the PIK3CA mutated tumors were sensitive to alpelisib except PDX287.3, which was derived from a patient's tumor that progressed while being treated with the PI3Ki GDC-0032 plus letrozole (Figure S4C). As expected, all 3 TNBC PDXs were resistant to alpelisib since none of them harbored a PIK3CA mutation.

444 Combined treatment of ribociclib and alpelisib resulted in a pCB of 78\% (Figure 7A). We noticed that all basal-like PDXs by PAM50 exhibited PD or SD as best response. Similarly, combination of palbociclib plus GDC-0032 also showed improved antitumor activity compared to either one alone in PDX287.2 and PDX287.3 that derived from a patient's tumors collected on-treatment or after progression with GDC-0032 plus letrozole, respectively (Figure S4D). Analysis of pharmacodynamic biomarkers showed that both Ki67 and phospho-pRb S807/811 were downregulated in PDXs that responded to the drug combination $(p<0.001$ and $p<0.01$, respectively), but not in the resistant ones (Figure 7B). Because of concerns regarding the safety of the combination of alpelisib plus ribociclib, we conducted de-escalation experiments showing that dose reduction of either ribociclib or alpelisib resulted in similar antitumor activity as the full 
dose tested in both PDX039 and PDX191 models. Dose reduction of both drugs, however, resulted in an attenuated efficacy (Figure S4E).

456 Importantly, we demonstrated that ribociclib plus alpelisib (or the triple combination with

457 fulvestrant) was effective in PIK3CA-wt PDXs with primary resistance to ribociclib plus fulvestrant 458 (PDX131 and PDX244LR\#18R, Figure 7C-D) or in a PDX from a patient who showed an early progression when treated with palbociclib plus letrozole (PDX450, PIK3CA and ESR1 mutant; Figure 7E). Similar results were performed ex vivo with the PDX (PIK3CA-wt) from a patient who received palbociclib plus letrozole: treatment of PDC476.2 with palbociclib plus alpelisib resulted in higher reduction of the spheroid area than palbociclib plus fulvestrant (Figure S4A). The triple combination of ribociclib plus alpelisib and fulvestrant was the most efficient in controlling proliferation and, biochemically, increasing the levels of PARP1 cleavage (Figure 7F). Altogether, these results suggest that the combination of a PI3Ki with a CDK4/6i, with or without $E T$, is a valid therapeutic option for the treatment of $\mathrm{ER}^{+} \mathrm{BC}$ tumors after progression on $\mathrm{CDK} 4 / 6 \mathrm{i}$ plus $\mathrm{ET}$, independently of PIK3CA mutation status or $\mathrm{pRb}$ expression.

468 


\section{DISCUSSION}

Fifteen to $30 \%$ of $\mathrm{ER}^{+}$metastatic $\mathrm{BC}$ progress rapidly when treated with $\mathrm{CDK} 4 / 6 \mathrm{i}$ plus $\mathrm{ET}$. In general, the subsequent line of therapy has a short duration, lasting less than 6 months ${ }^{37-39}$. Therefore, it is important to identify the group of patients that are not likely to benefit from CDK4/6i plus ET to avoid unnecessary toxicity, to reduce unnecessary costs and to provide more effective alternative treatments. Targeting mTORC1 with everolimus or PI3K with alpelisib in combination with aromatase inhibitors (Al) or fulvestrant, respectively, are currently available therapies but have some limitations. Everolimus approval for metastatic $\mathrm{ER}^{+} \mathrm{BC}$ is in combination with an $\mathrm{Al}$, but $\mathrm{Al}$ is not active in patients with ESR1-mutant tumors. On the other hand, alpelisib is only recommended for patients with $\mathrm{BC}$ harboring PIK3CA mutations ${ }^{17,40}$. In addition, there is very limited evidence of activity of these regimens after relapsing to a CDK4/6i-based treatment ${ }^{17,36}$. In this work, we explored biomarkers for resistance to CDK4/6i and the efficacy of a therapeutic strategy based on CDK4/6i plus PI3Ki. We identified that overexpression of p16 or cyclin D1 is associated with an impaired response to CDK4/6i and that a CDK4/6i in combination with a PI3Ki is effective in CDK4/6i-resistant $\mathrm{ER}^{+}$, non-basal like PDXs, independent of the PI3KCA mutation status. Moreover, triplet combinations of a CDK4/6i with a PI3Ki and ET are active in PDXs harboring biallelic $R B 1$ mutation (or $\mathrm{pRb}$ protein loss).

501 Although CDK4/6i are usually administrated in combination with ET, abemaciclib can be used as a single agent for the treatment of patients with $E R^{+} / \mathrm{HER} 2$-negative metastatic $\mathrm{BC}$ that progressed on ET and chemotherapy ${ }^{4}$. Also, the data from the TREnd trial suggested that CDK4/6i combined with ET and CDK4/6i monotherapy had similar clinical benefit rates and overall response rates in post-menopausal women with advanced ET-pretreated ER ${ }^{+} / \mathrm{HER} 2$-negative $\mathrm{BC}$ ${ }^{41}$. In this sense, our data demonstrates that targeting the CDK4/6 axis is a valid strategy when other targeted therapies against ER or HER2 have failed ${ }^{4,42,43}$. We observed that $C D K N 2 A / B$ were more frequently disrupted in ribociclib-sensitive models and the best PDX responder lacked expression of $\mathrm{p} 16$. Similarly, in the phase I trial of abemaciclib the best BC responder harbored a concomitant deletion of $C D K N 2 A$ and $C D K N 2 B$ in her tumor ${ }^{43}$. This observation is in line with the limited capacity of current CDK4/6i to inhibit its target when it is bound to $p 16^{31,44}$ and consistent with the increased sensitivity to palbociclib observed in 544 cancer cell lines in association with CDKN2A inactivation by DNA methylation ${ }^{45}$. 
FGFR1 gain and TP53 mutation detected in circulating tumor DNA have recently emerged as markers of early progression on CDK4/6i treatment, albeit they are also associated with poor prognosis irrespective of palbociclib treatment ${ }^{9,24}$. Although CCND1 and CDKN2A individually have not been associated lack of with response to CDK4/6i plus ET, results from the BioltaLee (NCT03439046) and PALOMA-1 (NCT00721409) trials suggest that genes of the CDK4/6 pathway are also biomarkers of de novo resistance ${ }^{2,46}$.

High p16 protein has been previously described as a surrogate biomarker of pRb loss in several tumor types ${ }^{25,26}$. Here, we found similar results analyzing $814 \mathrm{ER}^{+}$invasive breast carcinomas from the TCGA dataset. However, we also observed that $30 \%$ of the p16-high tumors from TCGA are not associated with low $\mathrm{pRb}$ expression, suggesting that $\mathrm{p} 16$ might independently predict CDK4/6i-resistance. This phenotype might have been relevant in CDK4/6i-resistant tumors from the ABC-POP trial ${ }^{32}$ in which $\mathrm{p} 16$, but not $\mathrm{pRb}$, was associated with resistance to abemaciclib. Our data show that high $\mathrm{p} 16$, in addition to preventing the binding of CDK4/6i to its target ${ }^{31}$, can facilitate the $\mathrm{G} 1$ checkpoint bypass via cyclin E1/CDK2 or via non-canonical cyclin D1/CDK2, which was previously described as an adaptive mechanism to $\mathrm{CDK} 4 / 6 \mathrm{i}$ in $\mathrm{ER}^{+} \mathrm{BC}$ cell lines ${ }^{8}$. In this sense, our data also provides evidence that impairing p16-CDK4/6 binding with a INK4 inhibitor restores sensitivity to CDK4/6i. Similar results were obtained in cell lines overexpressing cyclin D1 which exhibited an attenuated response to ribociclib treatment and residual cyclin E2CDK2 activity upon treatment. Acquisition or pre-existing RB1 mutations has been associated with resistance to HT plus CDK4/6i ${ }^{13,15,24}$. Wander et al. described the biallelic RB1 disruption in two separated samples from a patient progressing to $\mathrm{HT}$ plus palbociclib, who harbored an $R B 1$ heterozygous loss in her pre-treatment tumor sample ${ }^{47}$. These evidences are in line with our results showing that most of the tumors from the ribociclib-sensitive PDX244 became resistant to CDK4/6 blockade due to acquisition of a second hit in RB1. In patients, we observe that $R B 1$ heterozygous loss is associated with worse prognosis and that a double hit in $R B 1$ was more likely to occur in patients who received a CDK4/6i that in those with ET alone. At least in part, this might be explained by the haploinsufficiency of $p R b$ in its contribution to DNA repair in the S-phase checkpoint and initiation of DNA replication; cancer cells with only one copy of $R B 1$ exhibit a genomic instability phenotype ${ }^{48}$. Therefore, tumors harboring RB1 heterozygous loss might be prone to acquire a 
second hit in $R B 1$ and resistance to CDK4/6 blockade. As we have shown, tumors with $R B 1$ loss are sensitive to the triplet combination of CDK4/6 plus $\mathrm{PI} 3 \mathrm{Ki}$ and $\mathrm{HT}$ or might be sensitive to other targeted treatments such as inhibitors of the Aurora kinases AURKA and AURKB ${ }^{49}$. In closing, therapies for metastatic BC that progress on or after CDK4/6i treatment currently being tested in the clinic include: 1) ET or more potent SERDs in endocrine sensitive patients, albeit this patient population is difficult to identify (NCT02338349, NCT03616587); 2) continuation of CDK4/6i with a different ET backbone (NCT03616587; NCT03809988); 3) continuation of ET with a different CDK4/6i; for example with abemaciclib, because it has additional targets including CDK1/2 complexes ${ }^{50} ; 4$ ) different ET combined with a PI3Ki in PI3KCA mutant ER ${ }^{+} \mathrm{BC}^{17,36}$; or 5) different ET combined with an AKT inhibitor in PIK3CA/AKT1/PTEN altered tumors (NCT04305496). In this context, our preclinical data previously reported suggest that PTEN alterations alone do not result in benefit to AKT inhibitors ${ }^{51}$. Here, we report that the combination of ribociclib with the PI3K inhibitor alpelisib (or palbociclib with GDC-0032) has remarkable antitumor activity in non-basal like PDXs independent of PIK3CA mutation and this observation is novel ${ }^{11,52}$. Of note, PAM50-based intrinsic subtyping has become a potential indicator of benefit to CDK4/6i ${ }^{53-55}$. In addition, CDK4/6i plus PI3Ki and ET demonstrated antitumor efficacy in PDXs harboring $R B 1$ mutation and it may be an appropriate first line treatment strategy for patients harboring heterozygous $R B 1$ loss $^{47}$. Altogether, this study identifies high p16 protein levels and heterozygous $R B 1$ loss as novel biomarkers for resistance to CDK4/6i treatment and suggests that CDK4/6i plus PI3Ki may be effective in non-basal-like tumors that progress to CDK4/6i and ET, independently of the PIK3CA or RB1 status. 
576 This study was designed to unravel predictive biomarkers of response to ribociclib that can be 577 effectively used for patient stratification. We assessed ribociclib activity in a cohort of 37 patient578 derived xenograft models from primary/metastatic breast cancer patients. All animal procedures were approved by the Ethics Committee of Animal Research of the Vall d'Hebron Institute of

580 Oncology and by the Catalan Government and were conformed to the principles of the WMA Declaration of Helsinki, the Department of Health and Human Services Belmont Report and following the European Union's animal care directive (2010/63/EU). For ethical issues, in vivo experiments were ended when the total tumor volume of a mouse surpassed $1500 \mathrm{~mm}^{3}$ or a decline in mouse welfare was observed. Tumors were harvested and formaldehyde and flashfrozen for posterior proteomic and genomic analyses.

We obtained fresh and formalin-fixed paraffin embedded (FFPE) tumor samples from the Vall d'Hebron University Hospital and following the institutional guidelines. Informed written patient consent, approved by the Ethics Committee for Clinical Research and Animal Research of Vall d'Hebron Hospital, was obtained for the use of these patient samples. Use of PDXs from other laboratories was approved by the National Research Ethics Service, Cambridgeshire 2 REC (RED reference number: 08/H0308/178 ((44) and http://caldaslab.crik.cam.ac.uk/bcape/) or by the Central Office for Research Ethics Committee study number 05/Q1402/25.

\section{Generation of PDXs}

Tumor pieces of 30 to $60 \mathrm{~mm}^{3}$ obtained from patient primary tumors or metastatic lesions at time of biopsy were immediately implanted into the mammary fat pad (surgery samples) or the lower flank (metastatic samples) of 6-week-old female NOD.Cg-Prkdc ${ }^{\text {scid }} / / 2 \mathrm{rg}^{\mathrm{tm} 1 W_{\mathrm{j}} /} / \mathrm{SzJ}$ mice (Charles Rives). Animals were housed in air-filtered flow cabinets with a 12-hours light cycle and food and water ad libitum. Mice were continuously supplemented with $1 \mu \mathrm{mol} / \mathrm{L} 17 \beta$-estradiol (SigmaAldrich) in their drinking water, an amount shown to be sufficient to reach serum levels and uterine growth in ovariectomized female mice similar to the ones obtained with other mechanism of $17 \beta-$ estradiol supplementation ${ }^{56}$. Upon growth of the engrafted tumors, a tumor piece was implanted into the lower flanks of new recipient mice for the model perpetuation. In each passage, flash- 
604

605

606

607

608

609

610

611

612

613

614

615

616

617

618

619

620

621

622

623

624

625

626

627

628

629

630

631

632

633

frozen and formalin-fixed paraffin embedded (FFPE) samples were taken for genotyping and histological studies. STG201 was generated in CRUK/UCAM as previously reported ${ }^{19}$ and PDXs BB3RC31, BB6RC39, BB6RC87 and BB6RC160 were generated in Manchester Breast Center as previously reported ${ }^{57}$. Both laboratories are members of the EuroPDX consortium (http://www.europdx.eu).

\section{In vivo experiments}

To evaluate the sensitivity to the different targeted therapies each PDX was implanted subcutaneously in six-week-old female athymic nude HsdCpb:NMRI-Foxn1nu mice (Janvier) or NOD.Cg-Prkdcscid/l2rg ${ }^{\text {tmiwil}} / S z J$ mice (Charles Rives) and supplemented with $1 \mu \mathrm{mol} / \mathrm{L} 17 \beta-$ estradiol (Sigma-Aldrich) in their drinking water as previously described. Upon xenograft growth, tumor-bearing mice were randomized into treatments group with tumors ranging $100-300 \mathrm{~mm}^{3}$ (for drug efficacy experiments) or $\sim 500 \mathrm{~mm}^{3}$ (for short-term pharmacodynamic experiments). Ribociclib was administrated by oral gavage once daily, six days/week, at $75 \mathrm{mg} / \mathrm{kg}$ (total daily dose) dissolved in distilled water $0.5 \%$ hydroxymethyl cellulose. Alpelisib was dosed with the same schedule at $35 \mathrm{mg} / \mathrm{kg}$ dissolved in distilled water $0.5 \%$ methylcellulose. The combination was administrated with one-hour delay between ribociclib (first) and alpelisib (second). Fulvestrant was administered subcutaneously twice weekly $10 \mathrm{mg} / \mathrm{mice}$ dissolved in peanut-oil, letrozole by oral gavage three times per week ( 1 day on and 1 day off) $20 \mathrm{mg} / \mathrm{kg}$ dissolved $0.5 \%$ methylcellulose and trastuzumab by intraperitoneal injection twice weekly $10 \mathrm{mg} / \mathrm{kg}$ in PBS.

Tumor growth was measured bi-weekly blinded to the treatment effect with a caliper the first day of treatment and to day 35 (for the efficacy assays of ribociclib, alpelisib and their combination), day 15 (for the efficacy assays of fulvestrant and trastuzumab) or day 12 (pharmacodynamic assays). Mice weights was recorded twice weekly. The tumor volume was calculated using the ellipsoid formula: $V=\left(\right.$ length $x$ width $\left.^{2}\right) \times(\pi / 6)$. Mice were euthanized when tumors reached 1500 $\mathrm{mm}^{3}$ or in case of severe weight loss, in accordance with institutional guidelines. All the efficacy experiments contained an untreated control arm with a percentage of change in tumor growth superior to $20 \%$ from the initial volume. The antitumor activity was determined by comparing tumor volume at last day of treatment to its baseline (day1): \% tumor volume change $=\left(V_{35 \text { days }}-\right.$ $V_{\text {baseline }} / V_{\text {baseline }} \times 100$. To classify the antitumor response of subcutaneous implants, the 
Response Evaluation Criteria in Solid Tumors (RECIST) based on the \% tumor volume change was modified and labeled as mRECIST ${ }^{18,20}$. Complete response (CR) was set as best response $\leq-95 \%$; partial response (PR) as $-95 \%<$ best response $\leq-30 \%$; stable disease (SD) as $-30 \%<$ best response $\leq+20 \%$; and progressive disease $(P D)$ as best response $>+20 \%$. The models that displayed a preclinical benefit from ribociclib (SD, PR, and $C R$ ) were categorized as ribociclibsensitive. All PD models were categorized as ribociclib-resistant. At the end of the experiment, animals were euthanized using $\mathrm{CO}_{2}$ inhalation. Tumor volumes are plotted as means and $\pm \mathrm{SEM}$.

\section{Generation of ribociclib resistant models}

Ribociclib-sensitive models were chronically treated with ribociclib until progression. Tumor growth was measured once per week and mice weights were recorded twice per week. If mouse welfare was compromised before tumor progression, tumors were harvested and implanted into another recipient mouse. Dosing schedule was reinitiated 10 days post-surgery and lasted until progression.

\section{Molecular subtype of PDXs}

Immunohistochemical staining was performed on formalin-fixed paraffin embedded (FFPE) PDXs tissue sections $(3 \mu \mathrm{m})$. Staining of estrogen receptor $(E R)$, progesterone receptor $(P R)$ and human epidermal growth factor receptor 2 (HER2) were undertaken following the protocol provided by Ventana Medical Systems, Inc. In short, the slides were heated in the oven at $75^{\circ} \mathrm{C}$ for 28 minutes and deparaffinized with EZ prep solution (Ventana Medical Systems). Then, antigen retrieval was performed at slightly basic $\mathrm{pH}$ at $95^{\circ} \mathrm{C}$ for $56 \mathrm{~min}$. Primary antibodies were incubated for $40 \mathrm{~min}$ for ER and HER2 using the Cell Conditioning 1 buffer (CC1; Ventana Medical Systems), and with CC2 buffer for the PR antibody. Finally, the slides were counterstained with Hematoxylin II and Bluing Reagent (Ventana Medical Systems) and mounted with xylol based mounting medium. An investigator blinded to identify the samples quantified the percentage of positively stained cells.

\section{PAM50 intrinsic molecular subtype assignment}

Flash-frozen pieces of tumor xenograft were used for RNA sequencing and PAM50-molecular subtype classification as previously described ${ }^{58,59}$. All the tumor samples used in this study were 
pieces of patient-derived xenografts. After surgical resection, the tumors were dissected, and a piece was quickly frozen in liquid nitrogen and stored at $-80^{\circ} \mathrm{C}$. A frozen tumor specimen was then homogenized in RNAse-free containing lysis buffer and mRNA was prepared by using a PerfectPure RNA Tissue Kit-50 from 5 Prime and protocol.

$250 \mathrm{ng}$ of total RNA were used to measure the expression of 50 genes of the PAM50 intrinsic subtype predictor assay and 5 housekeeping genes (ACTB, MRPL19, PSMC4, RPLPO, and SF3A1) using the nCounter platform (NanoString Technologies). Data was log base2-transformed and normalized to the housekeeping genes using the nSolver 4.0 software and custom scripts in R 3.4.3 software. All PDX tumors were assigned to an intrinsic molecular subtype of breast cancer (Luminal A, Luminal B, HER2-enriched, Basal-like or Normal-like) using the PAM50 subtype predictor ${ }^{60}$.

\section{Targeted exome sequencing (MSK-IMPACT ${ }^{\mathrm{TM}}$ )}

Flash-frozen pieces of tumor xenografts were used for DNA sequencing by the MSK-IMPACT ${ }^{\mathrm{TM}}$ (Integrated Mutation Profiling of Actionable Cancer Targets), a hybridization capture-based nextgeneration sequencing assay for targeted deep sequencing designed to capture all protein-coding exons and selected introns of 410 commonly implicated oncogenes, tumor suppressor genes, and members of pathways deemed actionable by targeted therapies ${ }^{23}$. Barcoded sequence libraries were prepared using 100-250 ng genomic DNA (Kapa Biosystems) and combined into equimolar pools of 13-21 samples. The captured pools were subsequently sequenced on an Illumina HiSeq 2000 as paired-end 100-base pair reads, producing a median of 588-fold coverage per tumor. Sequence data were demultiplexed using CASAVA, and reads were aligned to the reference human genome (hg19) using BWA and post-processed using the Genome Analysis Toolkit (GATK) according to GATK best practices. MuTect and GATK were used to call singlenucleotide variants and small indels, respectively. Candidate mutations were manually reviewed using the Integrative Genomics Viewer (IGV) to eliminate likely false positive calls. Because matched normal DNA was not available, tumors were compared to a pool of unmatched normal samples to eliminate common polymorphisms and systematic sequencing artifacts. 2 
Patient-derived tumor cells (PDC) were isolated from PDX through combination of mechanic disruption and enzymatic disaggregation following the protocol described by Bruna et al ${ }^{19}$. Briefly, PDX tumors not bigger that $500 \mathrm{~mm}^{3}$ were freshly collected in DMEM/F12/HEPES (GIBCO) after surgery resection, minced using sterile scalpels and dissociated for a maximum of 90 minutes in DMEM/F12/HEPES (GIBCO), $1 \mathrm{mg} / \mathrm{ml}$ collagenase (Roche), $100 \mathrm{u} / \mathrm{ml}$, hyaluronidase (SigmaAldrich), $5 \%$ BSA (Sigma-Aldrich), $5 \mu \mathrm{g} / \mathrm{ml}$ Insulin and $50 \mu \mathrm{g} / \mathrm{ml}$ gentamycin (GIBCO). This was followed by further dissociation using trypsin (GIBCO), dispase $5 \mathrm{mg} / \mathrm{ml}$ (StemCell technologies) and DNase $1 \mathrm{mg} / \mathrm{ml}$ (Sigma-Aldrich). Red blood cell lysis was done by washing the cell pellet with 1X Red Blood Cell (RBC) Lysis Buffer containing ammonium chloride (Invitrogene). Then, cells were resuspended in MEGM ${ }^{\mathrm{TM}}$ Mammary Epithelial Cell Growth Medium Bulletkit ${ }^{\mathrm{TM}}$ (LONZA) supplemented with $2 \%$ of fetal bovine serum and $10 \mu \mathrm{M}$ of ROCK inhibitor (Sigma-Aldrich). To test drug antiproliferative responses and for Western-Blot analysis, cells were seeded on collagen-enriched matrix Corning ${ }^{\circledR}$ Matrigel $₫$ growth factor reduced (GFR) basement membrane matrix (Corning, INC) at $2 \times 10^{5}$ cells $/ \mathrm{ml}$ in 8 well-chamber slides (NUNC) or $1 \times 10^{6}$ cells $/ \mathrm{ml}$ in 6 well-plates (BD Biosciences), respectively. The following day, PDCs were treated with vehicle (DMSO), $1 \mu \mathrm{M}$ of ribociclib, 500nM palbociclib, $2.5 \mu \mathrm{M}$ alpelisib, 100nM fulvestrant, $20 \mu \mathrm{M}$ $\mathrm{P} 18 \mathrm{IN} 003$ or the combinations and cultured at $37^{\circ} \mathrm{C}$ in $5 \%$ of $\mathrm{CO}_{2}$. Medium and treatments were refreshed every 2-3 days.

\section{Analysis of PDCs area}

Cell suspensions generated from a $500 \mathrm{~mm}^{3}$ PDX were plated in duplicated at 60.000 cells/well into 8 well-chambers slides. Drugs and vehicle (DMSO) were added after $24 \mathrm{~h}$. To quantify the drug response in PDCs, representative bright field pictures of each well were obtained 7 days post-treatment and normalized against untreated (vehicle). A minimum of three different biological replicates (different tumors) from each model were assayed. For bright field images analysis ImageJ (http://rsb.info.nih.gov/ij/) was used. Two representative areas of single spheroids were quantified individually from at least two independent wells. The mean spheroid area for every treatment was calculated and normalized to untreated controls (vehicles). Relative mean spheroid areas for every treatment condition and the \pm SEM were plotted. 
Cell suspensions generated from a $500 \mathrm{~mm}^{3} \mathrm{PDX}$ were plated in duplicated at 60,000 cells/well into 8 well-chambers slides. After 24 hours, drugs and vehicle (DMSO) as well as $10 \mu \mathrm{M}$ of 8 ethynyl-2'-deoxyuridine (EdU) were added and the cells were incubated for 2 days. EdU staining was performed using the Click-iT ${ }^{\mathrm{TM}}$ EdU Alexa Fluor ${ }^{\mathrm{TM}} 488$ Imaging kit (ThermoFisher Scientific) adapting the manufacturer's instructions. Briefly, the cells were fixed with 3.7\% paraformaldehyde for 15 minutes and permeabilized with $1 \%$ Triton $X-100$ for 20 minutes, all at room temperature. After $1 \mathrm{~h}$ of $5 \%$ BSA in PBS blocking, cells were incubated with the Click-iT ${ }^{\mathrm{TM}}$ reaction cocktail and primary antibodies (mouse Vimentin 1:500 or human CK18 1:100) overnight at room temperature. The following day, cells were washed 3x with 3\% BSA in PBS and incubated with secondary antibodies for 1 hour at room temperature. Finally, cells were washed $3 x$ with $3 \%$ BSA in PBS, mounted with Prolong ${ }^{\mathrm{TM}}$ Antifade Reagent Mountant with DAPI (Molecular Probes) and stored at $-20^{\circ} \mathrm{C}$ until analysis.

Confocal microscopy analysis was carried out using the Nikon confocal microscope $\mathrm{C}^{+}$equipped with LU-N4S laser unit and the NIS-Elements software (Nikon) was used for capturing representative images of spheroids. Number of both DAPI positive and EdU positive cells in each spheroid was obtained using ImageJ (http://rsb.info.nih.gov/ij/). The percentage of Edu positive cells per spheroid was calculated and the mean of every treatment was relativized to the untreated (vehicle). Relative percentage of S-phase entry cells and \pm SEM were plotted.

Molecular modelling of the complex between p16 and P18IN003

We constructed a structural model of the complex between p16 and a known inhibitor P18IN003 using computational methods of docking and molecular dynamics simulations. p16 has four Ankryin repeats and we used the only available apo structure of p16, an NMR structure (PDB: $2 \mathrm{~A} 5 \mathrm{E}$ ), which is very similar to the crystal structure of p16 bound to CDK6 (PDB: 1BI7, root mean squared deviation of $<1 \AA$, confined largely to the loop regions). A homology model ${ }^{62}$ was constructed to model the interactions between p16 and CDK4 based on the p16-CDK6 crystal structure, given that similarity between CDK4 and CDK6 is $81 \%$. This crystal structure was then used to identify the region of interaction between p16 and CDK4 and this region was used to 
define a binding pocket on the surface of the NMR structure of apo p16 (a similar method was used to identify inhibitors of $\mathrm{p} 18^{30}$ ) to which the inhibitor P18IN003 was docked. For docking, the 3D structure of the inhibitor P18IN003 was built using the Maestro module and minimized using the Macromodel module, employing the OPLS-2005 force field, in the program Schrodinger 12.0. The minimized P18IN003 inhibitor was docked into the binding pocket of p16 defined above with the program Glide using standard docking protocols ${ }^{63}$. Out of the top 10 lowest energy poses of the binding of P18IN003 to $\mathrm{p} 16,8$ poses of the inhibitor were very similar to each other and so we chose the top pose and subject the complex to further refinement using molecular dynamics (MD) simulations. The simulations were carried out using the AMBER 18 program, using protocols that we have shown to be successful in previous studies ${ }^{63}$. The partial charges and force field parameters for P18IN003 was generated using the Antechamber module in Amber 18. All atom versions of the amber ff14SB and the general Amber force field (GAFF) were used for the protein and the inhibitors respectively. Simulations were carried out for $100 \mathrm{~ns}$ in triplicates at $300 \mathrm{~K}$ using standard protocols ${ }^{63}$. Simulation trajectories were visualized using VMD and figures were generated using Pymol.

\section{Statistical analysis}

GraphPad Prism version 6.00 for Windows, GraphPad Software, La Jolla California USA (www.graphpad.com) were used for statistical analysis. A bootstrap resampling procedure $(n=$ 2000) was used to calculate the SE in the percentage of change in tumor volume relative to untreated. D'Agostino-Pearson omnibus test was performed to check the normality assumption in all comparative studies. If the null hypothesis of normality was not rejected, we assumed Gaussian distribution of the samples, but if the sample size was too small or the hypothesis was rejected, we did not assume it.

For the comparative experiments of biomarkers between ribociclib and vehicle groups, we used paired $t$-test or Wilcoxon signed-rank test, as appropriate after checking normality assumption. For the comparative between ribociclib-sensitive (S) and -resistant $(R)$ samples, we used an unpaired t-test or Mann-Whitney $\mathrm{U}$ test, as appropriate after checking normality assumption. Adjustment for multiple testing was performed in each biomarker by controlling the false discovery rate at $5 \%$ according Benjamini and Hochberg method. 
Univariate logistic models were used to obtain odds ratios (OR) of studied biomarkers in PDXs

\section{2} and metastatic patient samples with $95 \%$ Cls. To quantify the level of association between a qualitative factor and response we calculated accuracy, sensitivity, specificity, positive and negative predictive values (PPV and NPV). The optimum cut-off points established in this study were selected by the Youden index, which maximizes the sum of the sensitivity and specificity in each biomarker analyzing the ROC curve. Patients were considered sensitive to the treatment in the metastatic setting if a clinical benefit (defined by RECIST criteria) was achieved and maintained for a period $\geq 6$ months and/or $\geq 10$ cycles of treatment.

For patient tumor of ABC-POP trial we performed univariate logistic regressions to estimate the odds ratio for a standard deviation change in continuous $\mathrm{H}$-score biomarkers levels of $\mathrm{p} 16, \mathrm{pRb}$ and cyclin D1 and absolute k67 response. The analysis was performed with R version 4.0.3. (www.R-project.org).

For analyzing the correlation between double genetic hit in $R B 1$ locus and treatment with CDK4/6i, genomic data of metastases from $800 \mathrm{BC}$ was obtained from the Hartwig Medical Foundation $\left(\mathrm{HMF} ;{ }^{34}\right)(\mathrm{DR}-110)$. Patients with 'None', 'Other' or 'Unknown' treatment were filtered out, yielding 582 patients who received known treatments. All metastatic samples bearing single base substitutions and indels causing frameshift variants, stop gained variants, splice acceptor variants, splice donor variants, start lost variants, stop lost variants, missense variants, inframe deletions or inframe insertions affecting the RB1 gene were identified. Metastatic samples with minor and major allele copy number of the genomic region containing the RB1 locus smaller than 0.01 were deemed to carry homozygous deletion of the $R B 1$ gene, while those with minor allele copy number of this genomic region smaller than 0.01 , but greater major allele copy number were deemed to carry a heterozygous deletion of $R B 1$. We used multivariable logistic regression to assess the association between the alteration status of the metastatic breast tumor and the likelihood probability that the patient received CDK4/6i. Two logistic regression formulas modeling different interactions between the variables (mutation and deletion) were applied.

1
3 
815 The authors are grateful to all the patients who kindly consented for the use of their tumors to 816 develop this study and all the personnel involved in sample collection from the Breast Surgical 817 Unit, Breast Cancer Center, and Department of Radiology Vall d'Hebron University Hospital; and 818 Molecular Oncology Group at Vall d'Hebron Institute of Oncology (VHIO). Emmanuelle di Tomaso from Novartis Pharmaceuticals Corporation kindly provided LEE011 and BYL719 for in vivo and ex vivo work. Genentech, Inc. kindly provided GDC-0032 for in vivo and ex vivo work. We are also grateful to Hong Shaoh at the MSKCC, the Molecular Oncology Group (José Jimenez, Lidia Alonso, Lidia Sánchez-Flores and Xavier Guardia) at Vall d'Hebron Institute of Oncology (VHIO) and Molecular Biology Group (Teresa Moline Marimon) at Vall d'Hebron University Hospital for providing technical support with PDXs sequencing and immunostainings. This manuscript was edited by Life Science Editors. ABC-POP trial received funding from Eli-Lilly and from the BCRF. This publication and the underlying research are partly facilitated by Hartwig Medical Foundation and the Center for Personalized Cancer Treatment (CPCT) which have generated, analyzed and made available data for this research. 
8451 Hortobagyi, G. N. et al. Ribociclib as First-Line Therapy for HR-Positive, Advanced Breast Cancer. $846 \quad$ N Engl J Med 375, 1738-1748, doi:10.1056/NEJMoa1609709 (2016).

8472 Finn, R. S. et al. Palbociclib and Letrozole in Advanced Breast Cancer. N Engl J Med 375, 1925$848 \quad$ 1936, doi:10.1056/NEJMoa1607303 (2016).

8493 Goetz, M. P. et al. MONARCH 3: Abemaciclib As Initial Therapy for Advanced Breast Cancer. J $850 \quad$ Clin Oncol 35, 3638-3646, doi:10.1200/JCO.2017.75.6155 (2017).

8514 Dickler, M. N. et al. MONARCH 1, A Phase II Study of Abemaciclib, a CDK4 and CDK6 Inhibitor, 852 as a Single Agent, in Patients with Refractory HR(+)/HER2(-) Metastatic Breast Cancer. Clin 853 Cancer Res 23, 5218-5224, doi:10.1158/1078-0432.CCR-17-0754 (2017).

8545 Johnston, S. R. D. et al. Abemaciclib Combined With Endocrine Therapy for the Adjuvant Treatment 855 of HR+, HER2-, Node-Positive, High-Risk, Early Breast Cancer (monarchE). J Clin Oncol 38, 3987$856 \quad 3998$, doi:10.1200/JCO.20.02514 (2020).

8576 Tolaney, S. M. et al. Abemaciclib plus trastuzumab with or without fulvestrant versus trastuzumab 858 plus standard-of-care chemotherapy in women with hormone receptor-positive, HER2-positive 859 advanced breast cancer (monarcHER): a randomised, open-label, phase 2 trial. Lancet Oncol, 860 doi:10.1016/S1470-2045(20)30112-1 (2020).

8617 Ciruelos, E. et al. Palbociclib and Trastuzumab in HER2-Positive Advanced Breast Cancer: Results 862 from the Phase II SOLTI-1303 PATRICIA Trial. Clin Cancer Res 26, 5820-5829, doi:10.1158/1078$863 \quad$ 0432.CCR-20-0844 (2020).

8648 Herrera-Abreu, M. T. et al. Early Adaptation and Acquired Resistance to CDK4/6 Inhibition in 865 Estrogen Receptor-Positive Breast Cancer. Cancer Res 76, 2301-2313, doi:10.1158/00085472.CAN-15-0728 (2016).

8679 Formisano, L. et al. Aberrant FGFR signaling mediates resistance to CDK4/6 inhibitors in ER+ breast cancer. Nat Commun 10, 1373, doi:10.1038/s41467-019-09068-2 (2019).

86910 Costa, C. et al. PTEN Loss Mediates Clinical Cross-Resistance to CDK4/6 and PI3Kalpha Inhibitors in Breast Cancer. Cancer Discov 10, 72-85, doi:10.1158/2159-8290.CD-18-0830 (2020). Jansen, V. M. et al. Kinome-Wide RNA Interference Screen Reveals a Role for PDK1 in Acquired Resistance to CDK4/6 Inhibition in ER-Positive Breast Cancer. Cancer Res 77, 2488-2499, doi:10.1158/0008-5472.CAN-16-2653 (2017).

$87412 \quad$ Yang, C. et al. Acquired CDK6 amplification promotes breast cancer resistance to CDK4/6 inhibitors and loss of ER signaling and dependence. Oncogene 36, 2255-2264, doi:10.1038/onc.2016.379 (2017). 
Li, Z. et al. Loss of the FAT1 Tumor Suppressor Promotes Resistance to CDK4/6 Inhibitors via the Hippo Pathway. Cancer Cell 34, 893-905 e898, doi:10.1016/j.ccell.2018.11.006 (2018). O'Leary, B. et al. The Genetic Landscape and Clonal Evolution of Breast Cancer Resistance to Palbociclib plus Fulvestrant in the PALOMA-3 Trial. Cancer discovery 8, 1390-1403, doi:10.1158/2159-8290.CD-18-0264 (2018).

883 Condorelli, R. et al. Polyclonal RB1 mutations and acquired resistance to CDK 4/6 inhibitors in patients with metastatic breast cancer. Ann Oncol 29, 640-645, doi:10.1093/annonc/mdx784 (2018). Turner, N. C. et al. Cyclin E1 Expression and Palbociclib Efficacy in Previously Treated Hormone Receptor-Positive Metastatic Breast Cancer. J Clin Oncol 37, 1169-1178, doi:10.1200/JCO.18.00925 (2019). Andre, F. et al. Alpelisib for PIK3CA-Mutated, Hormone Receptor-Positive Advanced Breast Cancer. N Engl J Med 380, 1929-1940, doi:10.1056/NEJMoa1813904 (2019).

891 Gao, H. et al. High-throughput screening using patient-derived tumor xenografts to predict clinical trial drug response. Nat Med 21, 1318-1325, doi:10.1038/nm.3954 (2015). Bruna, A. et al. A Biobank of Breast Cancer Explants with Preserved Intra-tumor Heterogeneity to Screen Anticancer Compounds. Cell 167, 260-274 e222, doi:10.1016/j.cell.2016.08.041 (2016). Therasse, P. et al. New guidelines to evaluate the response to treatment in solid tumors. European Organization for Research and Treatment of Cancer, National Cancer Institute of the United States, National Cancer Institute of Canada. J Natl Cancer Inst 92, 205-216, doi:10.1093/jnci/92.3.205 (2000).

Cejalvo, J. M. et al. Intrinsic Subtypes and Gene Expression Profiles in Primary and Metastatic Breast Cancer. Cancer Res 77, 2213-2221, doi:10.1158/0008-5472.CAN-16-2717 (2017).

902 Finn, R. S. et al. PD 0332991, a selective cyclin D kinase 4/6 inhibitor, preferentially inhibits proliferation of luminal estrogen receptor-positive human breast cancer cell lines in vitro. Breast Cancer Res 11, R77, doi:10.1186/bcr2419 (2009).

Cheng, D. T. et al. Memorial Sloan Kettering-Integrated Mutation Profiling of Actionable Cancer Targets (MSK-IMPACT): A Hybridization Capture-Based Next-Generation Sequencing Clinical Assay for Solid Tumor Molecular Oncology. J Mol Diagn 17, 251-264, doi:10.1016/j.jmoldx.2014.12.006 (2015).

O'Leary, B. et al. Circulating tumor DNA markers for early progression on fulvestrant with or without palbociclib in ER+ advanced breast cancer. Journal of the National Cancer Institute Article in press, doi:doi.org/10.1093/jnci/djaa087 (2020). 

and cell lines. Cancer Res 55, 505-509 (1995).

$91226 \quad$ Lukas, J. et al. Retinoblastoma-protein-dependent cell-cycle inhibition by the tumour suppressor p16. Nature 375, 503-506, doi:10.1038/375503a0 (1995). E6/E7 gene expression in head and neck cancers with unaltered p53 status and perturbed pRb cell cycle control. Oncogene 21, 1510-1517, doi:10.1038/sj.onc.1205214 (2002). Lu, D. W., El-Mofty, S. K. \& Wang, H. L. Expression of p16, Rb, and p53 proteins in squamous cell carcinomas of the anorectal region harboring human papillomavirus DNA. Mod Pathol 16, 692-699, doi:10.1097/01.MP.0000077417.08371.CE (2003).

921

Arnedos, M. et al. Modulation of Rb phosphorylation and antiproliferative response to palbociclib: doi:10.1093/annonc/mdy202 (2018).

Gao, Y. et al. Small-molecule inhibitors targeting INK4 protein p18(INK4C) enhance ex vivo expansion of haematopoietic stem cells. Nat Commun 6, 6328, doi:10.1038/ncomms7328 (2015). Green, J. L. et al. Direct CDKN2 Modulation of CDK4 Alters Target Engagement of CDK4 Inhibitor Drugs. Mol Cancer Ther 18, 771-779, doi:10.1158/1535-7163.MCT-18-0755 (2019). Arnedos, M. et al. Randomized preoperative window of opportinity (WOO) study with the CDK4/6 inhibitor abemaciclib in early breast cancer (EBC) patients and differential gene expression pathway analysis with palbociclib Ann Oncol, doi:10.1016/annonc/annonc267 (2020). cancer with anastrozole or tamoxifen alone or combined correlate with recurrence-free survival. Clin Cancer Res 11, 951s-958s (2005). Priestley, P. et al. Pan-cancer whole-genome analyses of metastatic solid tumours. Nature 575, 210-216, doi:10.1038/s41586-019-1689-y (2019).

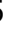
Bosch, A. et al. PI3K inhibition results in enhanced estrogen receptor function and dependence in hormone receptor-positive breast cancer. Sci Transl Med 7, 283ra251, doi:10.1126/scitransImed.aaa4442 (2015).

Rugo, H. S. et al. BYLieve: A phase II study of alpelisib (ALP) with fulvestrant (FUL) or letrozole (LET) for treatment of PIK3CA mutant, hormone receptor-positive $(\mathrm{HR}+)$, human epidermal growth factor receptor 2-negative (HER2-) advanced breast cancer (aBC) progressing on/after cyclindependent kinase 4/6 inhibitor (CDK4/6i) therapy. Journal of Clinical Oncology 36, TPS1107TPS1107, doi:10.1200/JCO.2018.36.15_suppl.TPS1107 (2018). 

Engl J Med 379, 1926-1936, doi:10.1056/NEJMoa1810527 (2018).

94538 Rossi, L. et al. Clinical outcomes after palbociclib with or without endocrine therapy in postmenopausal women with hormone receptor positive and HER2-negative metastatic breast cancer enrolled in the TREnd trial. Breast Cancer Res 21, 71, doi:10.1186/s13058-019-1149-5 (2019). in Hormone Receptor-Positive, ERBB2-Negative Breast Cancer-Reply. JAMA Oncol, doi:10.1001/jamaoncol.2020.1518 (2020). Chandarlapaty, S. et al. Prevalence of ESR1 Mutations in Cell-Free DNA and Outcomes in Metastatic Breast Cancer: A Secondary Analysis of the BOLERO-2 Clinical Trial. JAMA Oncol 2, 1310-1315, doi:10.1001/jamaoncol.2016.1279 (2016).

Malorni, L. et al. Palbociclib as single agent or in combination with the endocrine therapy received before disease progression for estrogen receptor-positive, HER2-negative metastatic breast cancer: TREnd trial. Ann Oncol 29, 1748-1754, doi:10.1093/annonc/mdy214 (2018). DeMichele, A. et al. CDK 4/6 inhibitor palbociclib (PD0332991) in Rb+ advanced breast cancer: phase II activity, safety, and predictive biomarker assessment. Clin Cancer Res 21, 995-1001, doi:10.1158/1078-0432.CCR-14-2258 (2015).

Patnaik, A. et al. Efficacy and Safety of Abemaciclib, an Inhibitor of CDK4 and CDK6, for Patients with Breast Cancer, Non-Small Cell Lung Cancer, and Other Solid Tumors. Cancer Discov 6, 740753, doi:10.1158/2159-8290.CD-16-0095 (2016). palbociclib inhibition. Science 366, doi:10.1126/science.aaw2106 (2019). Li, P., Zhang, X., Gu, L., Zhou, J. \& Deng, D. P16 methylation increases the sensitivity of cancer cells to the CDK4/6 inhibitor palbociclib. PLoS One 14, e0223084, doi:10.1371/journal.pone.0223084 (2019). De Laurentiis, M. et al. BioltaLEE-Biomarker analysis on lquid biopsies of patientes treated with ribociclib and letrozole as first-line therapy foradvanced breast cancer (ABC) San Antonio Breast Cancer Sympsium P5-01-07 (2019). Wander, S. A. et al. The genomic landscape of intrinsic and acquired resistance to cyclin-dependent kinase 4/6 inhibitors in patients with hormone receptor positive metastatic breast cancer. Cancer Discov, doi:10.1158/2159-8290.CD-19-1390 (2020). 

Suppressor Gene. Cancer Discov 9, 248-263, doi:10.1158/2159-8290.CD-18-0469 (2019).

$97950 \quad$ Gelbert, L. M. et al. Preclinical characterization of the CDK4/6 inhibitor LY2835219: in-vivo cell cycle-dependent/independent anti-tumor activities alone/in combination with gemcitabine. Invest New Drugs 32, 825-837, doi:10.1007/s10637-014-0120-7 (2014). Gris-Oliver, A. et al. Genetic Alterations in the PI3K/AKT Pathway and Baseline AKT Activity Define AKT Inhibitor Sensitivity in Breast Cancer Patient-derived Xenografts. Clin Cancer Res, doi:10.1158/1078-0432.CCR-19-3324 (2020). Vora, S. R. et al. CDK 4/6 inhibitors sensitize PIK3CA mutant breast cancer to PI3K inhibitors. Cancer Cell 26, 136-149, doi:10.1016/j.ccr.2014.05.020 (2014). pathways from the PALOMA-2 study. Cancer Res Abstract P2-09-10 (2018). Ma, C. X. et al. NeoPalAna: Neoadjuvant Palbociclib, a Cyclin-Dependent Kinase 4/6 Inhibitor, and Anastrozole for Clinical Stage 2 or 3 Estrogen Receptor-Positive Breast Cancer. Clin Cancer Res 23, 4055-4065, doi:10.1158/1078-0432.CCR-16-3206 (2017). Ciruelos, E. et al. SOLTI-1303 PATRICIA phase II trial (STAGE 1) -- Palbociclib and trastuzumab in postmenopausal patients with HER2-positive metastatic breast cancer. Cancer Res Abstract PD3-03 (2019). Tentler, J. J. et al. Patient-derived tumour xenografts as models for oncology drug development. Nat Rev Clin Oncol 9, 338-350, doi:10.1038/nrclinonc.2012.61 (2012). Eyre, R. et al. Patient-derived Mammosphere and Xenograft Tumour Initiation Correlates with Progression to Metastasis. J Mammary Gland Biol Neoplasia 21, 99-109, doi:10.1007/s10911-0169361-8 (2016).

1004 Perou, C. M. et al. Molecular portraits of human breast tumours. Nature 406, 747-752, doi:10.1038/35021093 (2000). clinical implications. Proc Natl Acad Sci U S A 98, 10869-10874, doi:10.1073/pnas.191367098 (2001).

Lin, K. et al. An ATP-site on-off switch that restricts phosphatase accessibility of Akt. Sci Signal 5, ra37, doi:10.1126/scisignal.2002618 (2012).

Vandesompele, J. et al. Accurate normalization of real-time quantitative RT-PCR data by geometric averaging of multiple internal control genes. Genome Biol 3, RESEARCH0034, doi:10.1186/gb2002-3-7-research0034 (2002). 

Biol 234, 779-815, doi:10.1006/jmbi.1993.1626 (1993).

101263 Kannan, S. et al. Probing the binding mechanism of Mnk inhibitors by docking and molecular dynamics simulations. Biochemistry 54, 32-46, doi:10.1021/bi501261j (2015).

1014

1015

1016

1017

1018

1019

1020

1021

1022

1023

1024

1025

1026

1027

1028

1029

1030

1031

1032

1033

1034

1035

1036

1037

1038

1039

1040

1041

1042

1043 
Figure 1

A

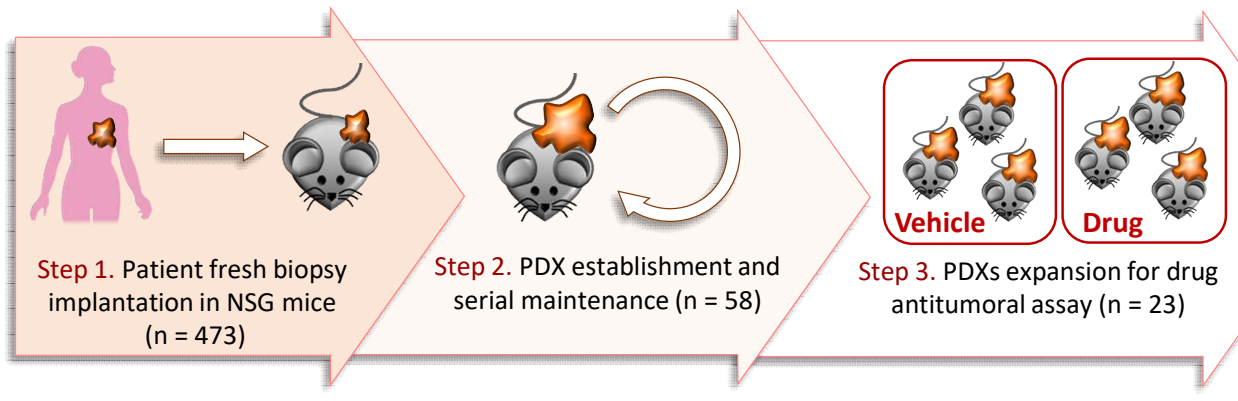

B

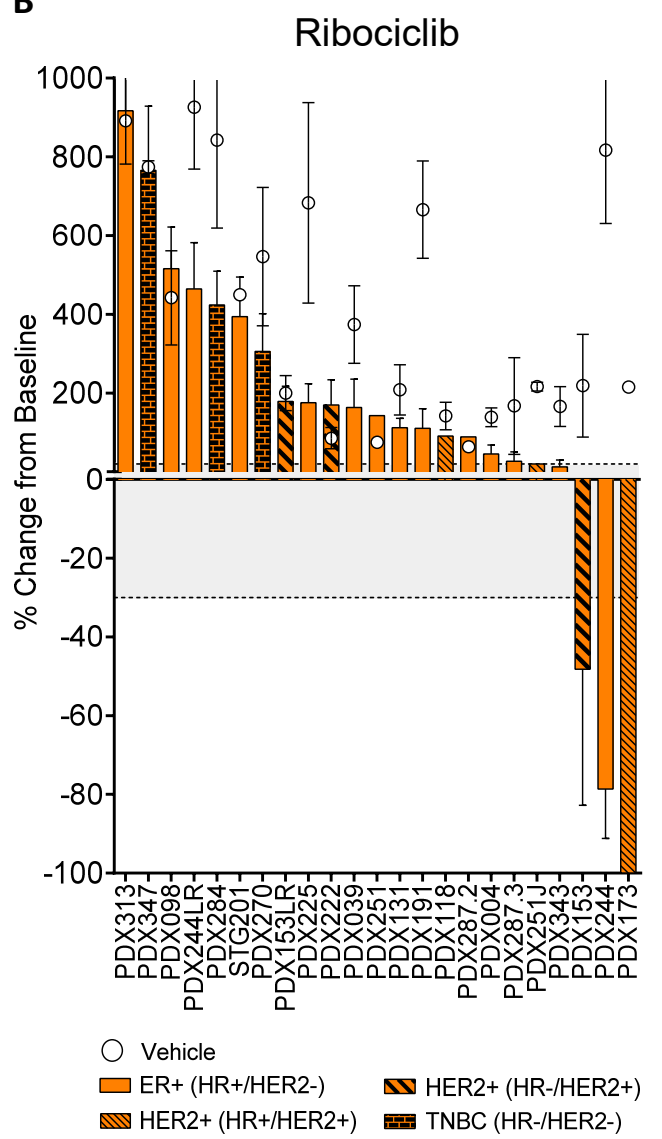

C

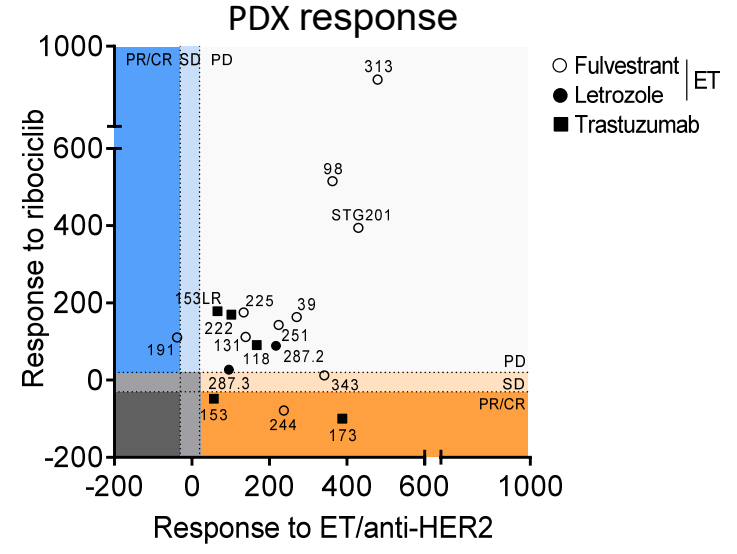

D

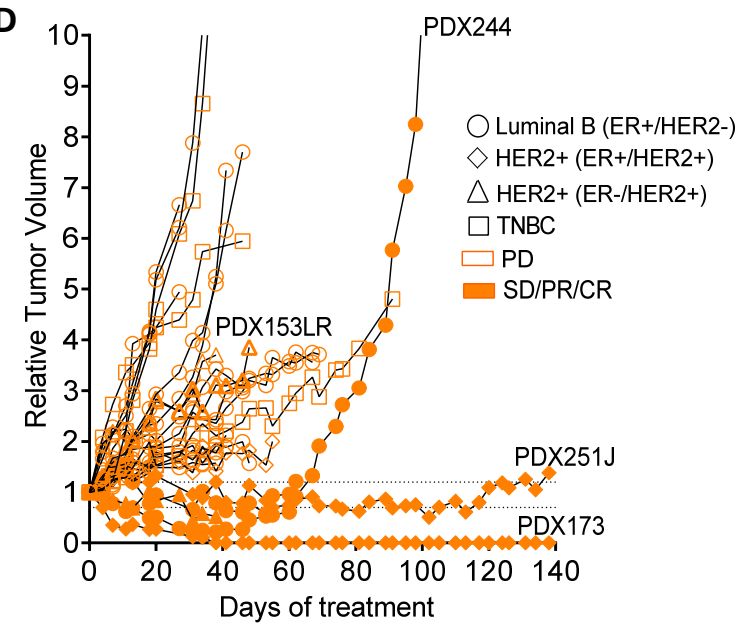

Figure 1. Ribociclib monotherapy has higher antitumor activity than other targeted agents in $\mathrm{ER}^{+}$and HER2 ${ }^{+}$BC PDXs. A) Workflow depicting the generation of BC PDX models from BC patient samples and its subsequent expansion for targeted treatment screening. B) Waterfall plot representing the growth of 23 PDX treated with ribociclib $75 \mathrm{mg} / \mathrm{kg}$ (bars) and vehicle (circles). The percentage change from the initial volume is shown at day 35 of treatment. Dashed lines indicate the range of PD (>20\%), SD (20\% to $-30 \%)$ and PR/CR (<-30\%). The molecular subtypes are indicated. Data represent means and error bars \pm SEM. C) Antitumor response of ribociclib 
1053 (y-axis) vs. other targeted agents (x-axis; endocrine therapy or trastuzumab) in PDXs represented 1054 as the percentage of tumor volume change compared to the initial tumor volume. Symbol shapes 1055 represent the different targeted therapies. D) Spaghetti plot showing the relative tumor volume 1056 change along time in 23 BC PDX treated with ribociclib $75 \mathrm{mg} / \mathrm{kg}$. Ribociclib-sensitive models are 1057 represented with fill symbols and ribociclib-resistant with empty symbols. Symbol shapes represent the PDX's molecular subtypes. Dashed lines indicate the range of PD (>1.2), SD (1.2 1059 to -0.7) and PR/CR (<-0.7). Acquisition of ribociclib resistance in PDX244 (PDX244LR) is shown. 1060

1061

1062

1063

1064

1065

1066

1067

1068

1069

1070

1071

1072

1073

1074

1075

1076

1077 


\section{Figure 2}

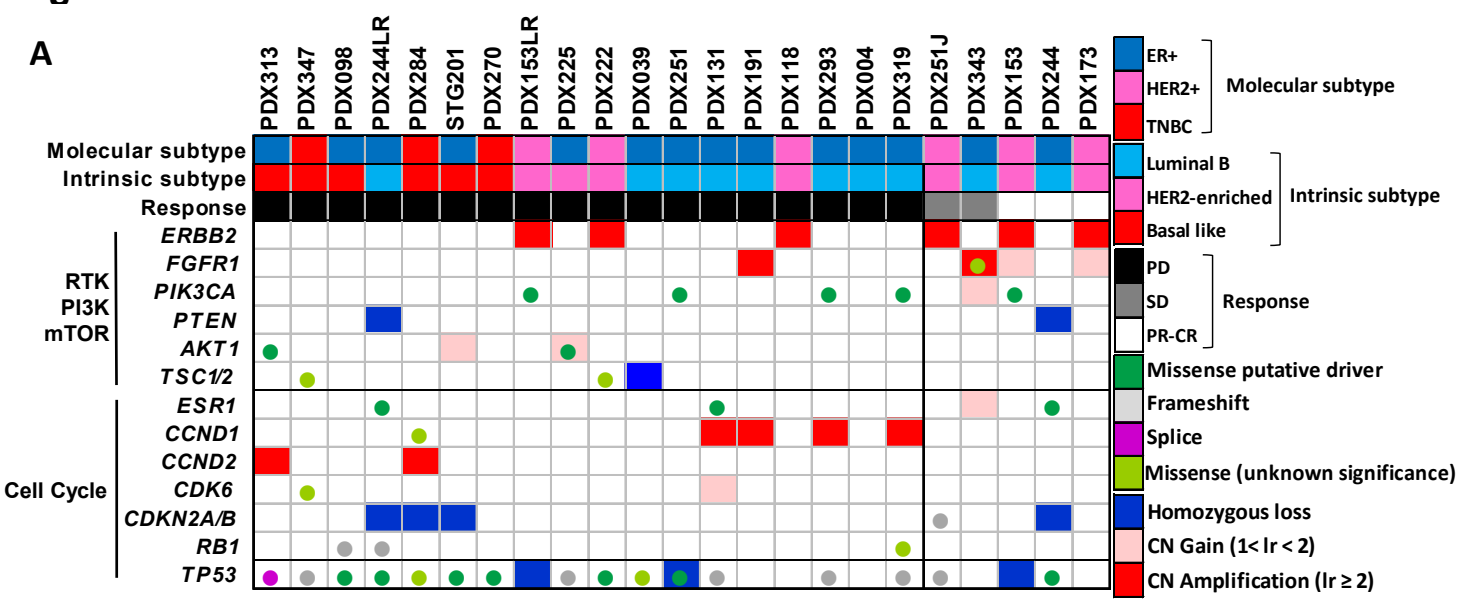

B

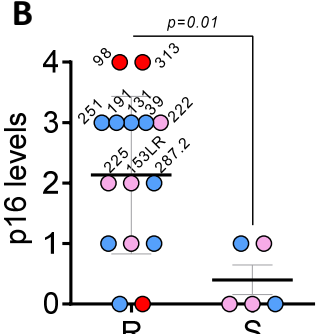

$\mathrm{R}$

$\mathrm{S}$
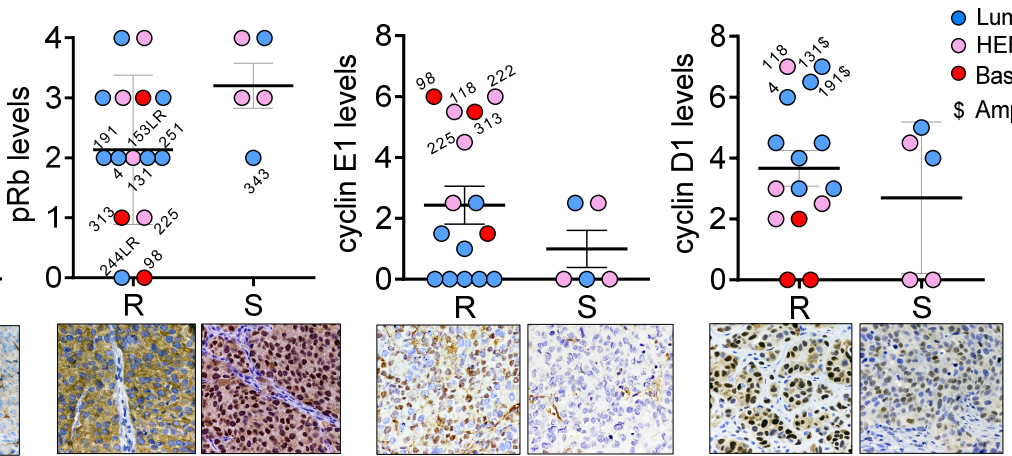

C

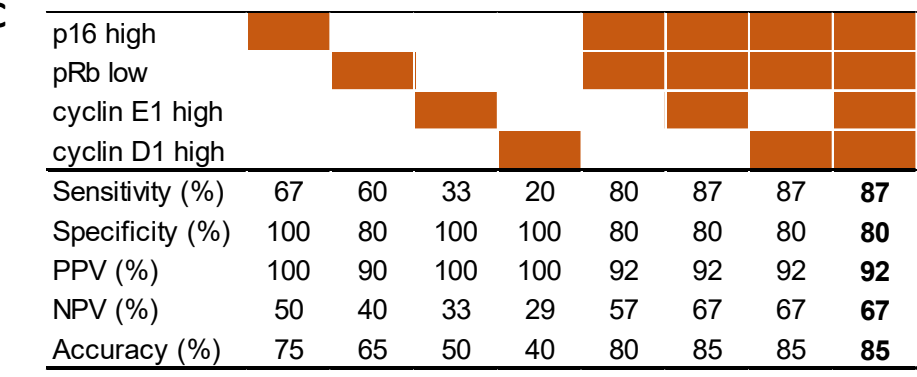

D
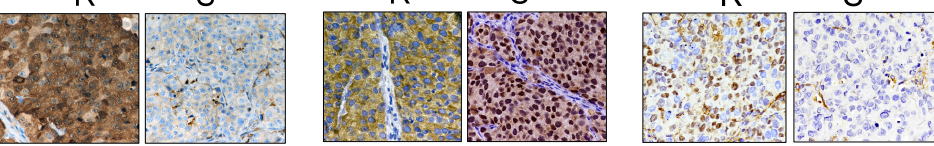

E

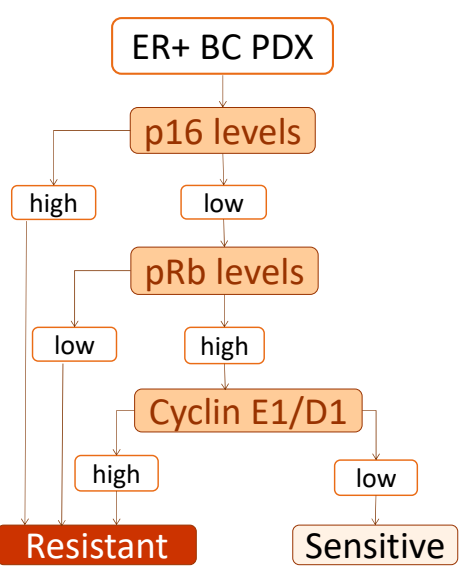

\begin{tabular}{|c|c|c|c|}
\hline \multicolumn{2}{|c|}{ Protein } & \multicolumn{2}{c|}{ mRNA } \\
\hline Log2 OR & $\boldsymbol{p}$-value & Log2 OR & $\boldsymbol{p}$-value \\
\hline 2.8 & 0.006 & $>3$ & $<0.001$ \\
2.9 & 0.02 & $>3$ & $<0.001$ \\
$<-3$ & 0.8 & $>3$ & $<0.001$ \\
\hline
\end{tabular}

Figure 2. PDXs expressing high p16 are resistant to ribociclib. A) Summary of genetic alterations in the PDX panel from Figure 1B, including the PDX subtype classification, based on IHC (Molecular subtype) or PAM50 analysis (intrinsic subtype), and the response to CDK4/6 inhibitors. Genes with similar function such as TSC1/TSC2 or CDKN2A/CDKN2B were considered as one single feature. B) Quantification of IHC staining for p16, pRb, cyclin E1 and cyclin D1 in 23-untreated PDX in relationship with ribociclib-response. Semiquantitative analysis was performed for $\mathrm{pRb}$ and $\mathrm{p} 16$, or the Allred scoring method for cyclin $\mathrm{E} 1$ and cyclin $\mathrm{D} 1$ in 
relationship with ribociclib-response. Different colors indicate the PDX intrinsic subtype and \$ indicates the models harboring gene amplification. Mean and \pm SEM are indicated. $p$-value, unpaired parametric $t$-test. The pictures underneath are representative bright field images of high/low staining of each protein. Magnification 40x. R: resistant; S: sensitive. C) Prediction analysis of the indicated biomarker(s) to classify a PDX as resistant or sensitive to ribociclib based on their expression levels and according to Youden index. High p16 means expression score $\geq$ $2+$, low pRb means expression score $\leq 1+$ and high cyclin E1/D1 means Allred score $>4 / 6$. D) Co-occurrence of altered p16, pRb and cyclin E1 expression levels in a cohort of $814 \mathrm{ER}^{+}$breast invasive carcinomas from the TCGA database using cBioportal (www.cBioportal.org). The cut-off for high versus low protein/mRNA levels is indicated. OR: odd's ratio; prot: protein; SD: standard deviation. E) Consort flow diagram for classifying the PDX responses to ribociclib based on the molecular subtype, p16, pRb and cyclin E1/D1 scores.

.


Figure 3

A

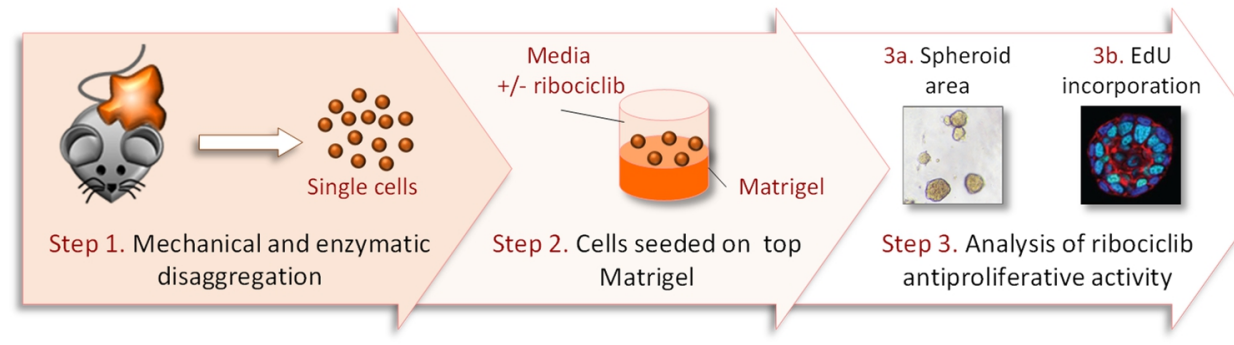

B
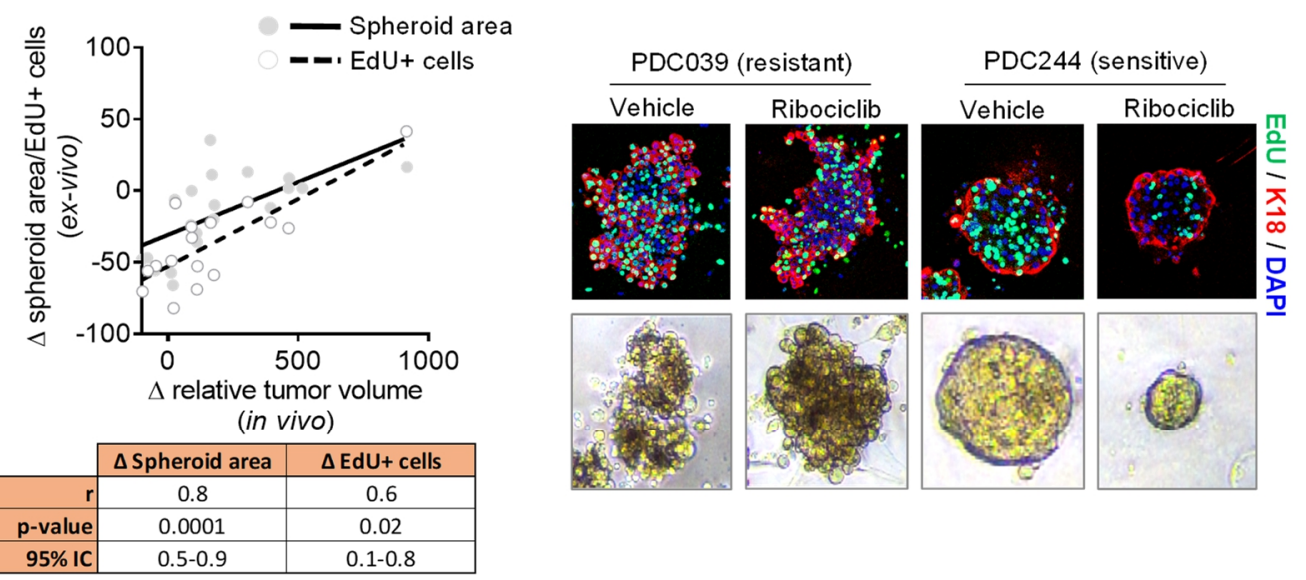

C

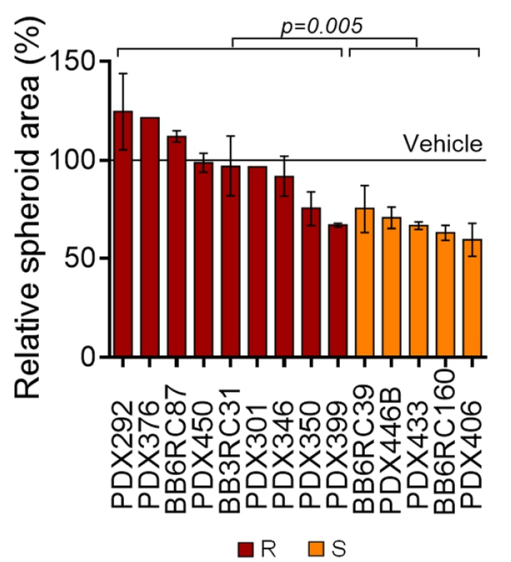

D

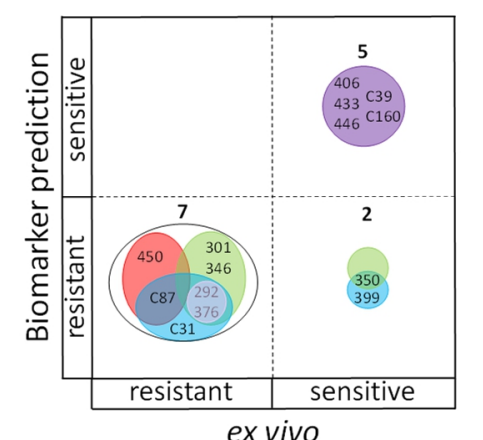

ex vivo

p16 high $\square$ pRb low

Cyclin E1 high $\square$ Cyclin D1 high $\square$ pRb high, p16 low and Cyclin D1 low
$\mathbf{E}$

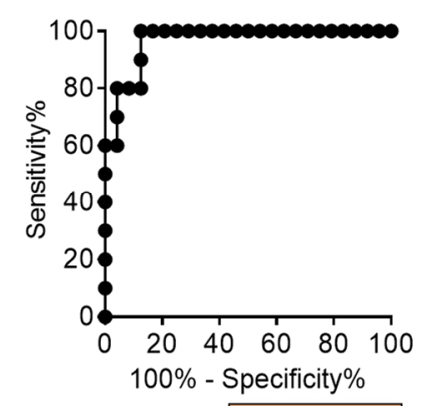

\begin{tabular}{|r|c|}
\cline { 2 - 2 } \multicolumn{1}{c|}{} & $\Delta$ Spheroid area \\
\hline cut-off (\%) & -25 \\
\hline p-value & $<0.0001$ \\
\hline sensitivity (\%) & 100 \\
\hline $95 \%$ IC & $69.2-100$ \\
\hline specificity (\%) & 87.5 \\
\hline $95 \%$ IC & $67.6-97.3$ \\
\hline
\end{tabular}

1112 Figure 3. Biomarker validation in short-term patient-derived tumor cells (PDCs). A)

1113 Workflow depicting the generation of BC PDCs short-term ex vivo cultures from PDXs and the

1114 subsequent analysis of ribociclib response using two different read-outs. B) Correlation analysis

1115 of the ex vivo response of PDCs (y-axis) vs. the in vivo response of the corresponding PDXs ( $\mathrm{x}-$

1116 axis), measured as spheroid area change (empty dots) or the change in EdU incorporation (filled

1117 dots) after ribociclib treatment vs. the change in the relative tumor volume. The Spearman's

1118 coefficient $(r)$, p-value and $95 \%$ of confidence interval $(95 \% \mathrm{Cl})$ for each read-out are summarized

1119 below the graph. Representative images of one ribociclib-resistant (PDC039) and one ribociclib- 
sensitive (PDC244) model treated with vehicle or ribociclib are shown on the right panel, namely

1121

1122

1123

1124

1125

1126

1127

1128

1129

1130

1131

1132

1133

1134

1135

1136

1137

1138

1139

1140

1141

1142

1143

EdU/K18 staining by confocal microscopy and bright field. EdU is shown in green, cytokeratin 18 (K18) in red and DAPI in blue. Magnification 40x. C) Relative spheroid area in 14 PDC models classified as resistant (maroon) or sensitive (orange) according to the composite biomarker. Treatment with $1 \mu \mathrm{M}$ ribociclib for 7 days. Relative data to the vehicle control (100\%) is represented as mean of three independent experiments \pm SEM. $p$-value, unpaired parametric $t$ test. D) Concordance analysis of PDXs' responses to ribociclib based on biomarker prediction ( $\mathrm{y}$ axis) vs. the ex vivo response (x-axis). Biomarkers are represented by circles with different colors and the number of PDX within each category is indicated. E) ROC-curve of the spheroid area increment for ribociclib response prediction in 37 BC PDCs. The p-values and the $95 \%$ confidence interval $(95 \% \mathrm{Cl})$ are summarized below the graph.

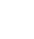

.




\section{Figure 4}

A

\begin{tabular}{|c|c|c|c|c|}
\hline \multicolumn{2}{|c|}{$\begin{array}{l}\text { Treatment } \\
\text { Cell lines }\end{array}$} & \multirow{2}{*}{$\begin{array}{c}\begin{array}{c}\text { Fulvestrant } \\
\mathrm{nM}(\mathrm{fc})\end{array} \\
0.2\end{array}$} & \multirow{2}{*}{$\begin{array}{c}\begin{array}{c}\text { Ribociclib } \\
\mu \mathrm{M}(\mathrm{fc})\end{array} \\
2.2\end{array}$} & \multirow{2}{*}{$\begin{array}{l}\text { Combination } \\
\mathrm{nM}: \mu \mathrm{M}(\mathrm{fc})\end{array}$} \\
\hline & MOCK & & & \\
\hline T47D & p16 & $0.9(4.5)$ & $44.9(20.4)$ & $1.8(6)$ \\
\hline
\end{tabular}
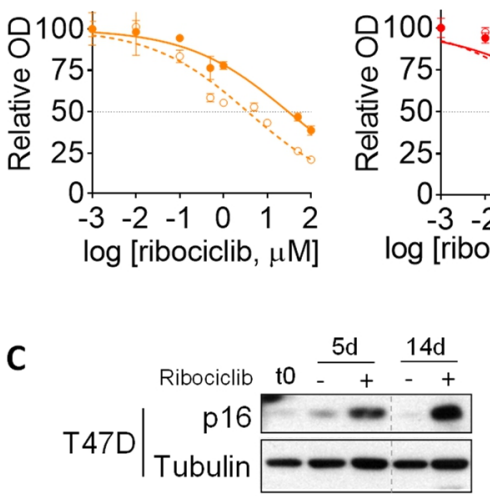

E

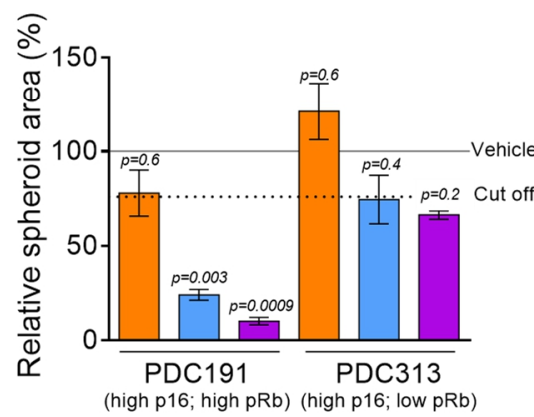

$\square$ Ribociclib $\square$ P18N003 $\square$ P18N003 + Ribociclib

G

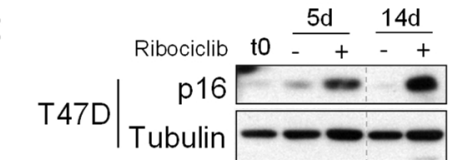
$(\mu \mathrm{M} ; \mathrm{nM})$

\section{D}

B

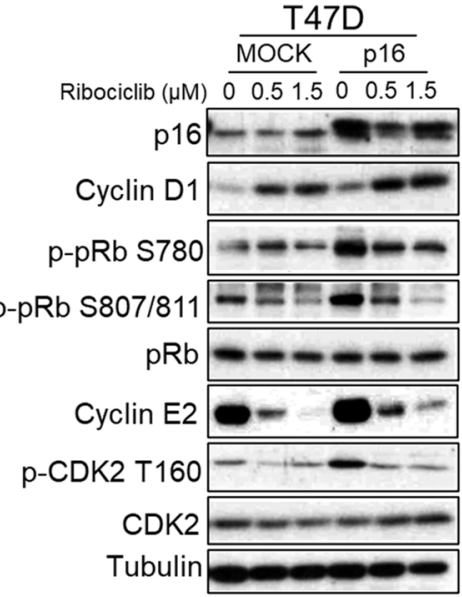

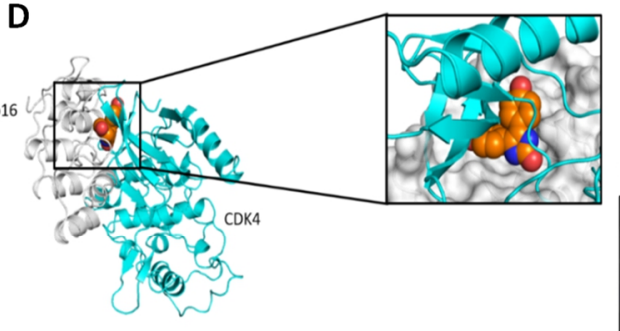

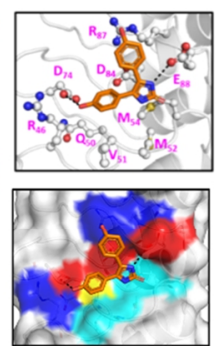

F

\begin{tabular}{|l|l|c|c|c|}
\hline \multicolumn{2}{|c|}{ Treatment } & $\begin{array}{c}\text { Fulvestrant } \\
\mathrm{nM}(\mathrm{fc})\end{array}$ & $\begin{array}{c}\text { Ribociclib } \\
\mu \mathrm{M}(\mathrm{fc})\end{array}$ & $\begin{array}{c}\text { Combination } \\
\mathrm{nM}: \mu \mathrm{M}(\mathrm{fc})\end{array}$ \\
\hline \multirow{2}{*}{ T47D } & MOCK & $\mathbf{0 . 5}$ & 4.4 & 0.1 \\
\cline { 2 - 5 } & cyclin D1 & $2(4.0)$ & $13.7(3.1)$ & $0.5(5.0)$ \\
\hline
\end{tabular}
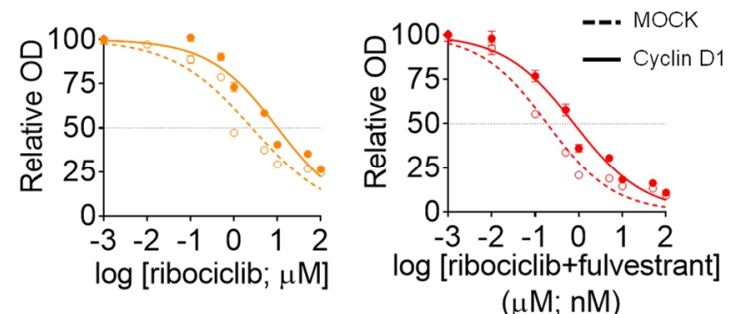

H

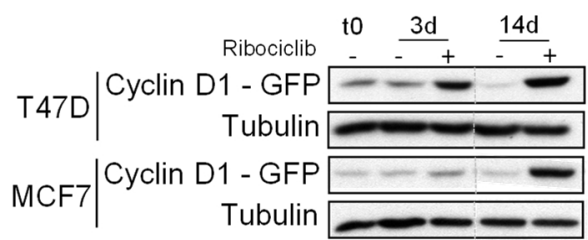

Figure 4. p16 and cyclin D1 overexpression attenuate the response to ribociclib in $\mathrm{ER}^{+} \mathrm{BC}$

cell lines. A) Half-maximal inhibitory concentration $\left(\mathrm{IC}_{50}\right)$ values of ribociclib, fulvestrant and the combination of T47D cells overexpressing p16 (CDKN2A) and fold-change values (fc; in brackets) compared to controls (MOCK), evaluated after 6-days dose-response experiments as shown underneath. At least three independent experiments were conducted with three technical replicates per condition. B) Immunoblot of the indicated proteins in MOCK and p16 overexpressing T47D cells untreated or treated with ribociclib for 5 days at the indicated 
concentrations. C) Immunoblot of the indicated proteins in an enrichment assay. D) Comparison of structural models built in this study for the complexes of p16 bound to P18IN003 and p16

1154 bound to CDK4, showing that CDK4 and P18IN003 share the same binding pocket on p16 and hence CDK4 cannot bind to $\mathrm{p} 16$ when P18IN003 is bound to $\mathrm{p} 16$ (grey cartoon is $\mathrm{p} 16$, orange spheres represent P18IN003, cyan cartoon is CDK4); upper zoomed view is the binding pocket with p16 shown as grey surface and lower zoomed views are the same, highlighting the residues in the binding pocket of p16 as sticks and the hydrogen bonds made between P18IN003 and p16 shown as black dashed lines. E) Relative spheroid area of PDC191 and PDC313 after treatment with $1 \mu \mathrm{M}$ ribociclib, 20nM P18IN003 and the combination in ex vivo cultures for 7 days. Data are presented as means of three independent experiments \pm SEM. $p$-values are based on the oneway ANOVA test with Tukey's method correction compared with the vehicle (black line). Dashed line indicates the optimal cut-off established in Figure 3E. p16 and pRb scores of each PDC are indicated. F) Half-maximal inhibitory concentration $\left(\mathrm{IC}_{50}\right)$ values of ribociclib, fulvestrant and the combination of T47D cells overexpressing cyclin D1 (CCND1) and fold-change values (fc; in brackets) compared to controls (MOCK), evaluated after 6-days dose-response experiments as shown underneath. At least three independent experiments were conducted with three technical replicates. G) Immunoblot of indicated proteins in control (MOCK) and cyclin D1 (CCND1) overexpressing T47D (left panel) and MCF7 (right panel) cells untreated or treated with $0.5 \mu \mathrm{M}$ ribociclib for 48 hours. H) Immunoblot of the indicated proteins in an enrichment experiment. 
Figure 5

CDK4/6i in neoadjuvant setting $B C(n=72)$

A

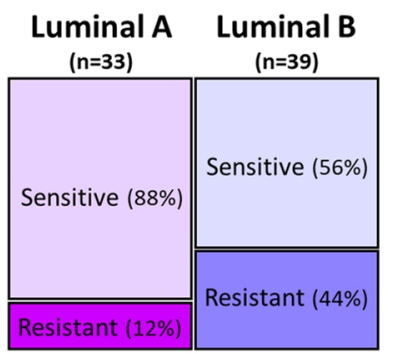

C

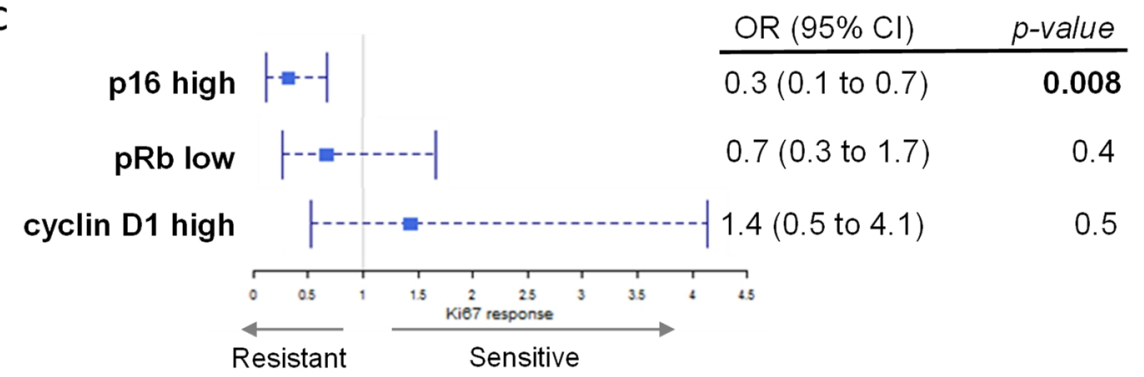

D
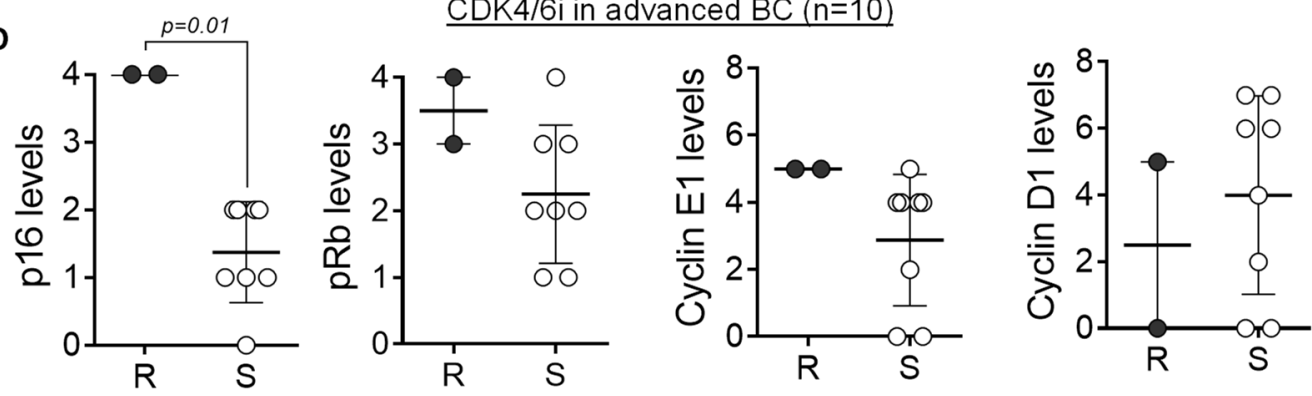

E
B
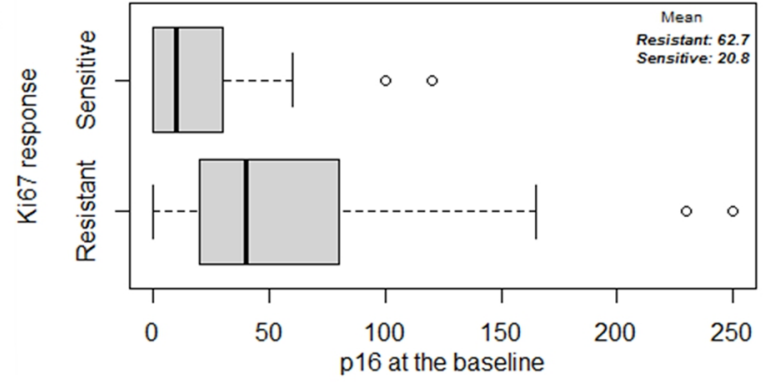

\begin{tabular}{lr} 
OR $(95 \% \mathrm{Cl})$ & $p$-value \\
\hline $0.3(0.1$ to 0.7$)$ & 0.008 \\
$0.7(0.3$ to 1.7$)$ & 0.4 \\
$1.4(0.5$ to 4.1$)$ & 0.5
\end{tabular}

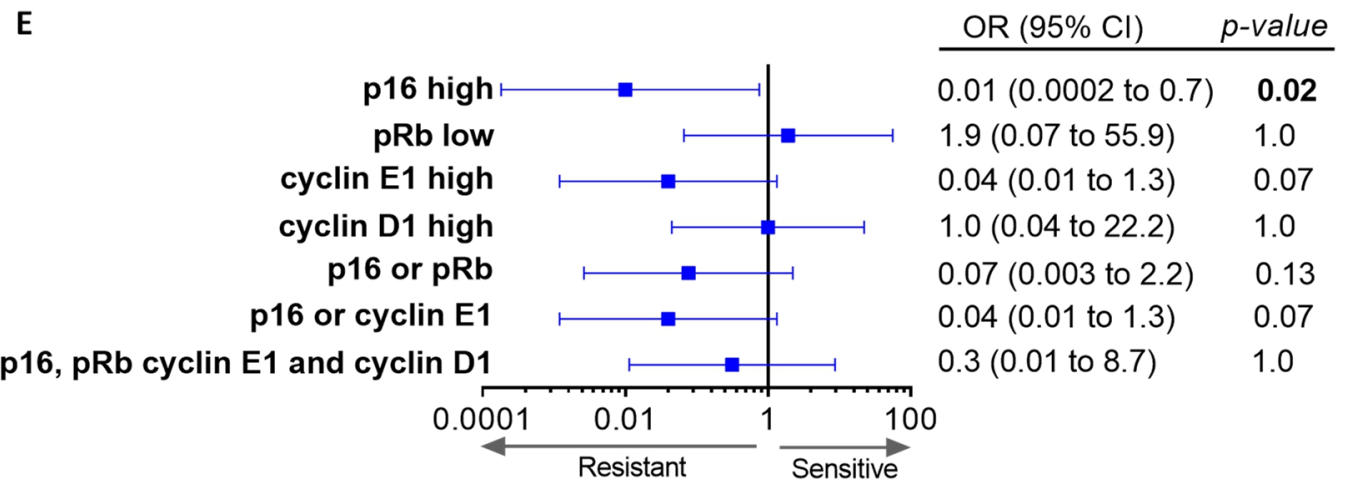

Figure 5. High p16 levels associated with lack of response to CDK4/6i in $E R^{+} B C$ patients.

A) Representation of the percentage distribution of Luminal $A / B$ tumors vs. sensitivity to abemaciclib after 15 days of treatment in the neoadjuvant setting in the ABC-POP trial. Tumors were classified as Luminal A if $\%$ Ki67 $<15$ or as Luminal B if $\%$ Ki67 $\geq 15$. Tumors showing In Ki67 $<1$ at day 15 were considered sensitive and those with In Ki67 $\geq 1$ were resistant to the studied drug. B) Logistic model to evaluate the effect of p16 on the response to abemaciclib. Mean value of each subgroup is indicated. C) Forest plot displaying the Odd's ratios and 95\% 
1181 confidence intervals $(\mathrm{Cl})$ for the Ki67 response to abemaciclib of the indicated biomarkers. $p$ -

1182

1183

1184

1185

1186

1196

1197

1198

1199

1200

1201

1202

1203

1204

1205 value are also indicated. D) Quantification of p16, pRb, cyclin E1 and cyclin D1 in a cohort of 10 advanced $\mathrm{BC}$ detected by IHC semiquantitatively ( $\mathrm{pRb}$ and $\mathrm{p} 16)$ or by Allred scoring method (cyclin E1 and cyclin D1) displayed according to the patient's response to abemaciclib. Different symbols indicate the treatment. Mean and \pm SEM are indicated. $p$-value, unpaired parametric $t$ test. R: resistant; S: sensitive. E) Forest plot displaying the Odd's ratios and 95\% confidence intervals $(\mathrm{Cl})$ for the patient's response to the study treatments as above. Biomarkers levels were classified as "high" or "low" according to Youden index. High p16 means expression score $\geq 3+$, low pRb means expression score $\leq 1+$ and high cyclin E1/D1 means Allred score $\geq 5$. $p$-values are also indicated.

(1)


Figure 6
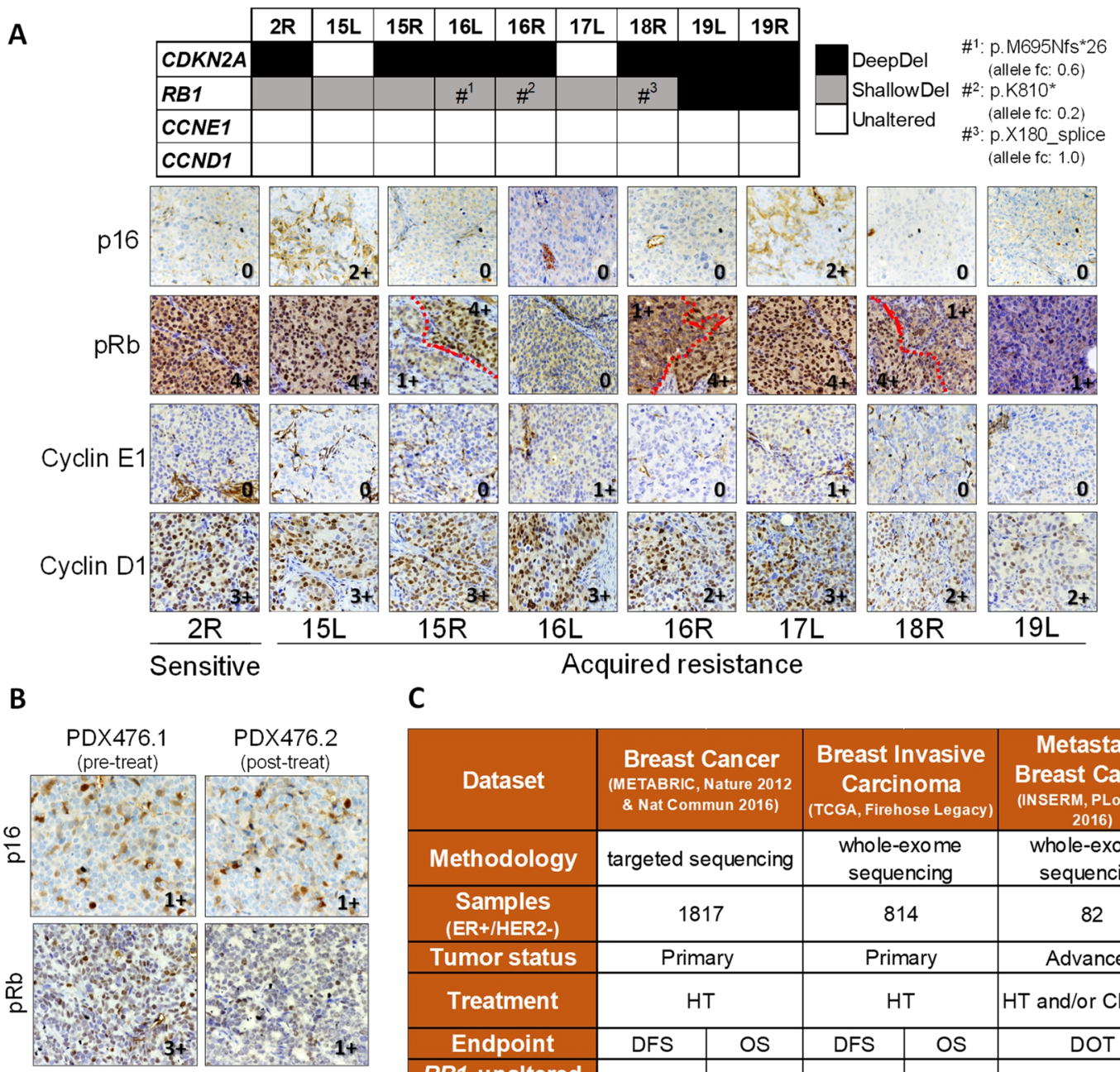

\begin{tabular}{|c|c|c|c|c|c|}
\hline Dataset & \multicolumn{2}{|c|}{$\begin{array}{l}\text { Breast Cancer } \\
\text { (METABRIC, Nature 2012 } \\
\text { \& Nat Commun 2016) }\end{array}$} & \multicolumn{2}{|c|}{$\begin{array}{c}\text { Breast Invasive } \\
\text { Carcinoma } \\
\text { (TCGA, Firehose Legacy) }\end{array}$} & $\begin{array}{c}\text { Metastatic } \\
\text { Breast Cancer } \\
\text { (INSERM, PLos Med } \\
\text { 2016) }\end{array}$ \\
\hline Methodology & \multicolumn{2}{|c|}{ targeted sequencing } & \multicolumn{2}{|c|}{$\begin{array}{c}\text { whole-exome } \\
\text { sequencing }\end{array}$} & $\begin{array}{c}\text { whole-exome } \\
\text { sequencing }\end{array}$ \\
\hline $\begin{array}{c}\text { Samples } \\
\text { (ER+/HER2-) }\end{array}$ & \multicolumn{2}{|c|}{1817} & \multicolumn{2}{|c|}{814} & 82 \\
\hline Tumor status & \multicolumn{2}{|c|}{ Primary } & \multicolumn{2}{|c|}{ Primary } & Advanced \\
\hline Treatment & \multicolumn{2}{|c|}{$\mathrm{HT}$} & \multicolumn{2}{|c|}{$\mathrm{HT}$} & HT and/or CDK4/6i \\
\hline Endpoint & DFS & OS & DFS & OS & DOT \\
\hline $\begin{array}{l}\text { RB1 unaltered } \\
\text { (months) }\end{array}$ & 226 & 169 & 215 & 130 & 118 \\
\hline $\begin{array}{l}\text { RB1 hetloss } \\
\text { (months) }\end{array}$ & 175 & 132 & 139 & 130 & 50 \\
\hline p-value & 0.005 & 0.0007 & 0.7 & 0.1 & 0.008 \\
\hline
\end{tabular}

D

Advanced BC ( $n=582)$

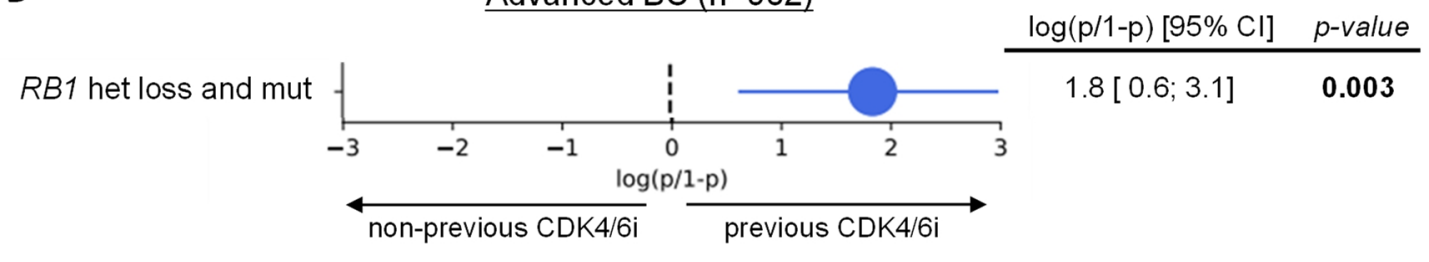

Figure 6. Acquisition of subclonal RB1 mutations in tumors with underlying RB1

1208 heterozygous loss as mechanism of CDK4/6i acquired resistance in BC PDXs. A) On the

1209 top, copy number and mutation status of CDKN2A, RB1, CCNE1 and CCND1 in tumors derived

1210 from PDX244, including an untreated sensitive tumor (2R) or tumors with acquired resistance to

1211 ribociclib (15L to 19R). Deep-Del, CN $<-1$; Shallow-Del, $-1 \leq \mathrm{CN}<-0.4$; Unaltered, $\mathrm{CN} \geq-0.4$.

1212 Hashtags indicate tumors that acquired deleterious mutations in RB1. On the bottom,

1213 representative pictures showing $\mathrm{IHC}$ staining of $\mathrm{p} 16, \mathrm{pRb}$, cyclin $\mathrm{E} 1$ and cyclin $\mathrm{D} 1$ from the 
1214 indicated tumors (bottom). Dashed-red lines mark off areas with different protein staining intensity 1215 and protein scoring is indicated. For 19R there was no FFPE tumor available. Magnification 40x.

1216 B) IHC staining of $\mathrm{p} 16$ and pRb in representative FFPE sections from PDX476.1 and PDX476.2.

1217 Protein scoring is provided. Magnification 40x. C) Clinical outcome expressed in months for the 1218 indicated clinical endpoint in patients with $\mathrm{ER}^{+} / \mathrm{HER} 2^{-}, \mathrm{RB} 1$ unaltered tumors vs. those harboring 1219 tumors with RB1 heterozygous loss. Data was extracted using the cBioportal 1220 (www.cBioportal.org). OS, overall survival; DFS, disease free survival; DOF, days of treatment. D) Association between RB1 double hit alterations (concomitant mutation and deletion) and prior exposure to CDK4/6 inhibitors in metastatic BC patients. The horizontal segment represents the $95 \%$ confidence intervals of the logit value for each test. The size of the circle is proportional to

1224 the negative logarithms of the logit $p$-value.

1225

1226

1227

1228

1229

1230

1231

1232

1233

1234

1235

1236

1237

1238 
Figure 7

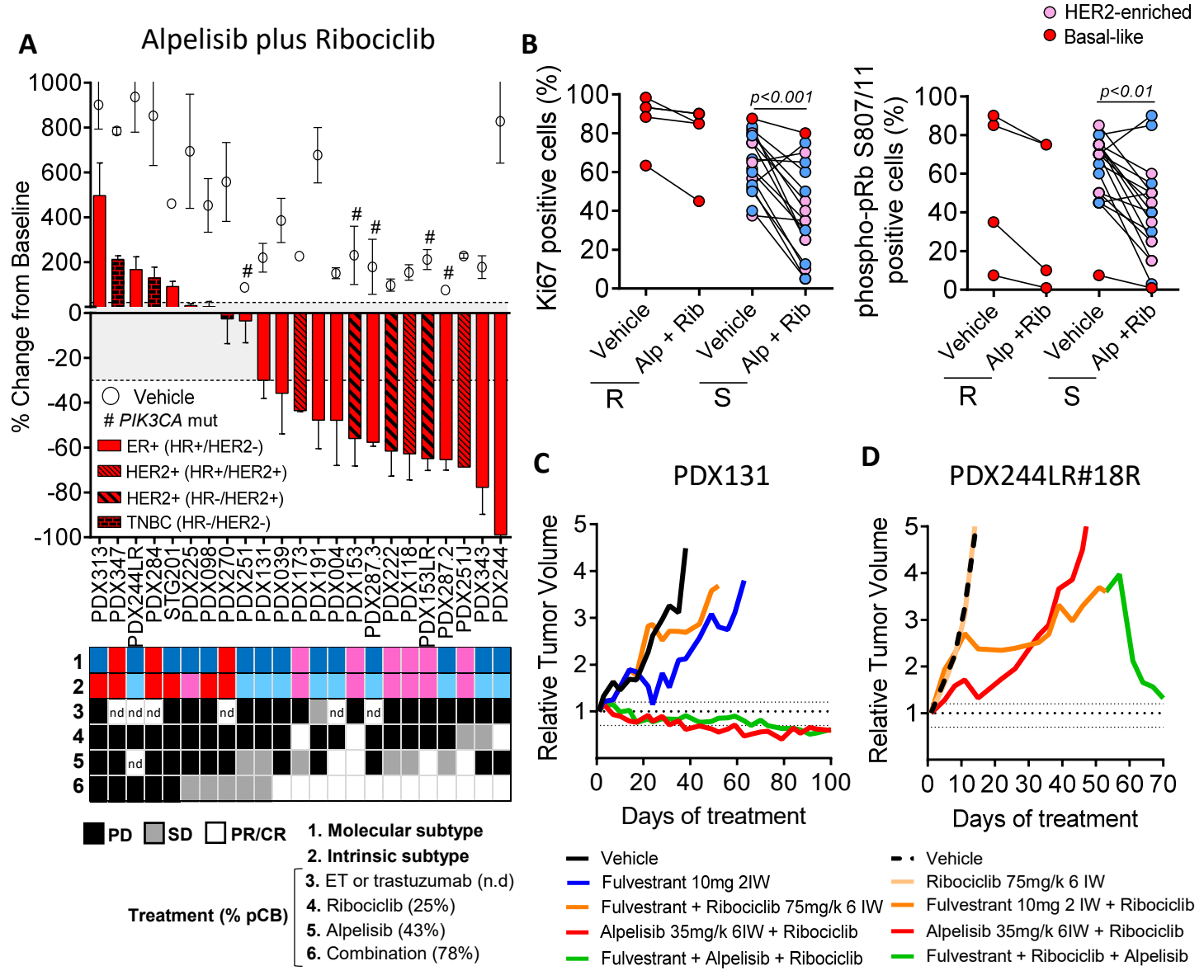

E

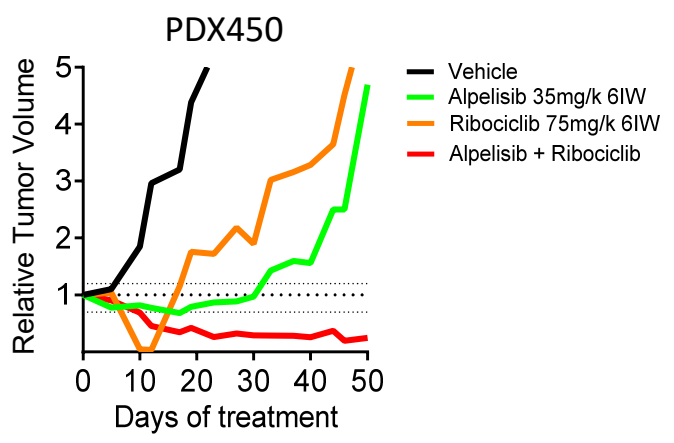

1239

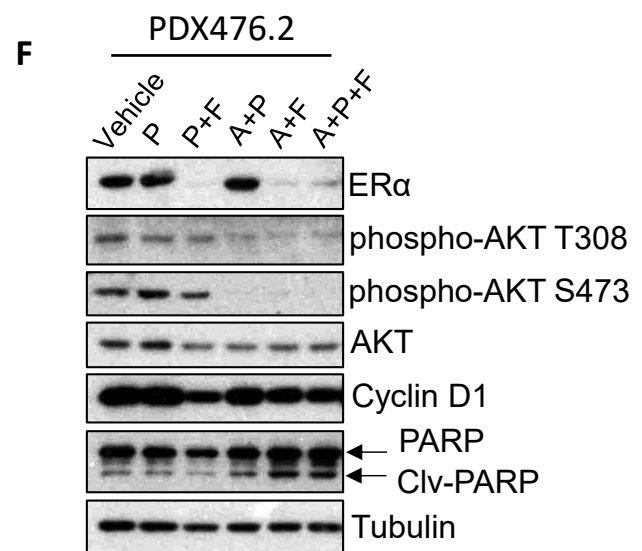

Figure 7. PI3K inhibition sensitizes non-basal like BC PDX to CDK4/6i. A) Waterfall plot showing the growth of 23 PDX treated with ribociclib $75 \mathrm{mg} / \mathrm{kg}$ plus alpelisib $35 \mathrm{mg} / \mathrm{kg}$ (bars) and vehicle (circles). The percentage change from the initial volume is shown at day 35 of treatment. Dashed lines indicate the range of PD $(>20 \%)$, SD $(20 \%$ to $-30 \%)$ and PR/CR $(<-30 \%)$. The molecular subtypes are indicated. Hashtags indicate models harboring mutations in PIK3CA. Data represent mean and error bars \pm SEM. Boxes underneath show the molecular and intrinsic tumor's subtypes as well as their responses to the indicated treatments. The preclinical benefit 
1247 to each drug is indicated as percentage in brackets. n.d, not determined. B) IHC analysis of Ki67

1248 (left graph) and phospho-pRb S807/811 (right graph) in vehicle and alpelisib plus ribociclib-

1249 treated PDXs in relationship to the PDXs response to alpelisib plus ribociclib. For illustration

1250 purposes, the mean value of each PDX was plotted; however, for the statistical analysis all

1251 technical replicates were used. $p$-values are based on Mann-Whitney $U$ test. Different colors

1252 represent the tumor's intrinsic subtype. R, resistant; S, sensitive; Alp, alpelisib; Rib, ribociclib. C),

1253 D) and E) Relative tumor growth of the ribociclib-resistant PDX131, PDX244LR\#18R and PDX450

1254 after treatment with the indicated drug(s) for the indicated period of time. Dashed lines indicate

1255 the range of PD (>1.2), SD (1.2 to -0.7$)$ and PR/CR (<-0.7). F) Immunoblot of indicated the

1256 proteins in PDC476.2 treated with vehicle or $500 \mathrm{nM}$ palbociclib as single-agent or combined with

$1257100 \mathrm{nM}$ fulvestrant and/or $2.5 \mu \mathrm{M}$ alpelisib in ex vivo cultures for 48 hours. 
A

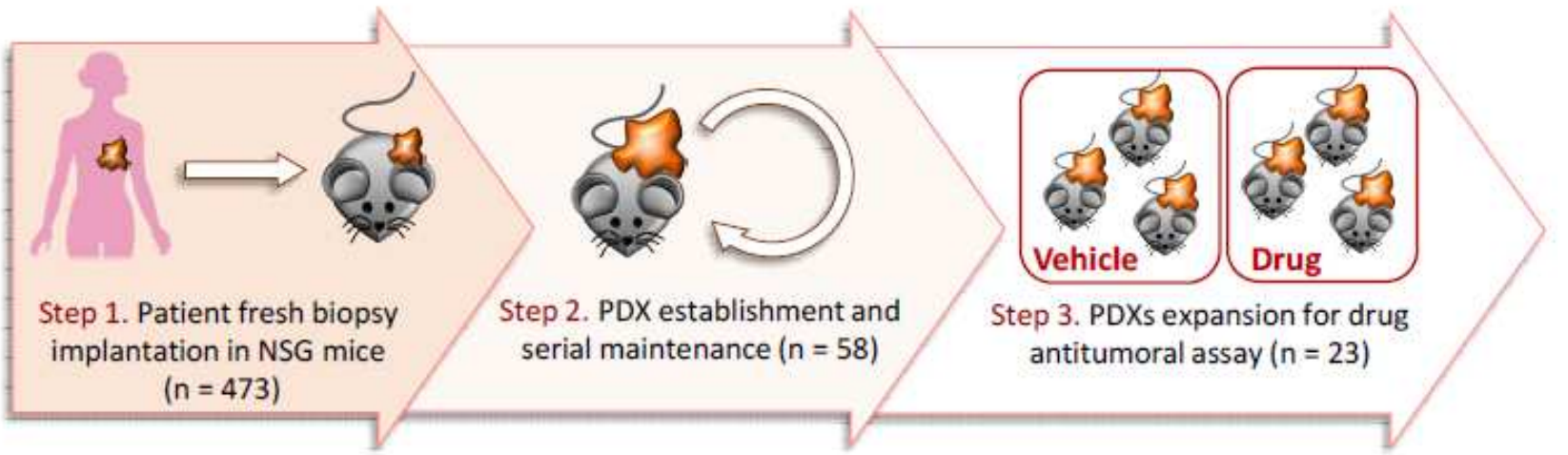

B

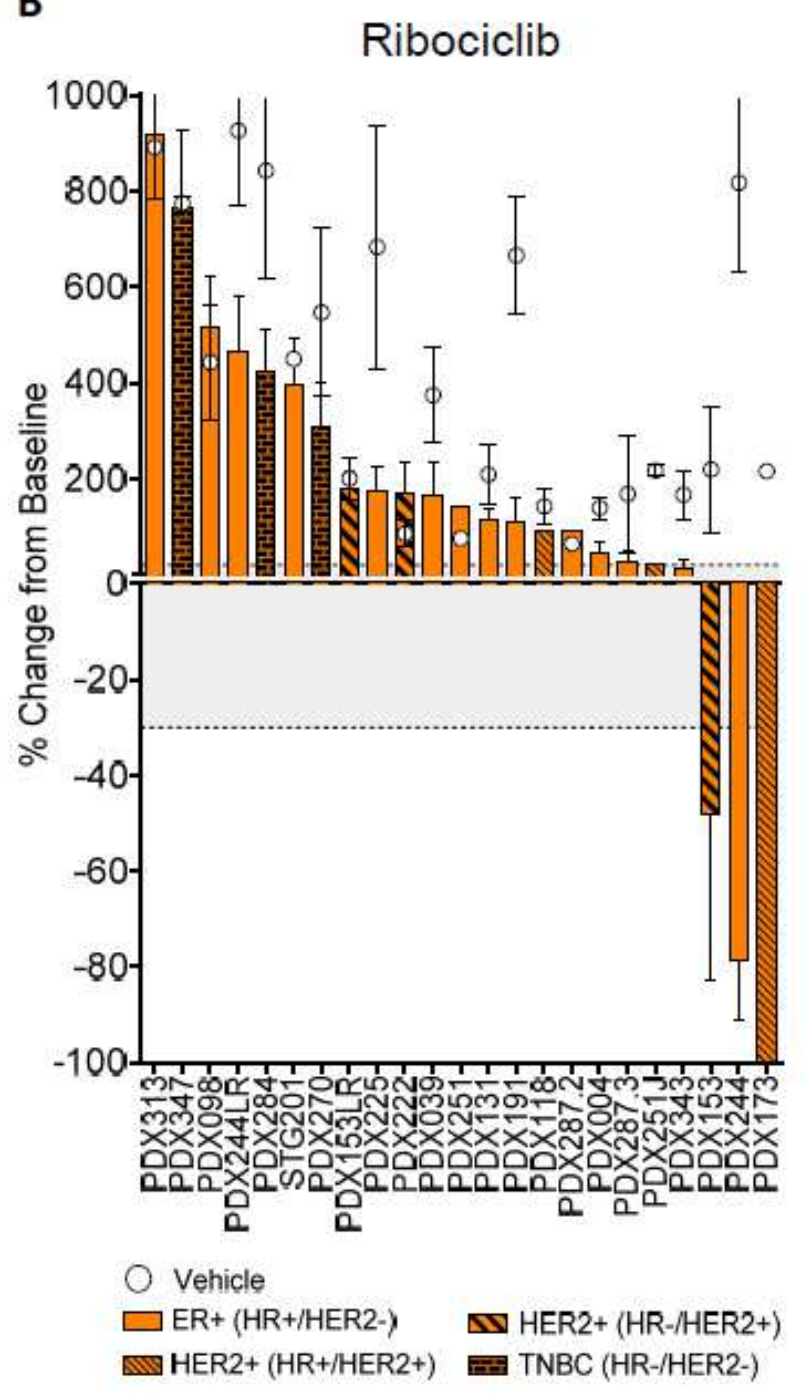

C

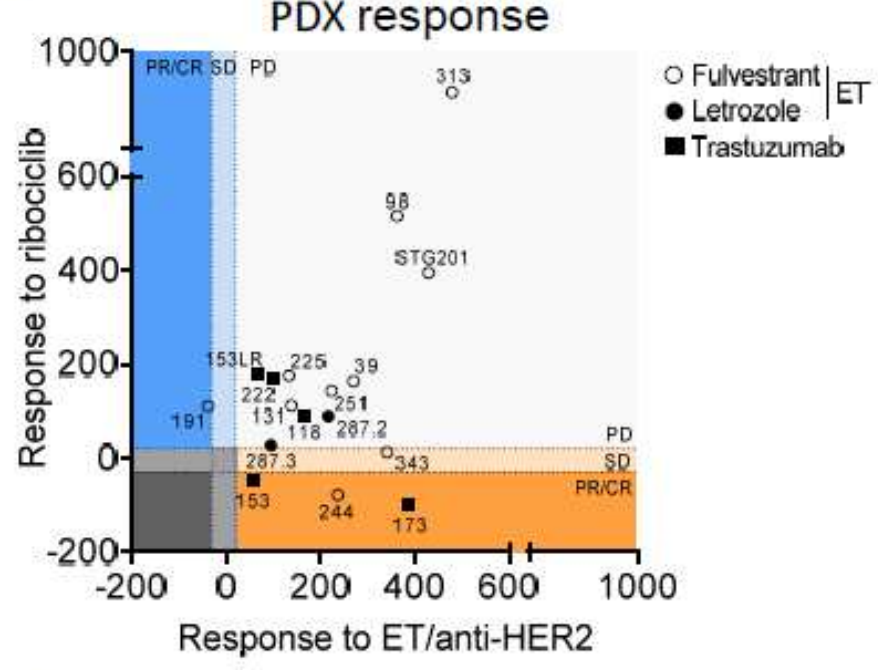

D

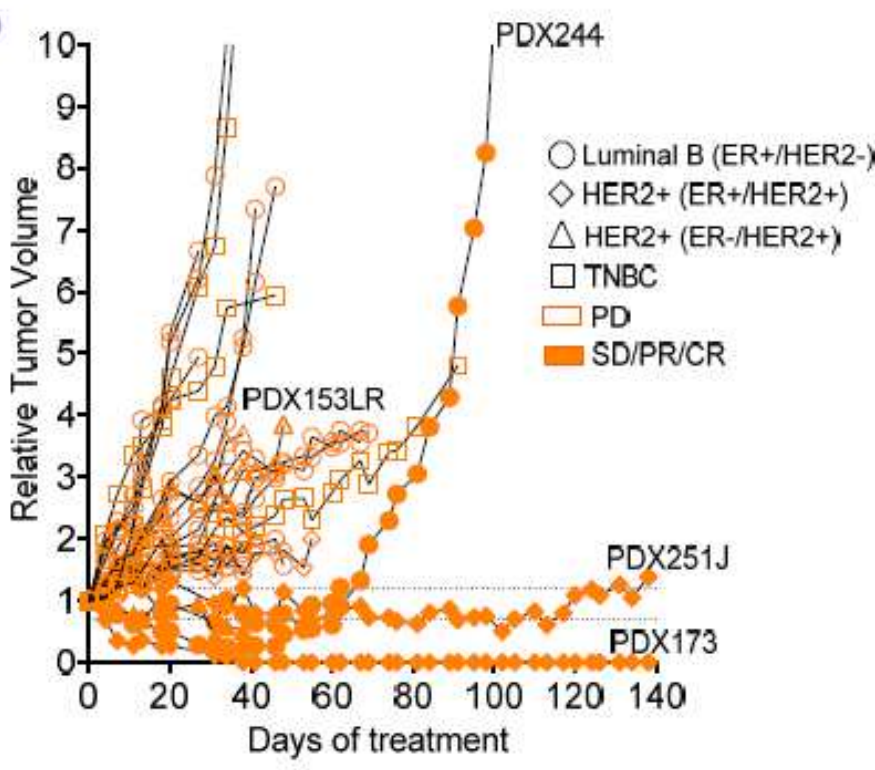

\section{Figure 1}

Ribociclib monotherapy has higher antitumor activity than other targeted agents in ER+ and HER2+ BC PDXs. A) Workflow depicting the generation of BC PDX models from BC patient samples and its subsequent expansion for targeted treatment screening. B) Waterfall plot representing the growth of 23 
PDX treated with ribociclib $75 \mathrm{mg} / \mathrm{kg}$ (bars) and vehicle (circles). The percentage change from the initial volume is shown at day 35 of treatment. Dashed lines indicate the range of PD (>20\%), SD (20\% to $-30 \%)$ and PR/CR (<-30\%). The molecular subtypes are indicated. Data represent means and error bars \pm SEM. C) Antitumor response of ribociclib (y-axis) vs. other targeted agents (x-axis; endocrine therapy or trastuzumab) in PDXs represented as the percentage of tumor volume change compared to the initial tumor volume. Symbol shapes represent the different targeted therapies. D) Spaghetti plot showing the relative tumor volume change along time in 23 BC PDX treated with ribociclib $75 \mathrm{mg} / \mathrm{kg}$. Ribociclibsensitive models are represented with fill symbols and ribociclib-resistant with empty symbols. Symbol shapes represent the PDX's molecular subtypes. Dashed lines indicate the range of PD (>1.2), SD (1.2 to $-0.7)$ and PR/CR (<-0.7). Acquisition of ribociclib resistance in PDX244 (PDX244LR) is shown. 


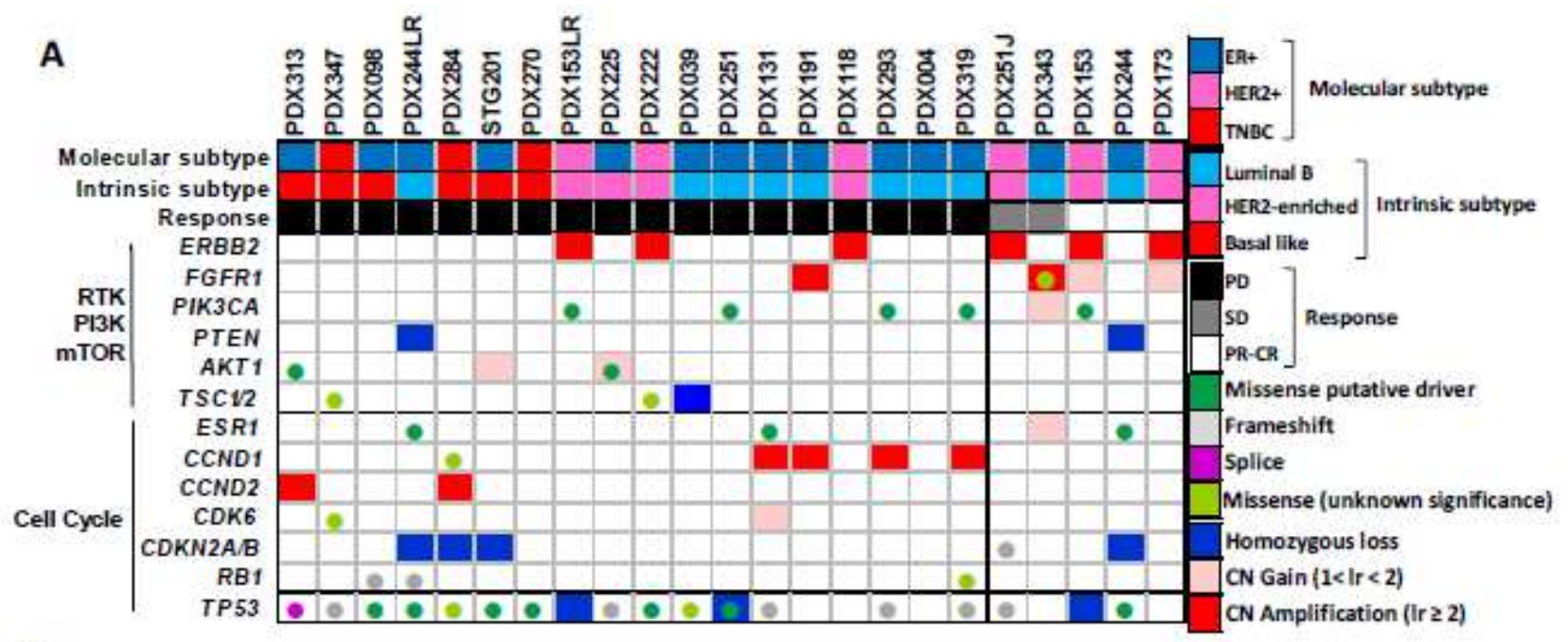

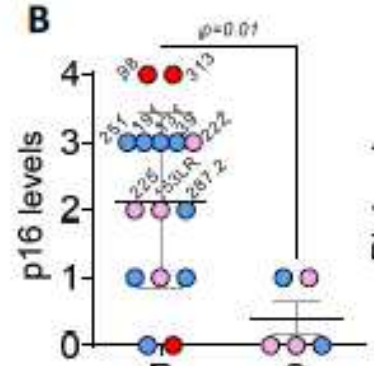

$\mathrm{R}$
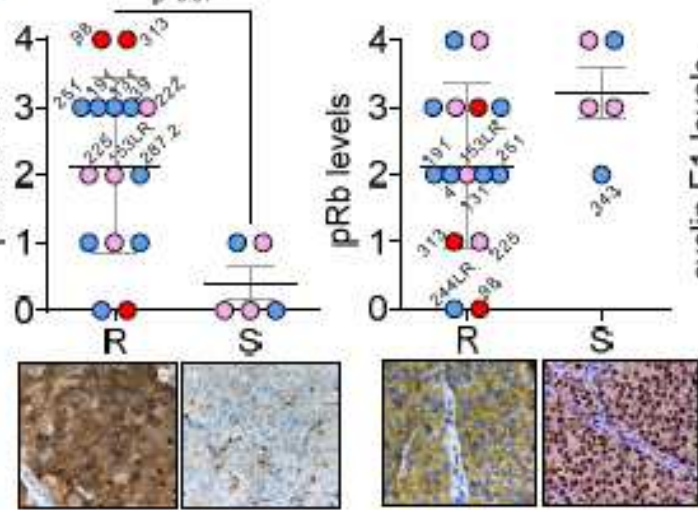

C

p16 high

$\mathrm{pRb}$ low

cyclin $\mathrm{E} 1$ high

cyclin D1 high

Sensitivity (\%)

Specificity (\%)

PPV (\%)

NPV (\%)

Accuracy (\%)

\begin{tabular}{|c|c|c|c|c|}
\hline & Pro & ein & $\overline{m R}$ & $\overline{N A}$ \\
\hline $\begin{array}{c}\text { ER+ Breast Invasive Carcinoma } \\
(\text { TCGA) } n=814\end{array}$ & $\log 2$ OR & p-value & $\log 2$ OR & p-value \\
\hline p16 high and pRb low & 2.8 & 0.006 & $>3$ & $<0.001$ \\
\hline pRb low and Cyclin E1 high & 2.9 & 0.02 & $>3$ & $<0.001$ \\
\hline p16 high and Cyclin E1 high & $<-3$ & 0.8 & $>3$ & $<0.001$ \\
\hline
\end{tabular}

High: prot/mRNA > 1.5 SD above the mean; Low: prot/mRNA < - 1.5 SD under the mean
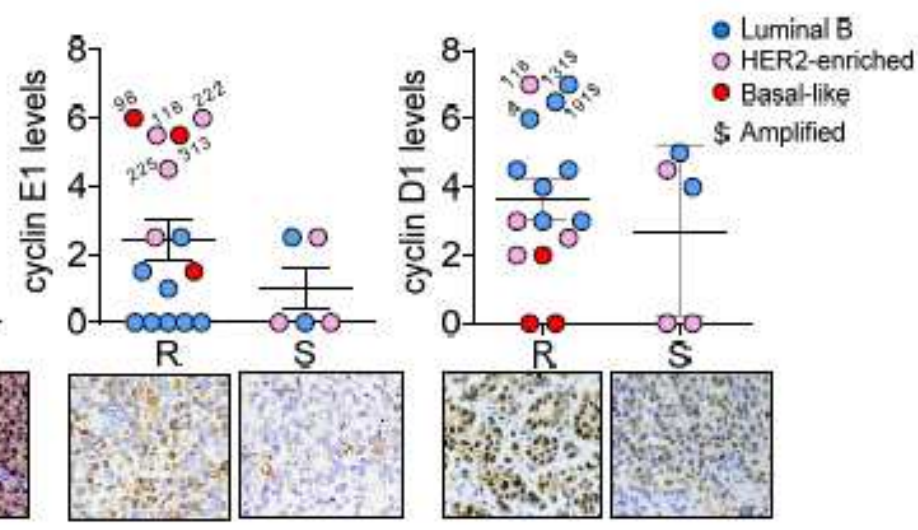

E

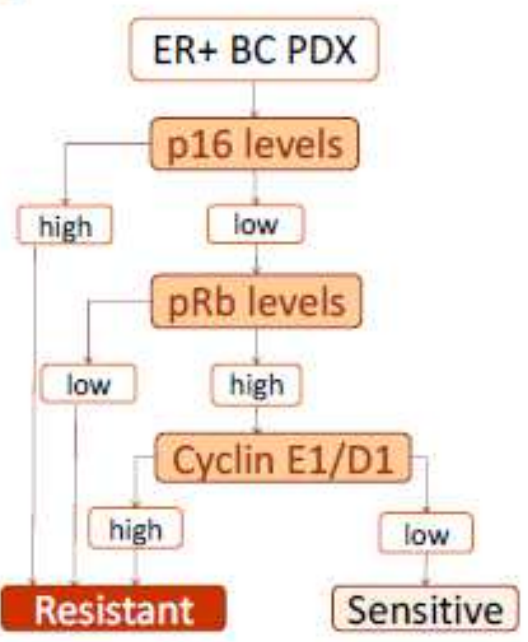

\section{Figure 2}

PDXs expressing high p16 are resistant to ribociclib. A) Summary of genetic alterations in the PDX panel from Figure 1B, including the PDX subtype classification, based on IHC (Molecular subtype) or PAM50 analysis (intrinsic subtype), and the response to CDK4/6 inhibitors. Genes with similar function such as TSC1/TSC2 or CDKN2A/CDKN2B were considered as one single feature. B) Quantification of IHC staining for p16, pRb, cyclin E1 and cyclin D1 in 23-untreated PDX in relationship with ribociclib-response. 
Semiquantitative analysis was performed for $\mathrm{pRb}$ and $\mathrm{p} 16$, or the Allred scoring method for cyclin $\mathrm{E} 1$ and cyclin D1 in relationship with ribociclib-response. Different colors indicate the PDX intrinsic subtype and \$ indicates the models harboring gene amplification. Mean and \pm SEM are indicated. $p$-value, unpaired parametric t-test. The pictures underneath are representative bright field images of high/low staining of each protein. Magnification 40x. R: resistant; S: sensitive. C) Prediction analysis of the indicated biomarker(s) to classify a PDX as resistant or sensitive to ribociclib based on their expression levels and according to Youden index. High p16 means expression score $\geq 2+$, low pRb means expression score $\leq 1+$ and high cyclin E1/D1 means Allred score > 4/6. D) Co-occurrence of altered p16, pRb and cyclin E1 expression levels in a cohort of $814 \mathrm{ER}+$ breast invasive carcinomas from the TCGA database using cBioportal (www.cBioportal.org). The cut-off for high versus low protein/mRNA levels is indicated. OR: odd's ratio; prot: protein; SD: standard deviation. E) Consort flow diagram for classifying the PDX responses to ribociclib based on the molecular subtype, p16, pRb and cyclin E1/D1 scores. 
A

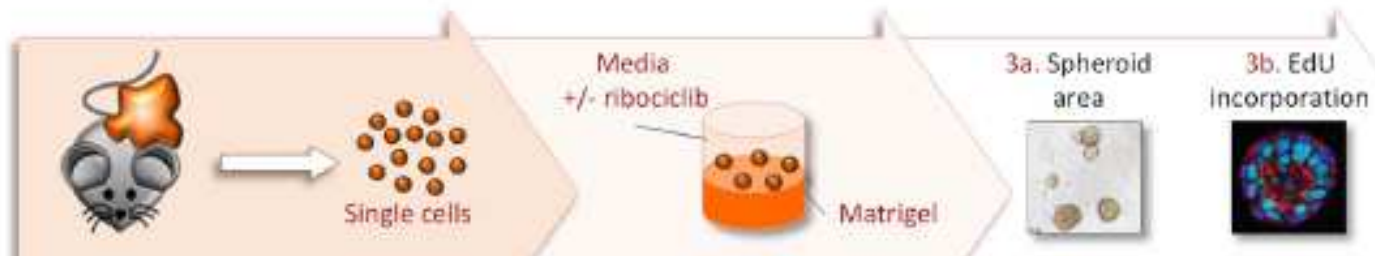

Step 1. Mechanical and enzymatic

Step 2. Cells seeded on top disaggregation Matrigel

Step 3. Analysis of ribociclib antiproliferative activity

B

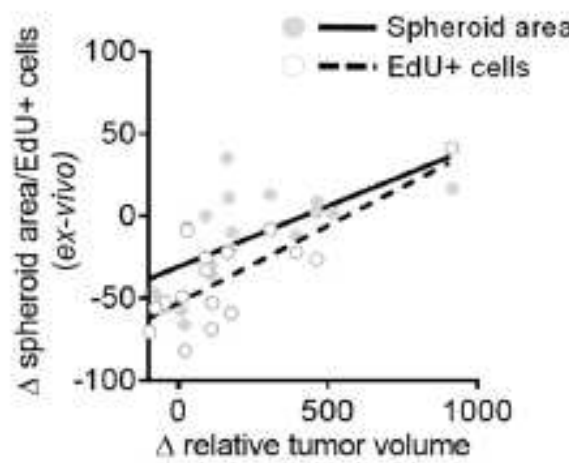

(in vivo)

\begin{tabular}{|r|c|c|}
\cline { 2 - 3 } \multicolumn{1}{c|}{} & $\Delta$ Spheroid area & $\Delta$ EdU+ cells \\
\hline r & 0.8 & 0.6 \\
\hline p-value & 0.0001 & 0.02 \\
\hline $95 \%$ ic & $0.5-0.9$ & $0.1-0.8$ \\
\hline
\end{tabular}

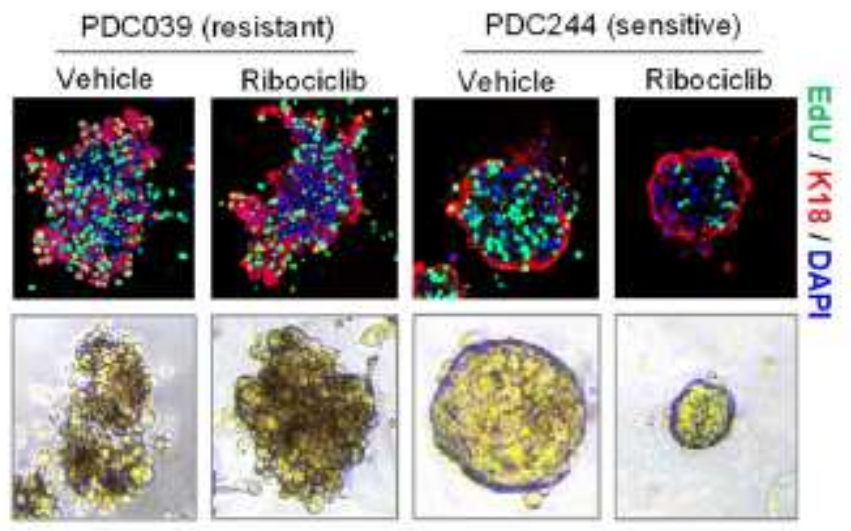

C

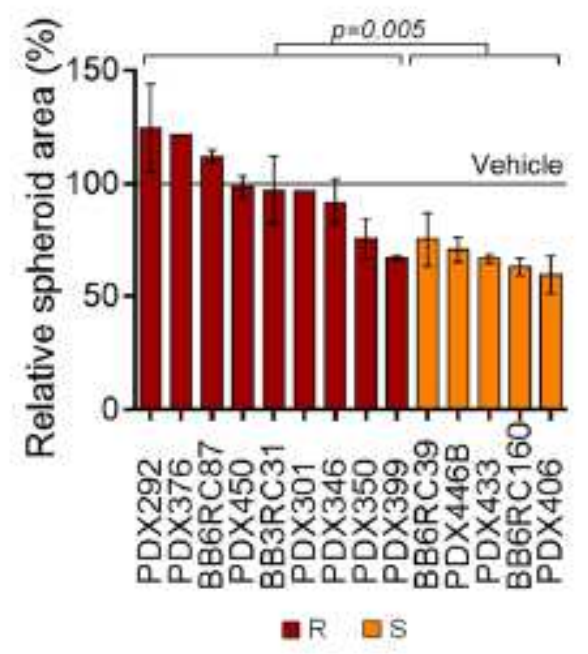

D

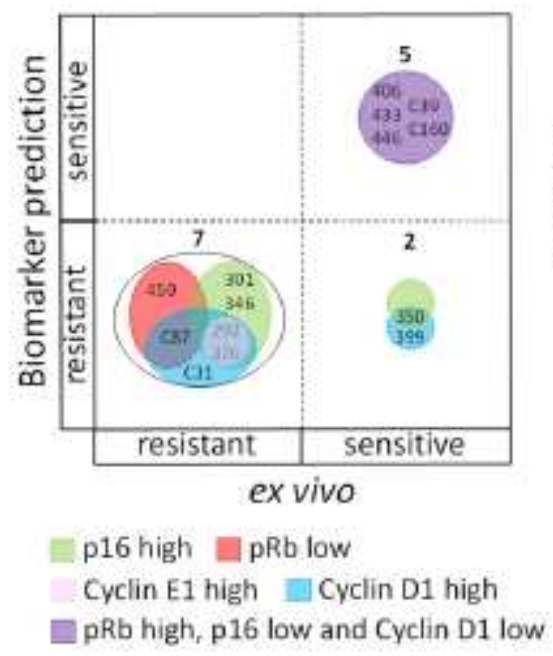

E

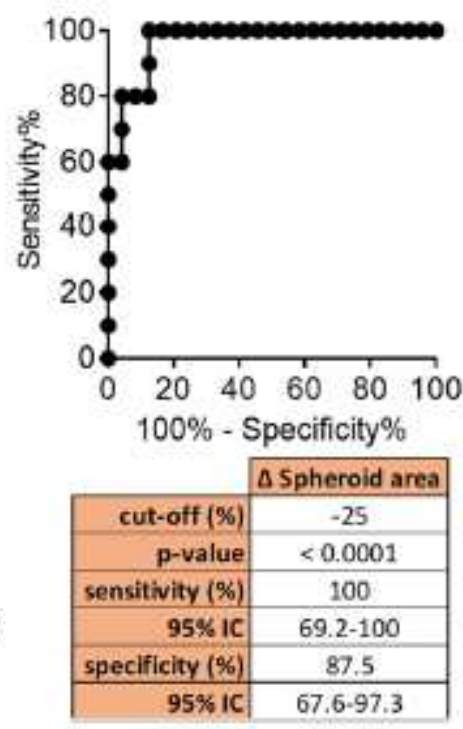

\section{Figure 3}

Biomarker validation in short-term patient-derived tumor cells (PDCs). A) Workflow depicting the generation of BC PDCs short-term ex vivo cultures from PDXs and the subsequent analysis of ribociclib response using two different read-outs. B) Correlation analysis of the ex vivo response of PDCs (y-axis) vs. the in vivo response of the corresponding PDXs ( $x$ axis), measured as spheroid area change (empty dots) or the change in EdU incorporation (filled dots) after ribociclib treatment vs. the change in the relative tumor volume. The Spearman's coefficient $(r)$, $p$-value and $95 \%$ of confidence interval $(95 \% \mathrm{Cl})$ for 
each read-out are summarized below the graph. Representative images of one ribociclib-resistant (PDC039) and one ribociclib sensitive (PDC244) model treated with vehicle or ribociclib are shown on the right panel, namely EdU/K18 staining by confocal microscopy and bright field. EdU is shown in green, cytokeratin 18 (K18) in red and DAPI in blue. Magnification 40x. C) Relative spheroid area in 14 PDC models classified as resistant (maroon) or sensitive (orange) according to the composite biomarker. Treatment with $1 \mu \mathrm{M}$ ribociclib for 7 days. Relative data to the vehicle control $(100 \%)$ is represented as mean of three independent experiments \pm SEM. $p$-value, unpaired parametric $t$ test. D) Concordance analysis of PDXs' responses to ribociclib based on biomarker prediction ( $y$ axis) vs. the ex vivo response ( $\mathrm{x}$-axis). Biomarkers are represented by circles with different colors and the number of PDX within each category is indicated. E) ROC-curve of the spheroid area increment for ribociclib response prediction in 37 BC PDCs. The p-values and the $95 \%$ confidence interval $(95 \% \mathrm{Cl})$ are summarized below the graph. 
A

\begin{tabular}{|c|c|c|c|c|}
\hline \multicolumn{2}{|c|}{$\begin{array}{c}\text { Treatment } \\
\text { Cell lines }\end{array}$} & $\begin{array}{c}\text { Fulvestrant } \\
n M(\mathrm{fc})\end{array}$ & $\begin{array}{c}\text { Ribociclib } \\
\mathrm{HM} \text { (fc) }\end{array}$ & $\begin{array}{c}\text { Combination } \\
\mathrm{nM} \mu \mathrm{\mu M} \text { (fic) }\end{array}$ \\
\hline \multirow{2}{*}{ T470 } & MOCK & 0.2 & 2.2 & 0.3 \\
\cline { 2 - 5 } & $\mathrm{P} 16$ & $0.9(4.5)$ & $44.9(20.4)$ & $1.8(6)$ \\
\hline
\end{tabular}
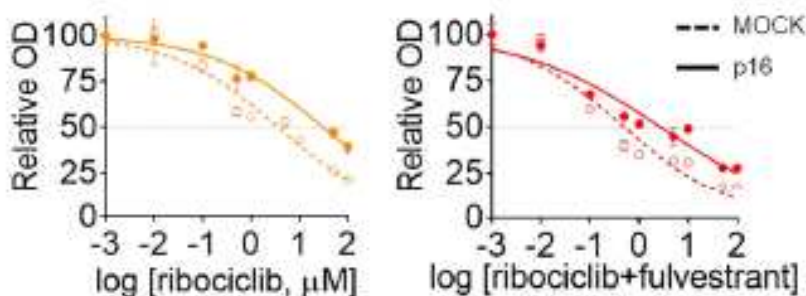

$(\mu \mathrm{M} ; \mathrm{nM})$
B

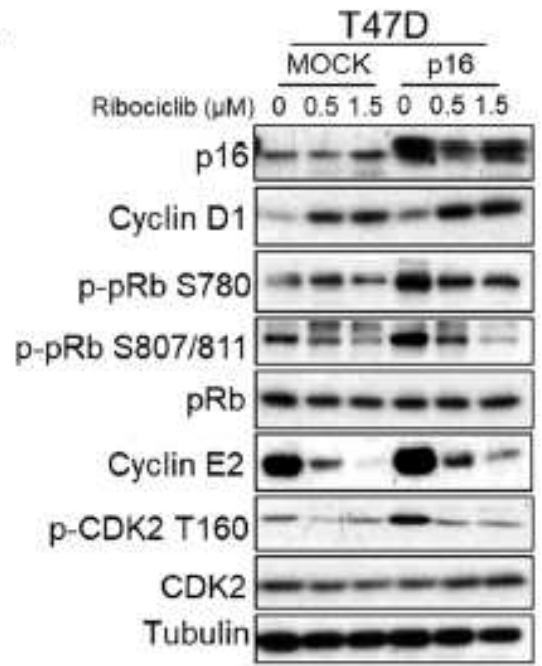

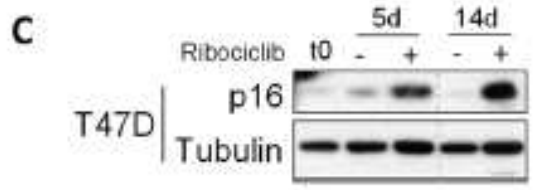

E

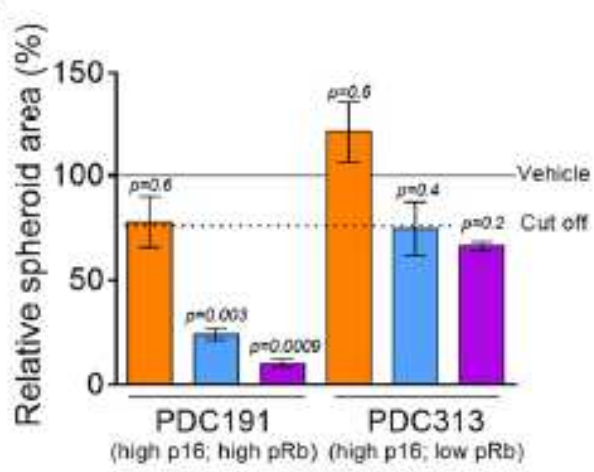

$\square$ Ribociclib $\square$ P18N003 $\square$ P18N003 + Ribociclib

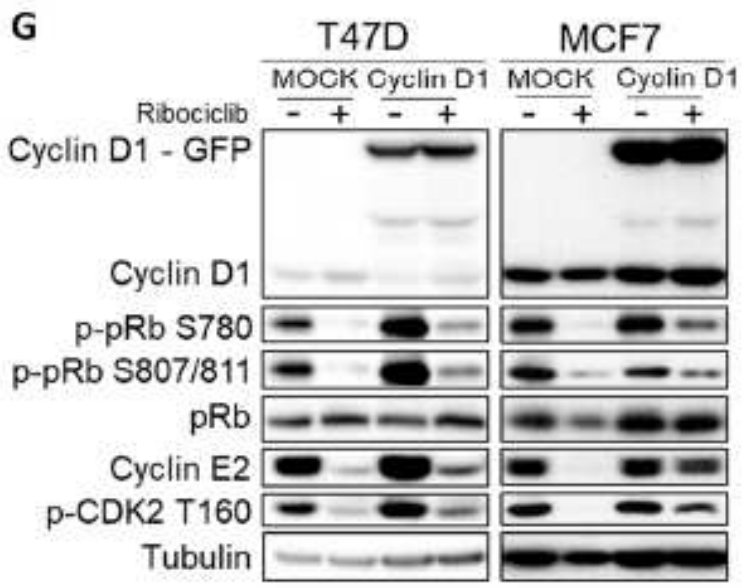

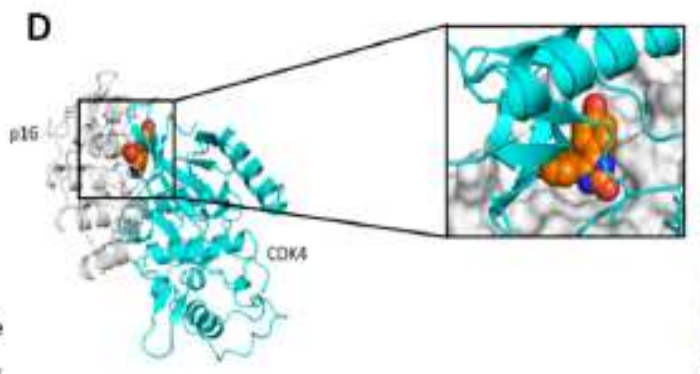

$\mathbf{F}$

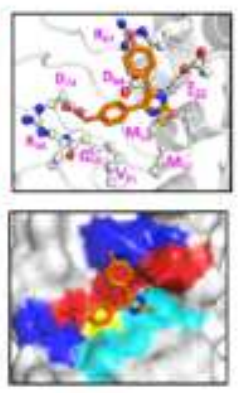

\begin{tabular}{|c|c|c|c|c|}
\hline Cell lin & Treatment & $\begin{array}{c}\text { Fulvestrant } \\
\text { nM (fc) }\end{array}$ & \begin{tabular}{|c|} 
Ribociclib \\
$\mu \mathrm{M}(\mathrm{fc})$
\end{tabular} & $\begin{array}{l}\text { Combination } \\
\mathrm{nM} / \mu \mathrm{M} \text { (fc) }\end{array}$ \\
\hline \multirow{2}{*}{$\mathrm{T} 47 \mathrm{D}$} & MOCK & 0.5 & 4.4 & 0.1 \\
\hline & cyclin D1 & $2(4.0)$ & $13.7(3.1)$ & $0.5(5.0)$ \\
\hline
\end{tabular}

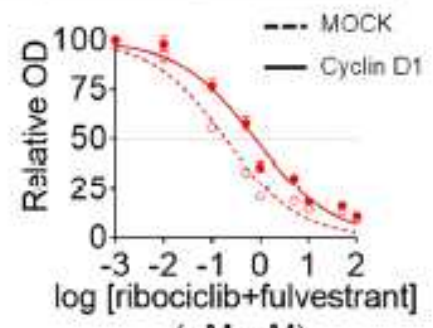

$(\mu \mathrm{M} ; \mathrm{nM})$
H

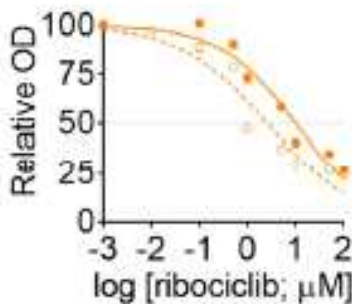

\section{Figure 4}

p16 and cyclin D1 overexpression attenuate the response to ribociclib in ER+ BC cell lines. A) Halfmaximal inhibitory concentration (IC50) values of ribociclib, fulvestrant and the combination of T47D cells overexpressing p16 (CDKN2A) and fold-change values ( $\mathrm{fc}$; in brackets) compared to controls (MOCK), evaluated after 6-days dose-response experiments as shown underneath. At least three independent experiments were conducted with three technical replicates per condition. B) Immunoblot of 
the indicated proteins in MOCK and p16 overexpressing T47D cells untreated or treated with ribociclib for 5 days at the indicated concentrations. C) Immunoblot of the indicated proteins in an enrichment assay. D) Comparison of structural models built in this study for the complexes of p16 bound to P18IN003 and p16 bound to CDK4, showing that CDK4 and P18IN003 share the same binding pocket on p16 and hence CDK4 cannot bind to p16 when P18IN003 is bound to p16 (grey cartoon is p16, orange spheres represent $\mathrm{P} 18 \mathrm{IN} 003$, cyan cartoon is CDK4); upper zoomed view is the binding pocket with p16 shown as grey surface and lower zoomed views are the same, highlighting the residues in the binding pocket of p16 as sticks and the hydrogen bonds made between P18IN003 and p16 shown as black dashed lines. E) Relative spheroid area of PDC191 and PDC313 after treatment with $1 \mu \mathrm{M}$ ribociclib, 20nM P18IN003 and the combination in ex vivo cultures for 7 days. Data are presented as means of three independent experiments \pm SEM. p-values are based on the one way ANOVA test with Tukey's method correction compared with the vehicle (black line). Dashed line indicates the optimal cut-off established in Figure 3E. p16 and pRb scores of each PDC are indicated. F) Half-maximal inhibitory concentration (IC50) values of ribociclib, fulvestrant and the combination of T47D cells overexpressing cyclin D1 (CCND1) and foldchange values (fc; in brackets) compared to controls (MOCK), evaluated after 6-days dose-response experiments as shown underneath. At least three independent experiments were conducted with three technical replicates. G) Immunoblot of indicated proteins in control (MOCK) and cyclin D1 (CCND1) overexpressing T47D (left panel) and MCF7 (right panel) cells untreated or treated with $0.5 \mu \mathrm{M}$ ribociclib for 48 hours. H) Immunoblot of the indicated proteins in an enrichment experiment. 
A

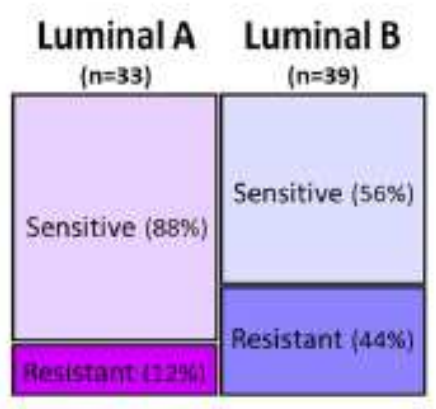

C

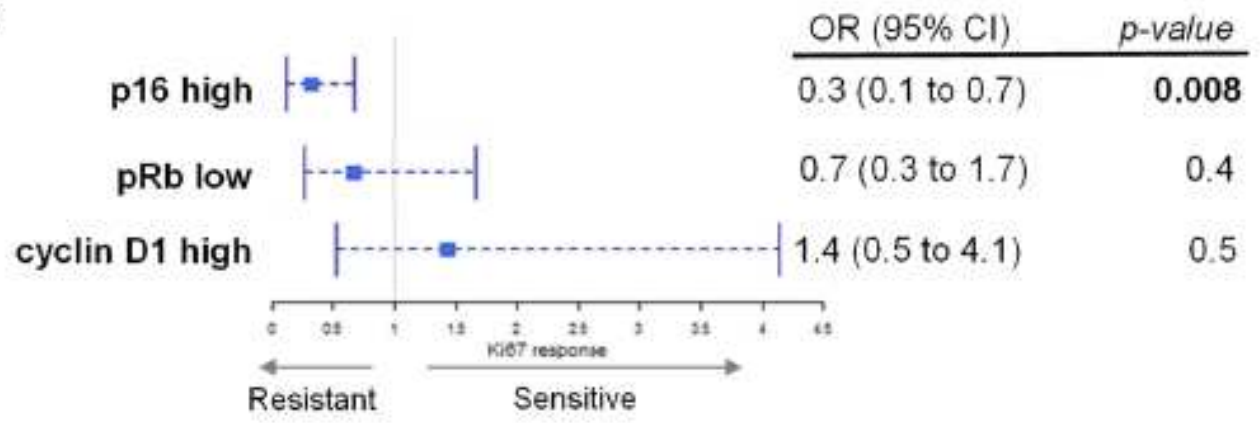

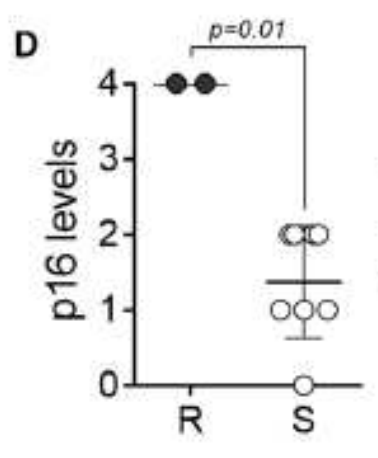

E
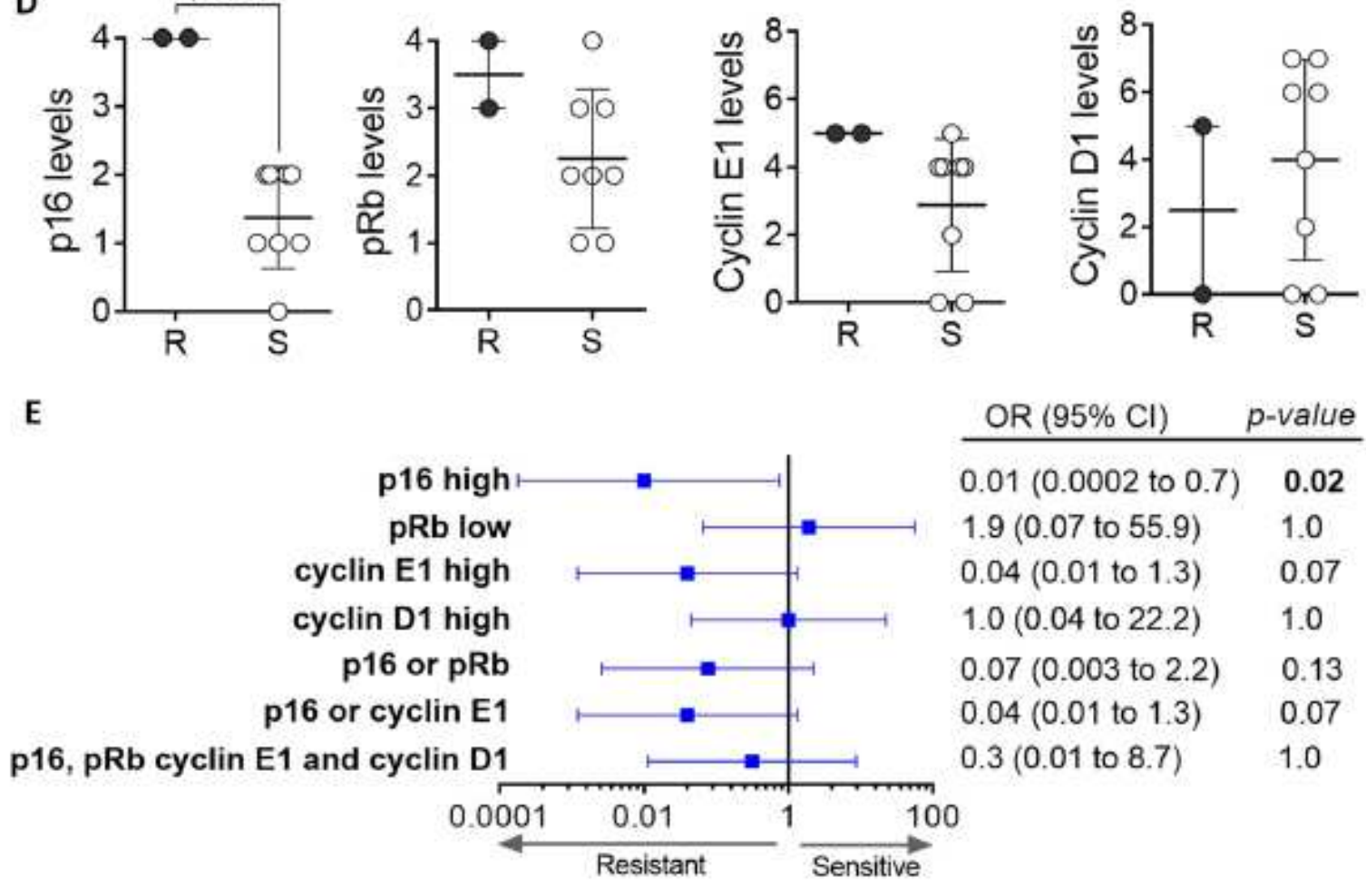

\section{Figure 5}

High p16 levels associated with lack of response to CDK4/6i in ER+ BC patients. A) Representation of the percentage distribution of Luminal A/B tumors vs. sensitivity to abemaciclib after 15 days of treatment in the neoadjuvant setting in the ABC-POP trial. Tumors were classified as Luminal A if \%Ki67<15 or as Luminal B if \% Ki67 $\geq 15$. Tumors showing In Ki67 < 1 at day 15 were considered sensitive and those with In Ki67 $\geq 1$ were resistant to the studied drug. B) Logistic model to evaluate the effect of p16 on the 
response to abemaciclib. Mean value of each subgroup is indicated. C) Forest plot displaying the Odd's ratios and 95\% confidence intervals $(\mathrm{Cl})$ for the Ki67 response to abemaciclib of the indicated biomarkers. $p$ value are also indicated. D) Quantification of p16, pRb, cyclin E1 and cyclin D1 in a cohort of 10 advanced $\mathrm{BC}$ detected by IHC semiquantitatively ( $\mathrm{pRb}$ and $\mathrm{p} 16)$ or by Allred scoring method (cyclin E1 and cyclin D1) displayed according to the patient's response to abemaciclib. Different symbols indicate the treatment. Mean and \pm SEM are indicated. $p$-value, unpaired parametric $t$ test. R: resistant; S: sensitive. E) Forest plot displaying the Odd's ratios and 95\% confidence intervals (Cl) for the patient's response to the study treatments as above. Biomarkers levels were classified as "high" or "low" according to Youden index. High p16 means expression score $\geq 3+$, low pRb means expression score $\leq 1+$ and high cyclin E1/D1 means Allred score $\geq 5$. p-values are also indicated. 
A

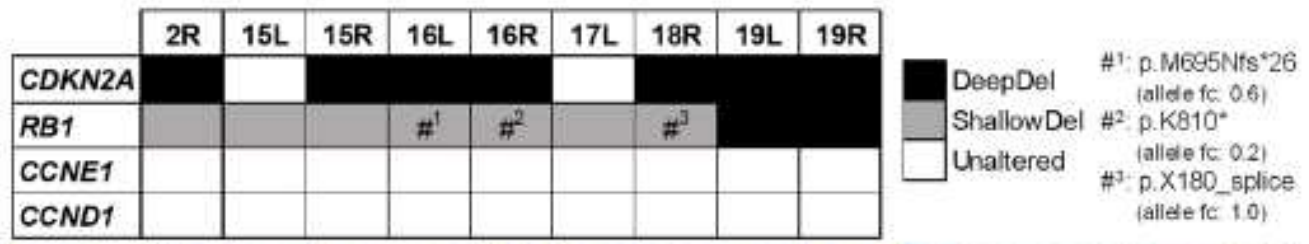

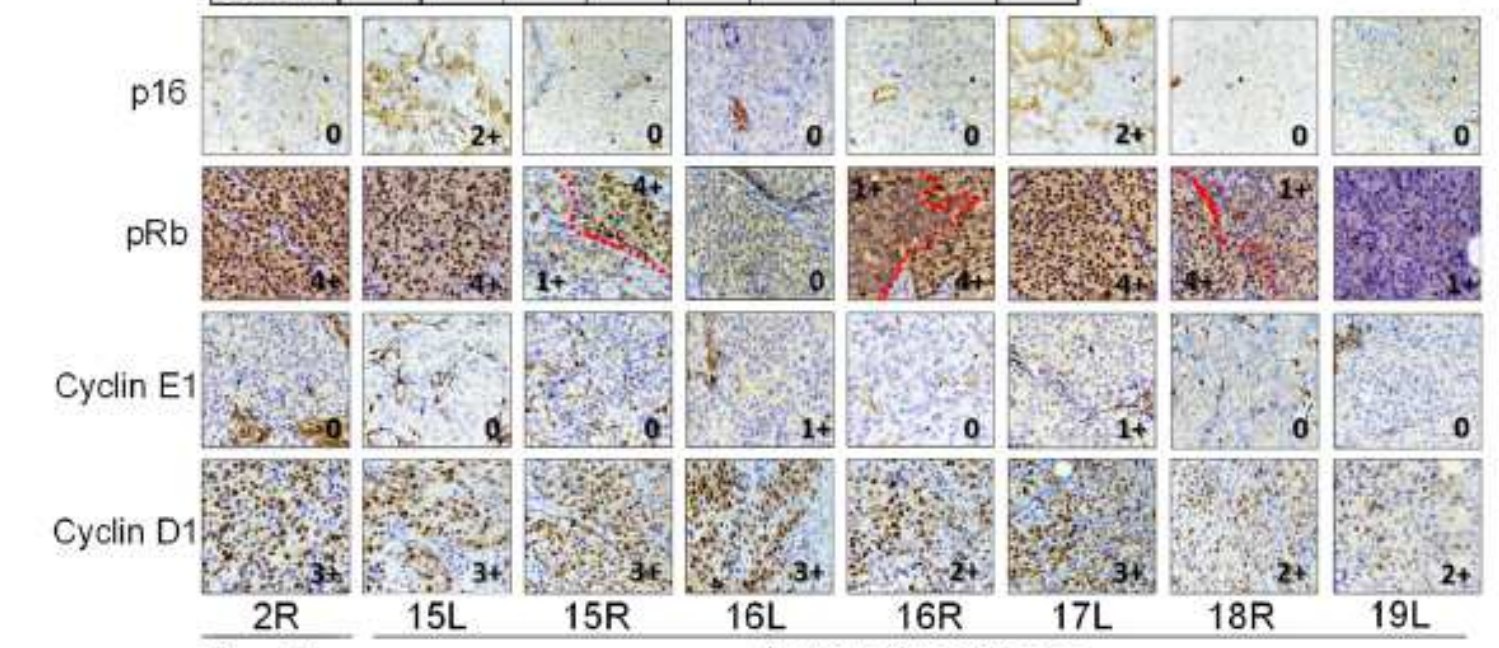

B

\section{Sensitive}

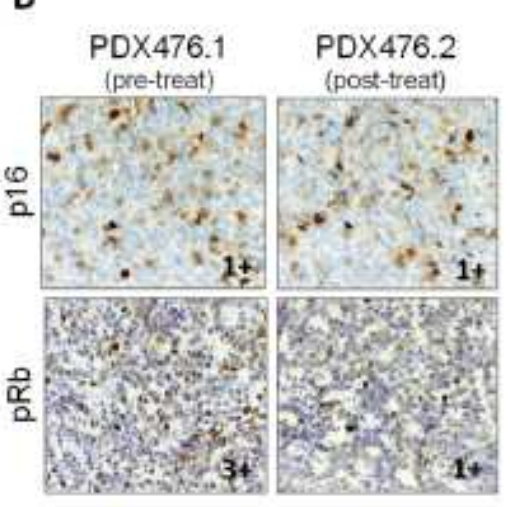

C

Acquired resistance

\begin{tabular}{|c|c|c|c|c|c|}
\hline Dataset & \multicolumn{2}{|c|}{$\begin{array}{l}\text { Breast Cancer } \\
\text { METABRAc, Naturn } 2012 \\
8 \text { Nht Commun 2016) }\end{array}$} & \multicolumn{2}{|c|}{$\begin{array}{c}\text { Breast Invasive } \\
\text { Carcinoma } \\
\text { (TCGA. Firnose Legacy) }\end{array}$} & $\begin{array}{c}\text { Metastatic } \\
\text { Breast Cancer } \\
\text { (INSERAM, PLo5 Mnd } \\
\text { 2016) }\end{array}$ \\
\hline Methodology & \multicolumn{2}{|c|}{ targeted sequencing } & \multicolumn{2}{|c|}{$\begin{array}{l}\text { Whole-exome } \\
\text { sequencing }\end{array}$} & $\begin{array}{l}\text { whole-exome } \\
\text { sequencing }\end{array}$ \\
\hline $\begin{array}{l}\text { Samples } \\
\text { (ER+/HER2-) }\end{array}$ & \multicolumn{2}{|c|}{1817} & \multicolumn{2}{|c|}{814} & 82 \\
\hline Tumor status & \multicolumn{2}{|c|}{ Primary } & \multicolumn{2}{|c|}{ Primary } & Advanced \\
\hline Treatment & \multicolumn{2}{|c|}{ HT } & \multicolumn{2}{|c|}{ HT } & $\mathrm{HT}$ and/or CDK4/6i \\
\hline Endpoint & DFS & os & DFS & os & DOT \\
\hline $\begin{array}{l}\text { RB1 unaltered } \\
\text { (monthy) }\end{array}$ & 226 & 169 & 215 & 130 & 118 \\
\hline $\begin{array}{l}\text { RBY hetloss } \\
\text { (months) }\end{array}$ & 175 & 132 & 139 & 130 & 50 \\
\hline p-value & 0.005 & 0.0007 & 0.7 & 0.1 & 0.008 \\
\hline
\end{tabular}

Advanced $B C(n=582)$

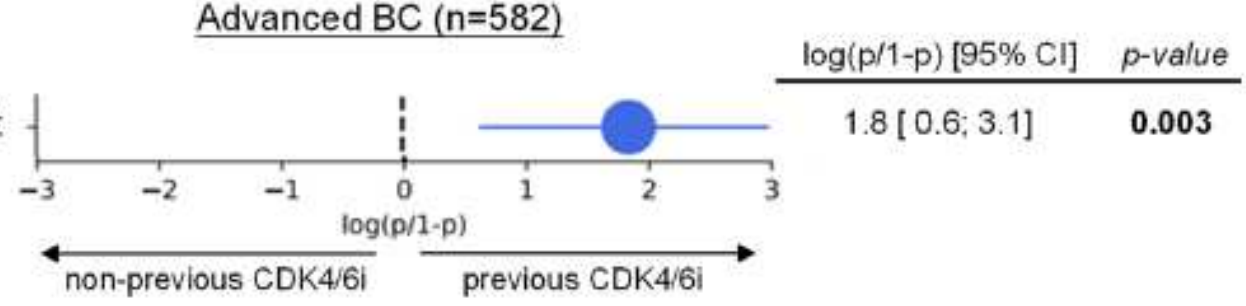

\section{Figure 6}

Acquisition of subclonal RB1 mutations in tumors with underlying RB1 heterozygous loss as mechanism of CDK4/6i acquired resistance in BC PDXs. A) On the top, copy number and mutation status of CDKN2A, RB1, CCNE1 and CCND1 in tumors derived from PDX244, including an untreated sensitive tumor (2R) or tumors with acquired resistance to ribociclib (15L to 19R). Deep-Del, CN <-1; Shallow-Del, $-1 \leq \mathrm{CN}<-0.4$; Unaltered, $\mathrm{CN} \geq-0.4$. Hashtags indicate tumors that acquired deleterious mutations in RB1. On the 
bottom, representative pictures showing $\mathrm{IHC}$ staining of $\mathrm{p} 16, \mathrm{pRb}$, cyclin $\mathrm{E} 1$ and cyclin $\mathrm{D} 1$ from the indicated tumors (bottom). Dashed-red lines mark off areas with different protein staining intensity and protein scoring is indicated. For 19R there was no FFPE tumor available. Magnification 40x. B) IHC staining of $\mathrm{p} 16$ and $\mathrm{pRb}$ in representative FFPE sections from PDX476.1 and PDX476.2. Protein scoring is provided. Magnification 40x. C) Clinical outcome expressed in months for the indicated clinical endpoint in patients with ER+/HER2-, RB1 unaltered tumors vs. those harboring tumors with RB1 heterozygous loss. Data was extracted using the cBioportal (www.cBioportal.org). OS, overall survival; DFS, disease free survival; DOF, days of treatment. D) Association between RB1 double hit alterations (concomitant mutation and deletion) and prior exposure to CDK4/6 inhibitors in metastatic BC patients. The horizontal segment represents the $95 \%$ confidence intervals of the logit value for each test. The size of the circle is proportional to the negative logarithms of the logit p-value. 

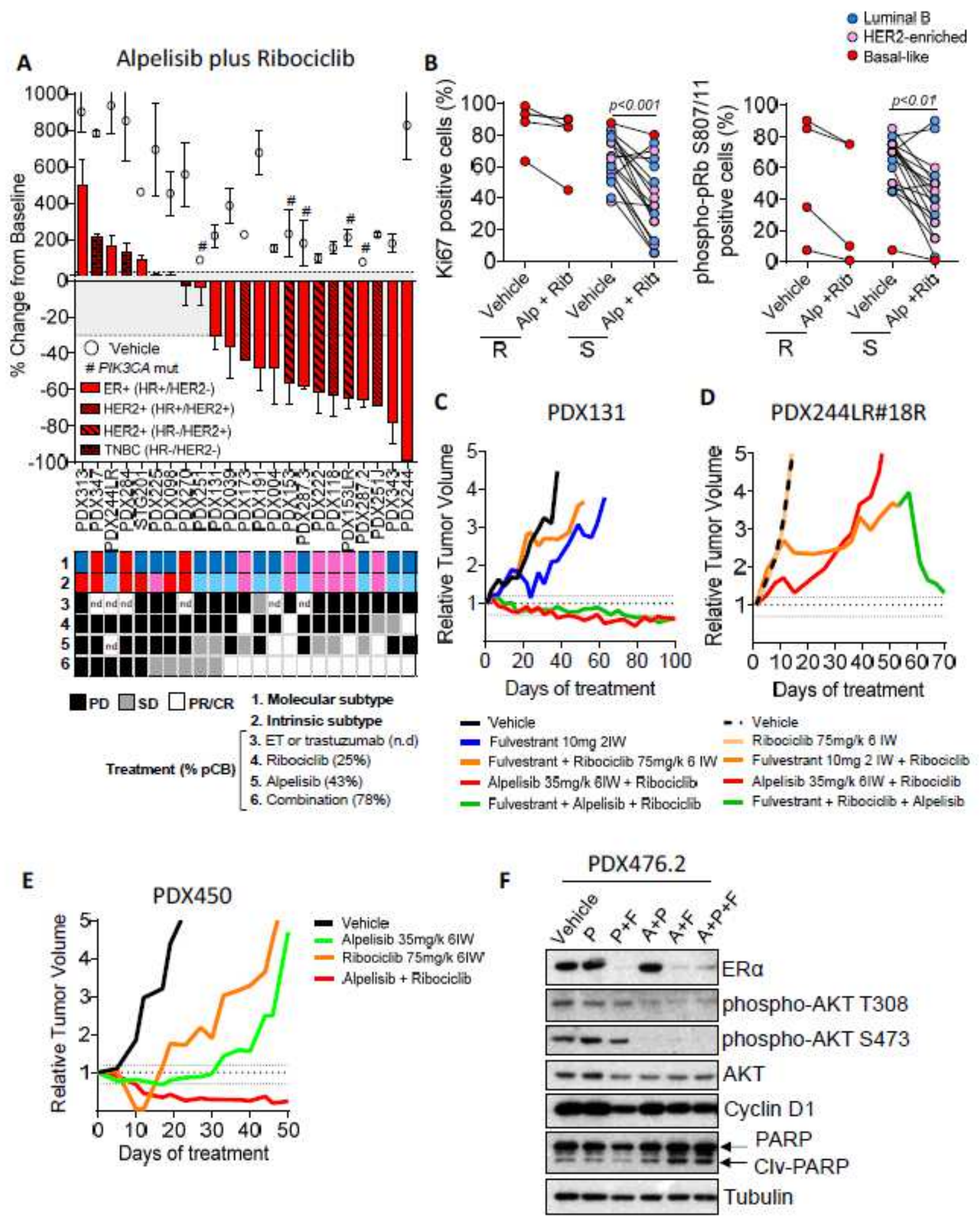

\section{Figure 7}

PI3K inhibition sensitizes non-basal like BC PDX to CDK4/6i. A) Waterfall plot showing the growth of 23 PDX treated with ribociclib $75 \mathrm{mg} / \mathrm{kg}$ plus alpelisib $35 \mathrm{mg} / \mathrm{kg}$ (bars) and vehicle (circles). The percentage change from the initial volume is shown at day 35 of treatment. Dashed lines indicate the range of PD (>20\%), SD (20\% to -30\%) and PR/CR (<-30\%). The molecular subtypes are indicated. Hashtags indicate models harboring mutations in PIK3CA. Data represent mean and error bars \pm SEM. Boxes underneath 
show the molecular and intrinsic tumor's subtypes as well as their responses to the indicated treatments. The preclinical benefit to each drug is indicated as percentage in brackets. n.d, not determined. B) IHC analysis of Ki67 (left graph) and phospho-pRb S807/811 (right graph) in vehicle and alpelisib plus ribociclib treated PDXs in relationship to the PDXs response to alpelisib plus ribociclib. For illustration purposes, the mean value of each PDX was plotted; however, for the statistical analysis all technical replicates were used. p-values are based on Mann-Whitney $U$ test. Different colors represent the tumor's intrinsic subtype. R, resistant; S, sensitive; Alp, alpelisib; Rib, ribociclib. C), D) and E) Relative tumor growth of the ribociclib-resistant PDX131, PDX244LR\#18R and PDX450 after treatment with the indicated drug(s) for the indicated period of time. Dashed lines indicate the range of PD (>1.2), SD (1.2 to -0.7) and PR/CR (<-0.7). F) Immunoblot of indicated the proteins in PDC476.2 treated with vehicle or $500 \mathrm{nM}$ palbociclib as single-agent or combined with $100 \mathrm{nM}$ fulvestrant and/or $2.5 \mu \mathrm{M}$ alpelisib in ex vivo cultures for 48 hours.

\section{Supplementary Files}

This is a list of supplementary files associated with this preprint. Click to download.

- Palafoxetal.Supplementarymaterial.pdf 